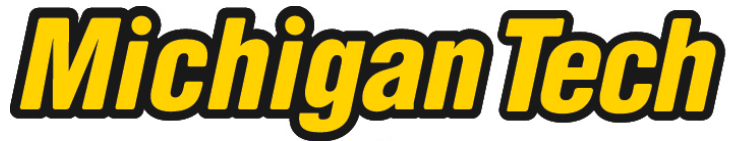 \\ Michigan Technological University Create the Future Digital Commons @ Michigan Tech
}

\section{Bislug flow in circular and noncircular channels and the role of interface stretching on energy dissipation}

Joseph E. Hernandez

Michigan Technological University

Follow this and additional works at: https://digitalcommons.mtu.edu/etds

Part of the Mechanical Engineering Commons

Copyright 2008 Joseph E. Hernandez

\section{Recommended Citation}

Hernandez, Joseph E., "Bislug flow in circular and noncircular channels and the role of interface stretching on energy dissipation", Master's Thesis, Michigan Technological University, 2008.

https://doi.org/10.37099/mtu.dc.etds/372

Follow this and additional works at: https://digitalcommons.mtu.edu/etds

Part of the Mechanical Engineering Commons 
Bislug Flow in Circular and Noncircular Channels and the Role of Interface Stretching on Energy Dissipation

\title{
By
}

Joseph E. Hernandez

\begin{abstract}
A THESIS
Submitted in partial fulfillment of the requirements

for the degree of

Master of Science in Mechanical Engineering

MICHIGAN TECHNOLOGICAL UNIVERSITY

2008
\end{abstract}

Copyright @ Joseph E. Hernandez 2008 
This thesis, "Bislug Flow in Circular and Noncircular Channels and the Role of Interface Stretching on Energy Dissipation" is hereby approved in partial fulfillment of the requirements of the Degree of Master of Science in Mechanical Engineering.

Department of Mechanical Engineering - Engineering Mechanics

Advisor:

Dr. Jeffrey S. Allen

Committee Member:

Dr. Dennis DeSheng Meng

Committee Member:

Dr. Ryan J. Gilbert

Department Chair:

Professor William W. Predebon

Date: 


\section{Abstract}

The area of microfluidics has increased in popularity with such fields as MEMS, microreactors, microscaleheat exchangers, etc. A comprehensive understanding of dissipation mechanisms for fluid flow in microchannels is required to accurately predict the behavior in these small systems. Tests were performed using a constant pressure potential created by two immiscible fluids juxtaposed in a microchannel. This study focused on the flow and dissipation mechanisms in round and square microchannels. There are four major dissipation mechanisms in slug flow; wall shear, dissipation at the contact line, menisci interaction and the stretching of the interface. A force balance between the internal driving potential, viscous drag and interface stretching was used to develop a model for the prediction of the velocity of a bislug in a microchan-

nel. Interface stretching is a dissipation mechanism that has been included due to the unique system properties and becomes increasingly more important as the bislug decreases in length. 


\section{Acknowledgement}

Thanks to NASA for providing the funding for this research. A special thanks to my wife and children and my family for the support, my advisor Jeff Allen for the wisdom, MTU for providing the opportunity, and Cyberia Café for letting me buy a coffee and work all day. 


\section{Nomenclature}

\begin{tabular}{|c|c|c|}
\hline $\mathrm{g}$ & Gravity & $m s^{-2}$ \\
\hline $\mathrm{L}$ & Length & $m$ \\
\hline $\mathrm{U}$ & Velocity & $m s^{-1}$ \\
\hline & \multicolumn{2}{|l|}{ Greek } \\
\hline$\sigma$ & Surface Tension & $N m^{-1}$ \\
\hline$\theta$ & Contact Angle & degrees \\
\hline$\mu$ & Viscosity & $\mathrm{kgm}^{-1} \mathrm{~s}^{-1}$ \\
\hline \multicolumn{3}{|c|}{ Non-dimensional Numbers } \\
\hline Bo & Bond Number & $\Delta \rho g l^{2} \sigma^{-1}$ \\
\hline $\mathrm{Ca}$ & Capillary Number & $\mu U \sigma^{-1}$ \\
\hline
\end{tabular}




\section{Contents}

Abstract

Acknowledgement $\quad$ iv

$\begin{array}{lll}\text { Nomenclature } & \text { v }\end{array}$

Table of Contents vi vi vi vis

List of Figures $\quad$ X

List of Tables $\quad$ xi

1 Introduction 1

1.1 Bislug Definition . . . . . . . . . . . . . . . . . . . 1

1.2 Motivation . . . . . . . . . . . . . . . . 2

2 Background 3

2.1 Capillary Systems . . . . . . . . . . . . . . . . . . . . . . . . 3

2.2 Spontaneous Flow . . . . . . . . . . . . . . . . . . . . 6

2.3 Coating Flows . . . . . . . . . . . . . . . . . . . . 7

2.4 Corners . . . . . . . . . . . . . . . . . . 7

3 Experimental Setup $\quad 10$

4 Numerical Modeling $\quad 14$

4.1 Velocity Profiles . . . . . . . . . . . . . . . . . . 14

4.2 Force Balance . . . . . . . . . . . . . . . . . . . 24

5 Results and Discussion $\quad 29$

5.1 Round Microchannels . . . . . . . . . . . . . . . . . . . . . 29

5.2 Square Microchannels . . . . . . . . . . . . . . . . . . . 32 
6 Force Terms $\quad 40$

6.1 Hydrodynamic Interaction . . . . . . . . . . . . . . . . . . 40

6.2 Contact Line . . . . . . . . . . . . . . . . . . . . 40

6.3 Non-Poiseuille flow . . . . . . . . . . . . . . . . . . . . . . . 41

6.4 Corner Flow . . . . . . . . . . . . . . . . . . . . . 41

$\begin{array}{lll}7 & \text { Conclusion } & 43\end{array}$

$\begin{array}{ll}\text { Bibliography } & 45\end{array}$

A Confocal Imaging of Corner Flow $\quad 46$

B Round Derivation $\quad 48$

B.1 Derivation of Force Terms . . . . . . . . . . . . . . . 48

C Square Derivation $\quad 55$

C.1 Derivation of Force Terms . . . . . . . . . . . . . . . 55

C.2 Drag . . . . . . . . . . . . . . . 57

C.3 Force Balance . . . . . . . . . . . . . . . . . . . . . 62

D Matlab Code $\quad 64$

D.1 Graphing ..................... 64

D.2 Derivation . . . . . . . . . . . . . . . . . . . 78 


\section{List of Figures}

1.1 a) Bislug in a round microchannel, the dyed liquid is ethylene glycol and the non-dyed fluid is silicone oil. b) Schematic of a bislug. . . . . 1

1.2 Schematic of a low-gravity micropump . . . . . . . . . . . . 2

2.1 A wetting fluid rises in a capillary while a non-wetting fluid will recede 3

2.2 Configuration of a liquid drop on a solid surrounded by a gas, the intersection of the three materials create the contact line. . . . . . . 4

2.3 A wetting system has a contact angle $<90^{\circ}$, water on clean glass, and a non-wetting system has a contact angle $>90^{\circ}$, water on wax coated glass. . . . . . . . . . . . . . . . . . .

2.4 Spontaneous flow induced in a microchannel from a pressure imbalanced caused by a (a) difference in surface tension, (b)change in channel dimensions, and/or (c) change in contact angle. . . . . . . . . . 6

2.5 Schematic of a bislug during flow, both fluids $\mathrm{A}$ and $\mathrm{B}$ leave a thin film behind. .......................... 8

2.6 Coating profiles of liquid films in different channel geometries. . . . . 9

2.7 Spontaneous wicking of a fluid into a corner satisfying the Concus-Finn Criteria. ............................. 9

2.8 The schematic of a wedge with spontaneous corner wicking. . . . . . 9

3.1 Experimental setup. . . . . . . . . . . . . . . . . . 11

3.2 Bislug test fixture. . . . . . . . . . . . . . . . . . . . 11

4.1 Assumed flow profiles of the core and film used in the derivation of the models. . . . . . . . . . . . . . . . . . . .

4.2 Flow profiles for the dry round channels, the profiles are for the bounding $\mathrm{Ca}$ numbers for the data. Left (a), is calculated using the low $\mathrm{Ca}$ number, 4E-5 and right (b), uses high Ca number, 2E-4. . . . . . .

4.3 The flow profiles close to the wall for the dry round channels. (a) Left, is for the low $\mathrm{Ca}$ number, $4 \mathrm{E}-5$ and (b) right, is the high $\mathrm{Ca}$ number, 2E-4. 
4.4 Flow profiles for the prewet round channels, the profiles are for the bounding $\mathrm{Ca}$ numbers for the data. Left (a), is for the low $\mathrm{Ca}$ number, 2E-4 and right (b), is the high Ca number, 3E-4. . . . . . . . . .

4.5 The flow profiles close to the wall for the prewet round channels. Left(a), is for the low Ca number, 2E-4 and right(b), is the high $\mathrm{Ca}$ number, 3E-4.

4.6 Geometry for the square channels. The square profile has eight symmetric sections, each of the sections has the profile on the right . . .

4.7 Plots for the low Ca number, 2E-4. (a) Upper left, flow profiles for Core A, no film, (b) upper right, flow profiles with the thin film, Core B and Film A, (c) bottom left, the lines where the profiles were calculate, (d) bottom right, velocity at the transition between the film and the core.

4.8 Plots for the high Ca number, 4E-3. (a)Upper left, flow profiles for Core A, no film, (b) upper right, flow profiles with the thin film, Core B and Film A, (c) bottom left, the lines where the profiles were calculate, (d) bottom right, velocity at the transition between the film and the core.

4.9 The flow profile close to the wall for the square channels. (a) Left, is the low Ca flowrate, 2E-3 and (b) right, is the high Ca number flowrate,

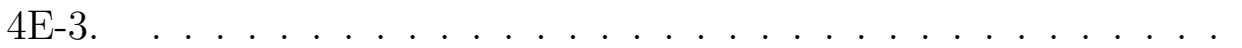

4.10 Drag forces in a round channel, because of the small change in surface area the drag forces overlap. . . . . . . . . . . . . . . .

4.11 The drag in a square channel, the circle and square are the boundaries used for data comparison, low $\mathrm{Ca}$ and high Ca. . . . . . . . . . . 27

5.1 The prewet and non-prewet round channel data. . . . . . . . . . . 30

5.2 Ca numbers for both the model and the Prewet data, the model is based on equation 4.14 using a ratio of $L_{B} / L_{A}=.5,1,2 \ldots$. . . . .

5.3 a) Bislug in a square channel. b) Fluid retention in the corner of a prewet square microchannel . . . . . . . . . . . . . . . . 32

5.4 Graph of Ca for square dry channel . . . . . . . . . . . . . . . 33

5.5 Ca number of ethylene glycol based on the front meniscus and the ratio of the slug length to the radius. . . . . . . . . . . . . . .

5.6 Ca number of ethylene glycol based on the rear meniscus and the ratio of the slug length to the radius. . . . . . . . . . . . .

5.7 Images used to estimated the film thickness on the walls of a square microchannel. (a) Used for scaling images. (b) and (c) Used for measuring the amount of fluid retained in the corner and the width of the flat region of the prewet film.

5.8 The different energy dissipation zones present in bislug system based on the data collected from the front meniscus. . . . . . . . . . . .

5.9 The different energy dissipation zones present in bislug system based on the data collected from the rear meniscus. . . . . . . . . . . 
5.10 Comparison of the experimental data to the developed model with and without stretch. (a)left, data from the front of the meniscus (b)right, the same data plotted on a $\log -\log$ graph. . . . . . . . . . . . .

5.11 Comparison of the experimental data to the developed model with and without stretch. (a)left, data from the rear of the meniscus (b)right, the same data plotted on a $\log -\log$ graph. . . . . . . . . . . .

6.1 Recirculation caused by faster centerline velocity relative to the menisci, faster velocity is required to satisfy the conservation of mass for the

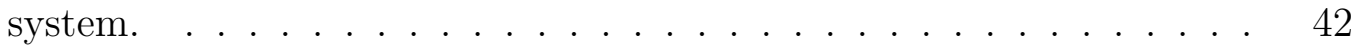

A.1 Corner flow captured using a LSCM. . . . . . . . . . . . . . 47 


\section{List of Tables}

3.1 Test parameters for ethylene glycol and silicone oil in circular and noncircular microchannels, the round channel has a radius of 0.469 $\mathrm{mm}$ and the square has a hydraulic radius of $.5 \mathrm{~mm} . \quad \ldots \ldots \ldots$

4.1 Parameters for Round graphs . . . . . . . . . . . . . . 15

4.2 Parameters for Square graphs . . . . . . . . . . . . . . 20

4.3 Coefficients for $F_{d r a g}$ in the square channels $\ldots \ldots \ldots \ldots 27$

C.1 Coefficients for $F_{d r a g}$ in the square channels $\ldots \ldots \ldots 63$ 


\section{Chapter 1}

\section{Introduction}

Research for this paper focused on the behavior of a system consisting of two immiscible fluids in a microchannel. When two immiscible fluids are in contact in a capillary, spontaneous flow may occur[4]. The original goal of the project was testing the feasibility of a micropump using the spontaneous capillary motion of the two immiscible fluids. After the micropump was found to be feasible, the scope of the research expanded to include the study of dissipation mechanism in microchannels.

\subsection{Bislug Definition}

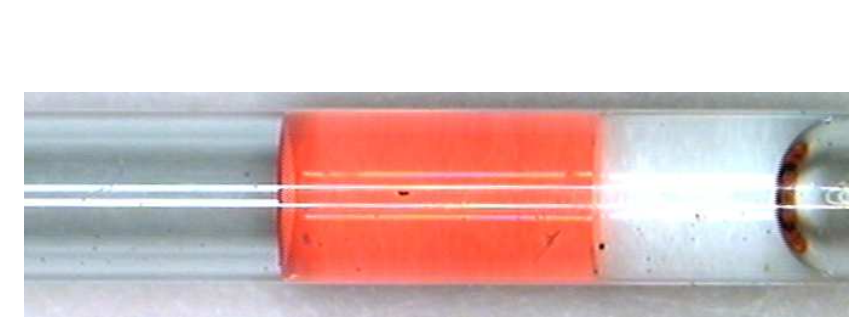

(a)

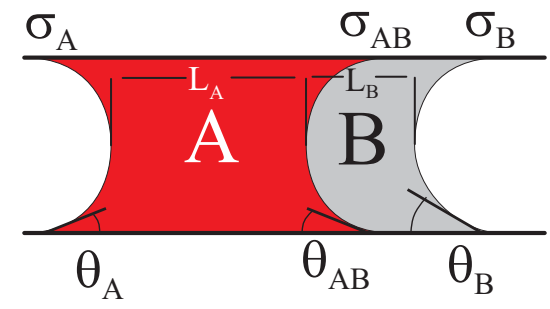

(b)

Figure 1.1. a) Bislug in a round microchannel, the dyed liquid is ethylene glycol and the non-dyed fluid is silicone oil. b) Schematic of a bislug.

A bislug is defined as two immiscible liquids in contact forming a slug in a microchannel. Figure 1.1(a) is an image of a bislug in a round channel, the darker fluid is ethylene glycol and the light-colored fluid is silicone oil. The subscripts used in the discussion are based on the definitions from Figure 1.1(b), the front liquid will be labeled "A" and the trailing liquid "B". Slug lengths are measured at the center of one meniscus to the other. Properties at the different interfaces are labeled "A" for air-liquid A, "B" for air-liquid B, and "AB" for the liquid-liquid interface. A pressure potential is created over the menisci due to a change in the surface tension, a description of the mechanisms for spontaneous flow can be found in the Background, 


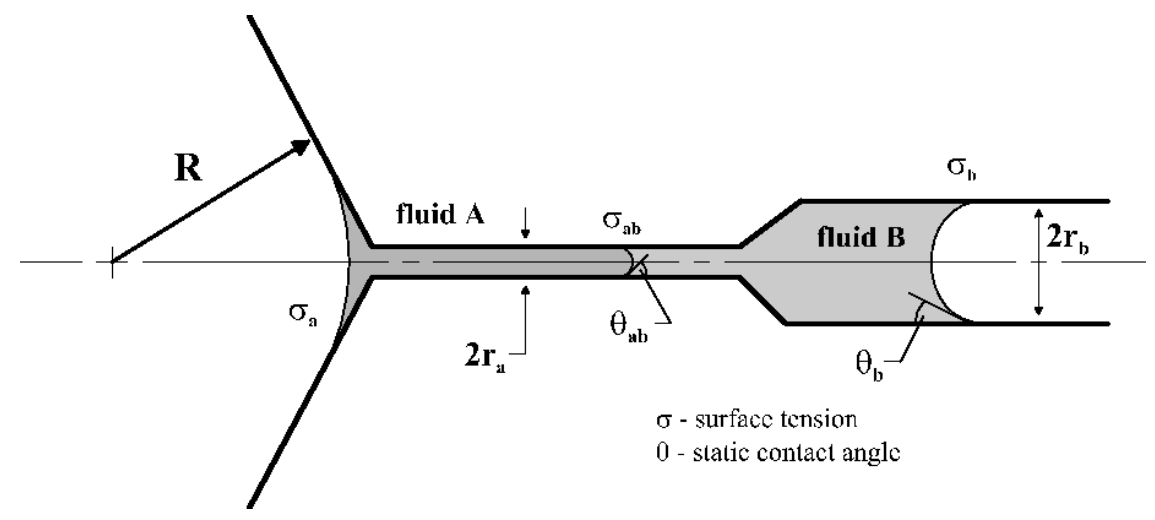

Figure 1.2. Schematic of a low-gravity micropump

Chapter 2. The bislug flows in the direction from right to left relative to Figures 1.1(a) and 1.1(b).

\subsection{Motivation}

\subsubsection{Passive Micropump}

The original goal of the research was a feasibility study for the design of a low-gravity micropump for maintaining a surface of constant curvature. The pressure potential from the bislug can be balanced by manipulating the geometry of the channel, see Figure 1.2. A passive pump is created by the tapered endpiece of the channel. The leading slug will change its radius of curvature until the pressure potential is balanced and the flow is stopped. If fluid is drained from the cone the radius of curvature is changed and the bislug will move to the balanced position. It has been observed from experiments that the response in a round channel may be too slow for a proper fluid pump. A square channel, because of the geometry, has a faster response time resulting in a feasible micropump.

\subsubsection{Energy Dissipation}

While the motivation for study of the bislug pump originated with the need for a passive pump, the opportunity for a more fundamental investigation of microchannel fluid flow was realized. The constant pressure potential created from the bislug may provide an ideal way for studying dissipation mechanisms in a microchannel. From test to test the pressure potential will remain constant regardless of the slug lengths as long as the channel and fluid properties remain constant.

There are four main dissipation mechanisms in a microchannel; viscous shear, contact line dissipation, menisci interaction and the energy associated with the stretching of an interface. A bislug velocity model based on the viscous drag and the interface stretching has been developed and compared to experimental data in both round and square microchannels. 


\section{Chapter 2}

\section{Background}

\subsection{Capillary Systems}

Bislugs can spontaneously flow in capillary scale channels where the gravitational affect on the interface shape is minimized. A common example of capillarity is a capillary tube placed in a container of fluid. Because of a pressure imbalance at the meniscus the fluid will rise in the channel if it is wetting, or recede if the fluid is non-wetting, Figure 2.1.

To determine whether the system is in the capillary range, where gravitational affects on the interface are minimal, the Bond number is used. The Bond number is the ratio of gravitational to surface tension forces.

$$
B o \equiv \frac{\Delta \rho g L^{2}}{\sigma}
$$

where $\Delta \rho$ is the density difference accross the meniscus, $\sigma$ is the surface tension of the meniscus, and $\mathrm{g}$ is the local gravitational acceleration. When the $B o \ll 1$ the affects of gravity may be neglected when describing the interfacial shape.

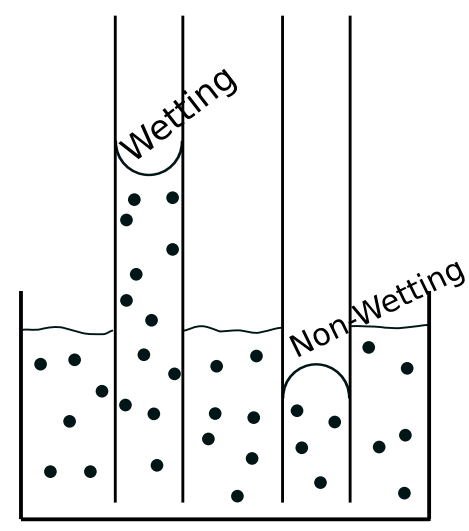

Figure 2.1. A wetting fluid rises in a capillary while a non-wetting fluid will recede 


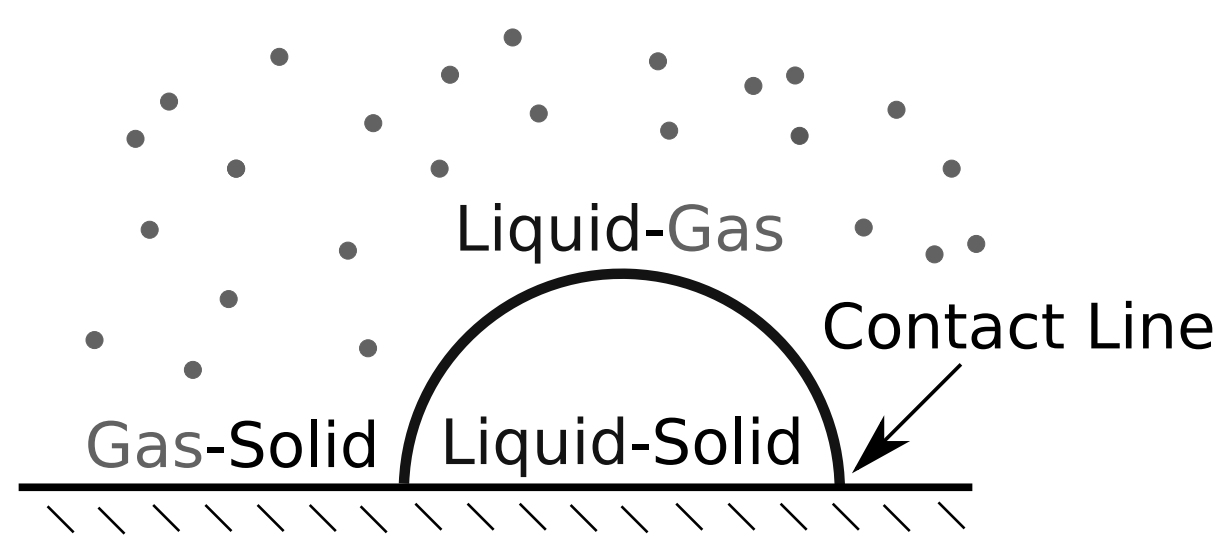

Figure 2.2. Configuration of a liquid drop on a solid surrounded by a gas, the intersection of the three materials create the contact line.

A useful variation of the Bo number is the capillary length or Laplace constant, $L_{c}$. The Laplace constant is found by setting the Bo number equal to one and solving for the characteristic length,

$$
L_{c}=\sqrt{\frac{\sigma}{\rho g}}
$$

. This length is the transition between surface tension and gravity dominated systems.

Though the interfacial shape of the system may not be affected by gravity when the Bo $\ll 1$, gravity is still acting on the buld fluid. A common misconception is the bulk system is not affected by gravity but a couple of examples will demonstrate otherwise. First, a drop from a cloud or a faucet can easily have a $B o \ll 1$ but it will still fall towards the earth. Second, a sponge, with pores in the capillary range, will spontaneously draw water in. Yet, in an unsaturated sponge the water will not be distributed uniformly. Rather, the water will accumulate in the lower portion of the sponge.

Interfacial shapes in a capillary system are dominated by the surface tension of the fluid(s). Surface tension, $\sigma$, is the molecular cohesion force acting at the interface between two fluids and is unique for a given fluid pair. The interfacial shape is determined by surface tension, a surface energy, which tries to minimize the overall surface area, this produces a spherical shape in simple configurations, Figure 2.2. The shape of a fluid on a solid surface is a function of the interaction at the contact line. For simplicity, a drop of liquid will be placed on a solid with a gas as the surrounding fluid, Figure 2.2. The contact line is the intersection of the three materials, gas, liquid and solid. The balanced interfacial energies, gas-liquid, gas-solid, liquid-gas, determine the final shape of the liquid. At the contact line the interaction is quantified using the contact angle. The contact angle, $\theta$, is measured from the solid to the tangent at the surface of the liquid at the contact line, Figure 2.3. A liquid is considered wetting if $\theta<90^{\circ}$ and non-wetting if $\theta>90^{\circ}$. 


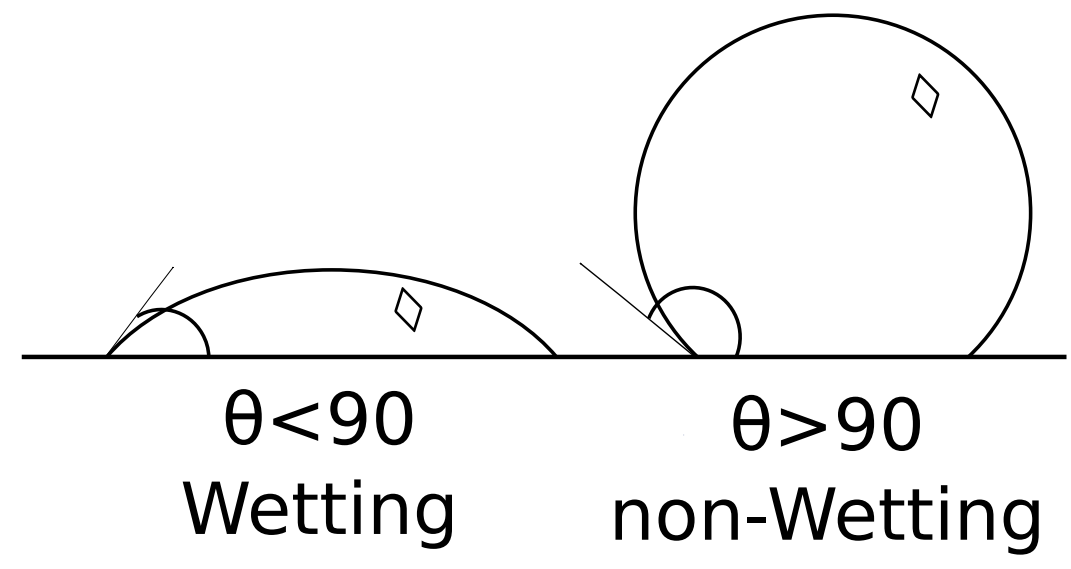

Figure 2.3. A wetting system has a contact angle $<90^{\circ}$, water on clean glass, and a non-wetting system has a contact angle $>90^{\circ}$, water on wax coated glass.

A pressure differential is caused by the curvature of the fluid interface and can be calculated using the Laplace-Young equation.

$$
P_{\text {liquid }}-P_{\text {gas }}=\Delta p=\sigma k
$$

$k=1 / r_{1}+1 / r_{2}$ Where $r_{1}$ and $r_{2}$ are the principle radii of curvature. For a round channel this reduces to $\Delta P=2 \sigma / R$, where $R=a / \cos \theta, a$ is the radius of the channel and $\theta$ is the contact angle. For a perfectly wetting fluid, $\theta=0, \Delta P=2 \sigma / R$. Material and geometric properties of a channel which affect the pressure drop are:

1. The radius of curvature changes with contact angle and radius of the channel

2. Fluids with differing surface tensions will have different pressure drops in the same channel

The pressure drop from the curvature of a fluid is the reason why a fluid will wick into a capillary tube or napkin, Figure 2.1. The radius of curvature in the capillary, $R=a / \cos \theta$, is smaller than the curvature on the surface, $R \approx \infty$. This produces a net pressure drop and the fluid is pushed in the capillary until the capillary force is balanced by gravitational. Washburn (1921) formulated the first widely reconized predictive equation for the rise time in both a horizontal and vertical oriented channel.

Velocites in microchannels can be represented in a non-dimensional form called the Capillary number,

$$
C a \equiv \frac{\mu V}{\sigma}
$$

, where $\mu$ is the viscosity of the fluid, $V$ is the velocity of the fluid and $\sigma$ is the surface tension. The Capillary number is the ratio of viscous forces with surface tension. The Ca number is used extensively in the non-dimensional form of the models developed for the bislug systems. 

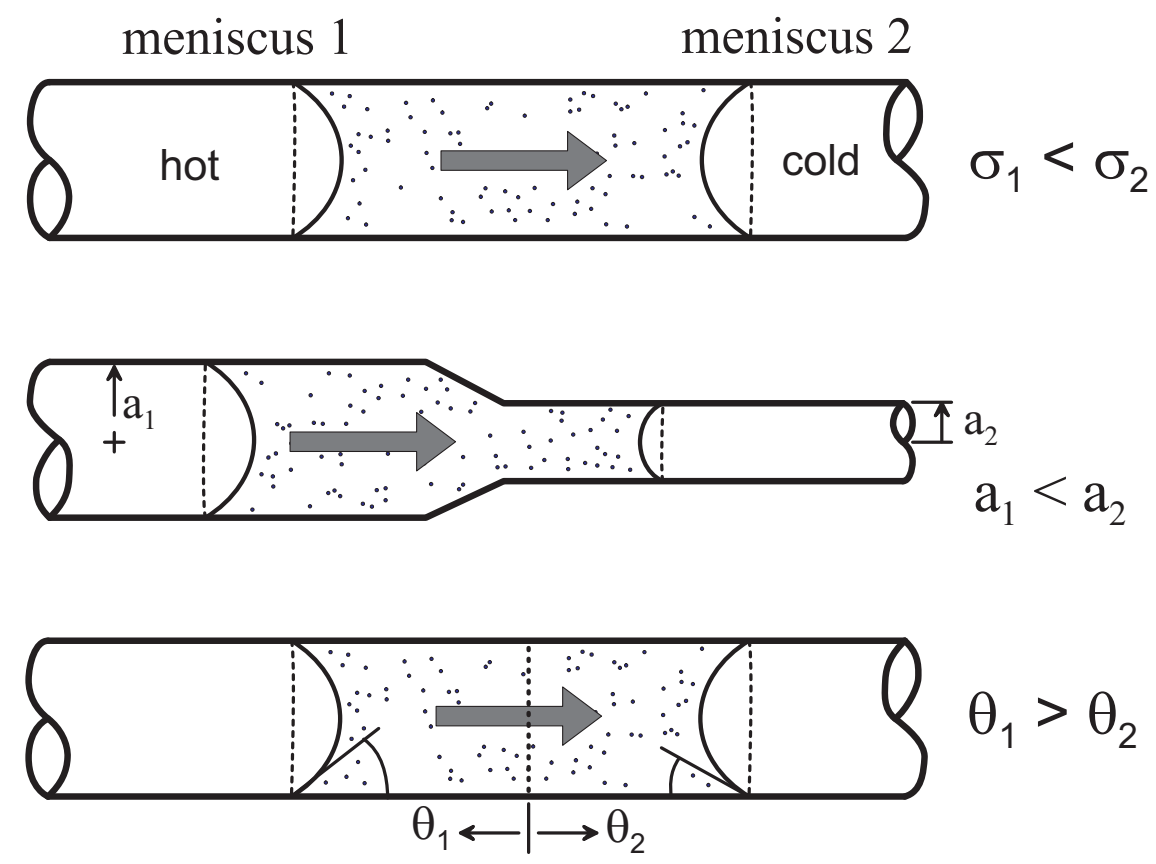

Figure 2.4. Spontaneous flow induced in a microchannel from a pressure imbalanced caused by a (a) difference in surface tension, (b)change in channel dimensions, and/or (c) change in contact angle.

\subsection{Spontaneous Flow}

Spontaneous flow in microchannels occurs through a pressure imbalance accross the menisci of a slug. Figure 2.4 shows three different configurations that can result in spontaneous motion; each of which is a manipulation of the Laplace-Young equation 2.3. A difference in surface tension across a slug will cause flow in the direction of the fluid with the highest surface tension 2.4(a). A change in channel geometry, such as an increase or decrease in radius, will change the radius of curvature in the system, which results in flow towards the smaller radius of curvature 2.4(b). Changing the contact angle also changes the radius of curvature, producing flow towards the smaller contact angle 2.4(c).

When two immiscible fluids are in contact, a net pressure drop is produced from the difference in surface tension across the three menisci, resulting in spontaneous motion. The motion will be towards the fluid with the highest surface tension. This assumes that the radius of the channel and the contact angles of the two fluids are equal. This phenomenom was first studied in great detail by Bico and Quéré(2002). Their work focused on bislug flow in round channels using both miscible and immiscible fluid pairs. 


\subsection{Coating Flows}

When a slug of liquid travel down a channel a thin film of fluid is left on the walls. This can be seen with a classic example of a bubble displacing a fluid in a channel [17]. As the bubble moves forward the fluid is pushed out of the channel but leaves behind a thin film of fluid. This film acts a lubricating layer for the bubble to travel on, producing bubbles that travel faster than the liquid film[10].

Previous research involving coating flows has been directed towards oil recovery enhancement. Water forms a lubricating layer that the oil can travel over, since oil and water are immiscible they can be easily seperated. A comprehensive comparison of data to old and new theory can be found in Bai(1992). Essentially, based on flow rates of the two fluids, different instabilities are formed in the pipes. This is of extreme importance as the amount of energy required changes for the different flow regimes[2]. Any increase in energy requirements to push the fluids would mean a large increase in expenditure for oil recovery, due to the large volumes of water used[1]. An empirical formula for the film thickness, h, was developed by Bretherton(1961),

$$
\frac{h}{R}=.634 C a^{(2 / 3)}
$$

where $\mathrm{R}$ is the capillary radius. Thus, the film thickness can be estimated by knowing the $\mathrm{Ca}$ number of the system and radius of the channel.

A constant pressure drop is created during bislug flow due to the interfacial shape created by the constancy of the surface tension. The pressure potential in a bislug acts only over the menisci. The film, which is assumed to be isolated from the menisci, experiences a flow potential from a moving interface. This differs from common annulus flow experiments where a pressure drop is supplied over the entire radius of the channel. The film experiences an external pressure potential which drives the fluid in the film producing a different flow profile than that of the film in a bislug. As a bislug travels down the channel it leaves a film of fluid A which fluid B travels over, Figure 2.5. The film of fluid A is subjected to drag from core B and the film is dragged along similar to Couette flow. Fluid B also deposits a thin film over the film of fluid A. Solutions for the flowrate for both cases can be found in Middleman(1995).

\subsection{Corners}

A uniform film thickness is deposited on the walls of a round microchannel when coated with wetting liquid. A square microchannel exhibits a different form of coating. As a fluid slug traverses a square microchannel it leaves a thin film on the walls similar to the round channels, but it may also deposit a thicker film in the corners, Figure 2.6. Whether or not the fluid is retained in the corners is dependent on the Concus-Finn criteria [8]. The criteria for corner wicking is a function of the contact angle of the fluid and the surface, $\theta$, and the half angle of the corner, $\alpha$, Figure 2.7.

$$
\theta \leq \frac{\pi}{2}-\alpha
$$




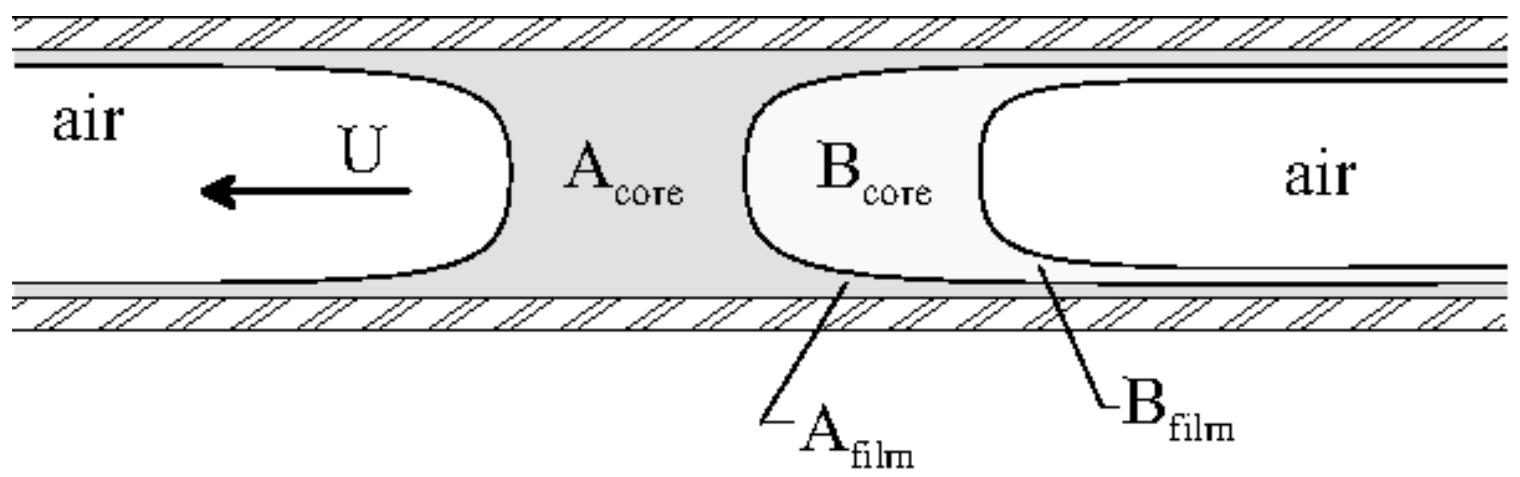

Figure 2.5. Schematic of a bislug during flow, both fluids A and B leave a thin film behind.

Any channel with corners can have fluid spontaneously wick into the corners if it satisfies the Concus-Finn criteria. The spontaneous flow of the fluid is induced by a difference in curvature. This curvature difference causes a net pressure imbalance in the same manner as Figure 2.4(b). Figure 2.8 represents fluid in a wedge, the wedge is one of the four corners of a square channel, the radius of curvature reduces from left to right. The reduction in curvature can theoretically occur to infinity, resulting in an infinite flow potential. The smallest radius of curvature is limited by either the scale required to continue coating is smaller than the thickness of a molecule of the fluid or the more likely, a channel defect. The channel defect can be a result of contamination in the corner which provides a location to pin and stop the flow or a manufacturing defect, such as rounded corners. The radius of the rounded corner is the smallest radius the fluid can have, limiting the overall distance for the spontaneous flow. The "finger", fluid displaced from the meniscus, which is produced from the corner flow can be characterized by $\mathrm{H}$ [22]. $\mathrm{H}$ is the length of the displaced from fluid from the meniscus to the end of the fluid finger. This can be thought of as the transition between the spherical mensiscus and the fluid retained in the corners. Based on modeling from Weislogel(1996), the value of the transition can be calculated for a given channel geometry.

For the test setup both fluids are perfectly wetting to the glass and the square microchannels have corners with half angles of $45^{\circ}$. Since both liquids are perfectly wetting to glass, $\theta<45^{\circ}$, these will spontaneously wick into the corners of the square channels. Fluids with contact angles between $45^{\circ}$ and $135^{\circ}$ are partially wetting in square microchannels; that is, the fluid behaves like it would in a round channel. 


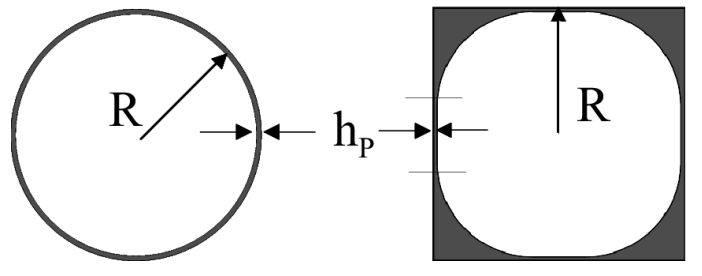

Figure 2.6. Coating profiles of liquid films in different channel geometries.

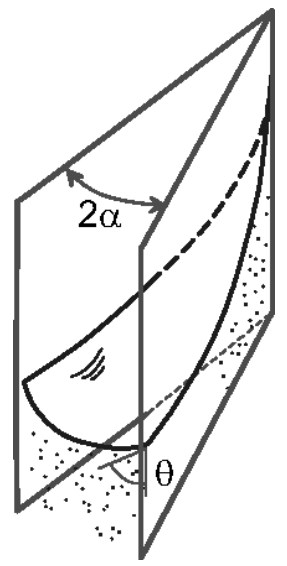

Figure 2.7. Spontaneous wicking of a fluid into a corner satisfying the ConcusFinn Criteria.

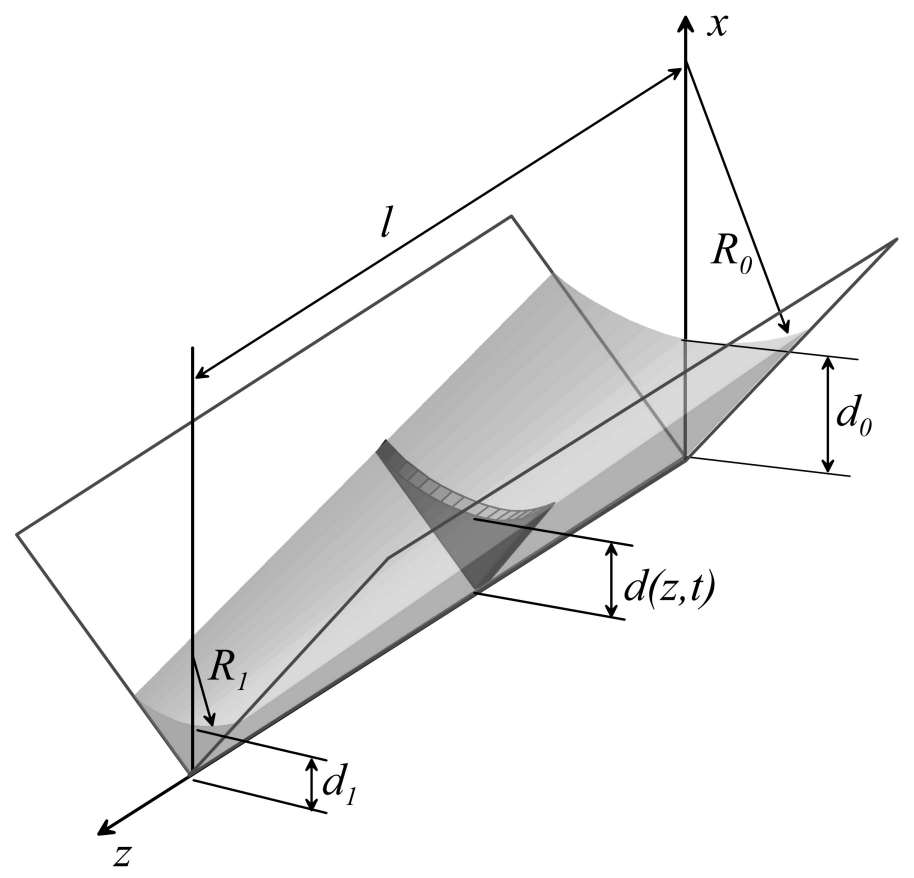

Figure 2.8. The schematic of a wedge with spontaneous corner wicking. 


\section{Chapter 3}

\section{Experimental Setup}

Video of the bislug was recorded using standard image capturing techniques. A Panasonic GP-KS152 color CCD camera was attached to an optics port on a Nikon SMZ1500 microscope. The microscope was attached to boom stand, with six degrees of freedom for coarse microscope adjustment. The boom stand was setup on a $\mathrm{x}-\mathrm{y}$ translation stand (Velmex A25) which allows for fine adjustment in the $\mathrm{x}-\mathrm{y}$ directions and the microscope focusing adjusted in the z-direction. A video capture card, Epix model PIXCI SV5, was used to record the image sequences from the camera to a computer (IBM 8307-22U). The Epix board had a working frame rate of approximately 30 frames per second (fps). Image files were saved using a .tiff format to retain pixel information which may be lost using other standard image file formats. Capturing of a crisp interface required a fast shutter speed and proper lighting. The shutter speed was set to 1/1000 of a second. As the shutter speed increases the amount of light needed also increases. The first attempt at lighting used a ring light attached to the microscope lens. This provided enough light but also produced flares where the light crossed the glass channels. To remove the flares diffused light was used, this required a transmitted illumation setup. For the diffused lighting the channel test fixture was made from glass. Microscope covers were glued onto a microscope slip with enough space to set the microchannel into place. Using this setup the aligment of the microchannels could be maintained for all test runs; the microchannels were placed in the same direction and maintained the same focal distance. The image scaling was kept constant between test runs because the magnification was not changed. Images of the experimental setup can be seen in Figures 3.1, 3.2.

Ethylene glycol and silicone oil were used for the immiscible fluid pair [5]. These are the same fluid pair used by Bico [4] in the original bislug experiments. Properties for the fluids were taken from Bico [5] and are listed on Table 3.1. For better contrast, the ethylene glycol was dyed using red food coloring, which is a mixture of coloring, propylene glycol, and water. Only a small amount of coloring was used to minimize the affect on the ethylene glycol properties. The silicone oil was not dyed because the contrast between the ethylene glycol and silicone oil was sufficient for image 


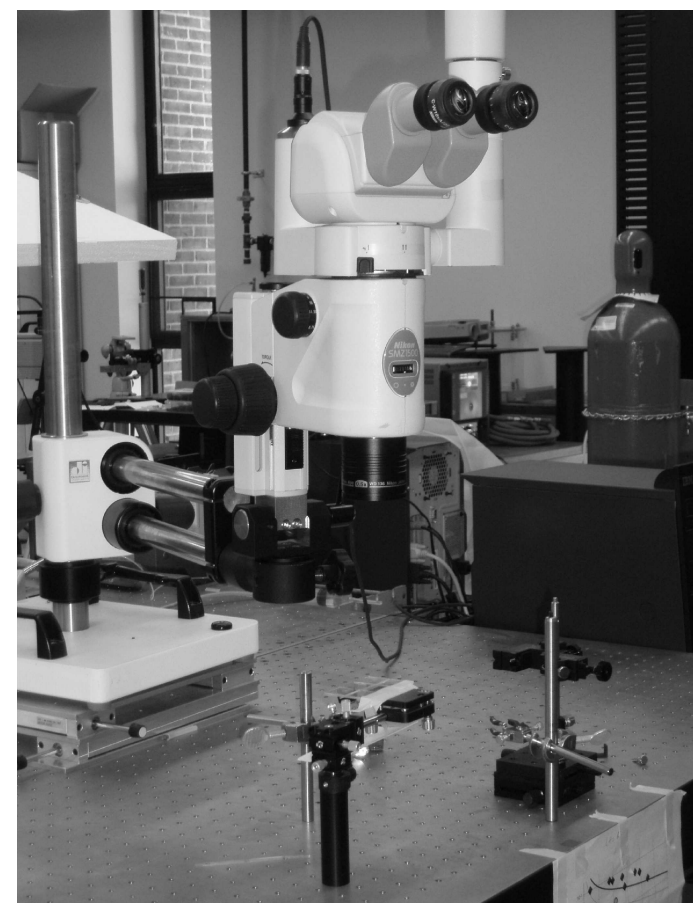

Figure 3.1. Experimental setup.

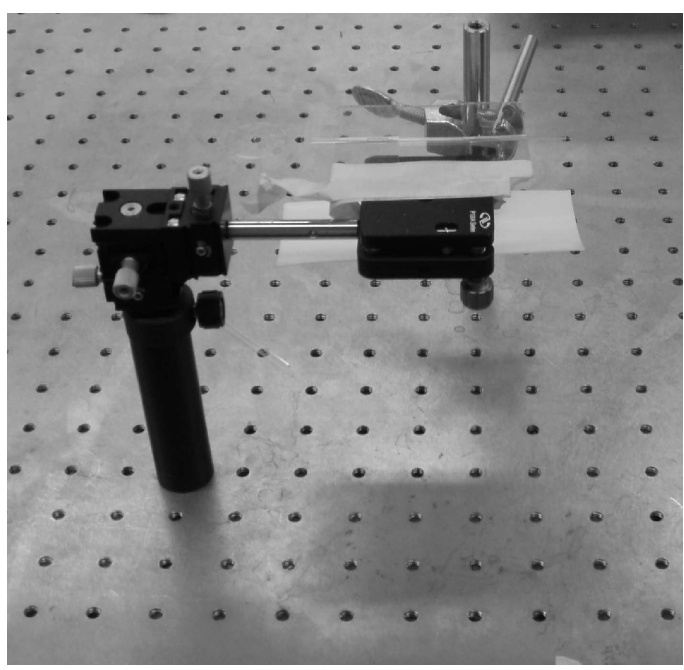

Figure 3.2. Bislug test fixture.

processing.

Round and square micro-channels made from boroscilicate glass were used for the test sections. These channels were chosen to be consistent with previous work and the fluids are perfectly wetting to the glass; therefore the low contact angle satisfies the Concus-Finn criteria for square microchannels. The round microchannels are $40 \mu \mathrm{L}$ yellow band disposable pipets manufactured by Drummond Scientific. The channels are drawn with an average innner diameter of approximately $0.938 \mathrm{~mm}$, which was calculated from knowing the volume and calibrated fill length of the channel. The square channels had an inner side dimensions of $1 \mathrm{~mm}$ square and were manufactured by Vitrocom. Microchannels with hydraulic diameters close to $1 \mathrm{~mm}$ were chosen for optical clarity. As the channel diameters decrease a higher magnification is needed to view the menisci, the higher magnification decreases the viewable working area of the microchannel. A large working area is prefered to increase the amount of time the bislug remains in the field of view. This decreases the amount of error in the data processing. Smaller diameter channels also produce longer slugs than large diameter channels for the same fluid volume which creates difficulties when trying to control slug lengths.

The bislug was created using two different methods. To create the bislug using the first method, a channel was dipped into a vial containing ethylene glycol. Ethylene glycol was drawn into the microchannel by capillary forces. To prewet the channel, the tubes were inverted and gravity pushed the slug of ethylene glycol down the channel; 
thus coating the channel walls with a film of liquid. The slug length of ethylene glycol could be adjusted by wicking some fluid out using a paper towel. The prewet channel was then dipped into the silicone oil allowing capillary forces to draw fluid in to the channel adjacent to the ethylene glycol. Keeping the channel vertical prevented long slugs of silicone oil from forming, the length of silicone oil depended on the length of the ethylene glycol, to increase the length of either slug the channel was tilted to reduce the hydrostatic head. For test runs requiring nonprewet channels, the ethylene glycol slug was inserted into the silicone oil vial after adjusting the slug length. Once the bislug was formed the microchannel was placed in the test fixture.

A second method, for generating bislugs used syringes to accurately control the lengths of the slugs. Thin needles were attached to both push and screw style syringes. The screw style provided tighter control over the volume being inserted. Ethylene glycol could be accurately deposited in the microchannels but prewetting the system was difficult. To prewet, the channels had to removed from the setup, turned vertical and realigned after the prewetting was complete. Longer slugs, when tipped vertical, would drain because the force of gravity was larger than the capillary forces. To make longer slugs, the microchannel was filled with a small slug and inverted to wet the walls. Then microchannel was realligned and the syringe was used to increase the size of the slug. After injection of the ethylene glycol a syringe with silicone oil was used to create the bislug. This procedure, though more precise, was not practical due to the spontaneous nature of the bislug flow. As soon silicone oil was deposited the bislug began to flow. This prevented the deposition of long silicone oil slugs. Bubbles could also be trapped in the system due to the shape of the meniscus. Without gravity pushing the slug down, as in the previous method, the curved meniscus created a gap between the meniscus and the microchannel end. In a square microchannel an ethylene glycol slug would migrate down the channel from its initial position to balance the pressure gradient caused by the corner flow. This did not allow for the silicone oil to be deposited without an air bubble. Different techniques were attempted to prevent the bislug from moving, but each attempt was unsuccessful. This technique proved to be too cumbersome and the first method was used.

Spotlight, an image tracking software developed at the NASA Glenn Research Center by B. Kilmek and T. Wright, was used for measuring the bislug systems [12]. An interface tracking Area-of-Interest(AOI) was setup to track both the front and rear menisci. Lines produced by the NTSC video were smoothed to produce an average interface and a low pass filter was used to create a sharp tracking edge. The software recorded the change of position with time, values for the length and framerate were used for scaling. Spotlight outputs an ASCII file containing the information regarding scaling and the values from the tracking AOI. These text files were imported to Lotus 123, Excel, and MATLAB for data processing. Acceleration and velocity of the bislug were determined by using a least square polynomial fit of the position and time. Errors from the data collection were determined to be \pm 2 pixels for the length measurements 
and \pm 2 fps for the time measurement. The value of 2 pixels is the measurement error for the length of a slug. Measurements in Spotlight are accurate to a pixel in both the $x$ and $y$ directions, the decimal values are from the norm of the components. Video captured using the CCD camera is interlaced, half of the frame updates every 1/2 fps, this value was chosen for the error that can occur between frames.

Inconsistencies in both the prewet and dry data may be attributed to the quality of the microchannel used. The round and square microchannels are manufactured by drawing glass over a mandrel. Imperfections in the process can produce channels with fluctuatations in the radius over the length of channel, these fluctuations will cause a change in the instantaneous velocity. This process also produces square channels with rounded corners, which limits the pressure developed in the corners from the spontaneous fluid flow.

Table 3.1. Test parameters for ethylene glycol and silicone oil in circular and noncircular microchannels, the round channel has a radius of $0.469 \mathrm{~mm}$ and the square has a hydraulic radius of $.5 \mathrm{~mm}$.

\begin{tabular}{r|cc|c} 
parameter & $\begin{array}{c}\text { ethylene } \\
\text { glycol } \\
(\mathrm{A}-\text { air })\end{array}$ & $\begin{array}{c}\text { silicone } \\
\text { oil } \\
(\mathrm{B}-\text { air })\end{array}$ & $(\mathrm{A}-\mathrm{B})$ \\
\hline$\nu[\mathrm{cSt}]$ & 16 & 1 & 150 \\
$\Delta \rho\left[\mathrm{kg} / \mathrm{m}^{3}\right]$ & 1113 & 963 & \\
$\mu[\mathrm{kg} / \mathrm{ms}]$ & 0.0178 & 0.0096 & $18.0 \pm 0.2$ \\
$\sigma[\mathrm{mN} / \mathrm{m}]$ & $47.7 \pm 0.1$ & $20.3 \pm 0.1$ & 3.50 \\
\hline$L_{c}[\mathrm{~mm}]$ & 2.09 & 1.47 & $0.018-0.020$ \\
Bo & $0.050-0.057$ & $0.10-0.12$ &
\end{tabular}




\section{Chapter 4}

\section{Numerical Modeling}

To model the flows in both the round and square channels common flow profiles were assumed. For the core of the slugs, where the menisci pressure potential acts, a classic Hagen-Poiseuille flow profile was assumed. A Couette flow profile was used for the film, which assumes the primary driving potential for the flow is caused by the moving liquid-liquid interface. Boundary conditions of equal velocity and shear were used for the transition from the core to the film. Figure 4.1 shows the profiles used for the core and the film; no slip at the wall, $U_{A B}$ is the velocity at the liquid-liquid interface and $U_{\max }$ is the centerline velocity. The full derivation for the round channels is in Appendix B and the square channel derivation is in Appendix C.

\subsection{Velocity Profiles}

\subsubsection{Round Channels}

As mentioned above, Hagen-Poiseuille flow was used in the core and a Couette profile was used in the film. The boundary conditions used were: no-slip at the wall, a symmetry at the core centerline, and continuity in velocity and shear at the liquid-liquid interface. Solutions for the velocity profiles are presented below, see Appendix B for the full derivation:

$$
\begin{gathered}
u_{\text {Core A }}=U_{\max }\left[1-\left(\frac{r}{R}\right)^{2}\right] \\
u_{\text {Film A }}=U_{A B} \frac{\ln \left(\frac{R}{r}\right)}{\ln \left(\frac{R}{R-h_{A}}\right)} \\
u_{\text {Core B }}=\left(U_{\max }-U_{A B}\right)\left(1-\frac{r^{2}}{\left(R-h_{A}\right)^{2}}\right)+U_{A B}
\end{gathered}
$$

where

$$
U_{A B}=U_{\max }\left[1+\frac{1}{2} \frac{\mu_{A}}{\mu_{B}} \frac{1}{\ln \left(\frac{R}{R-h_{A}}\right)}\right]^{-1}
$$




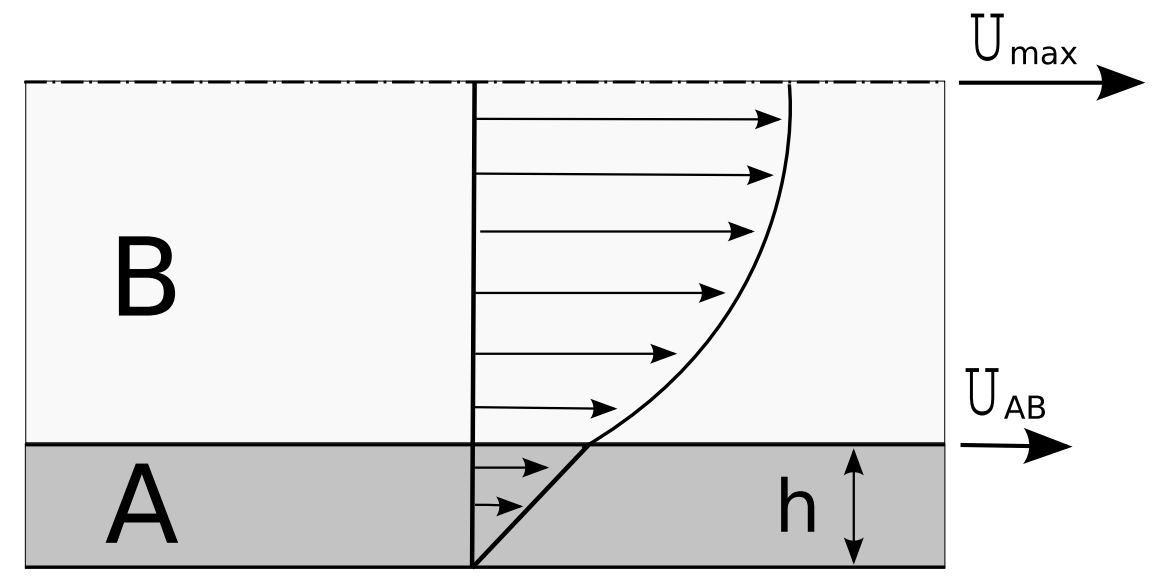

Figure 4.1. Assumed flow profiles of the core and film used in the derivation of the models.

The solution for the velocity in the thin film, equation 4.2, matches the solution from previous derivations of concentric cylinders with the inner cylinder moving [23]. Since the boundary conditions assumed constant velocity and shear at the liquidliquid interface between the film of fluid A and core of fluid B the equation for the transition velocity contains elements of both profiles and introduces a logarithmic term to the Hagen-Poiseuille profile.

For modeling purposes the domain was examined at the upper and lower limits for the Ca number of the experimental data because the film thickness is calculated using equation 2.5. Both the flow for the prewet and dry test runs were modeled using the derived equations.

Table 4.1. Parameters for Round graphs

\begin{tabular}{|c|c|c|c|}
\hline Test Runs & Ca & $U_{\max }(\mathrm{mm} / \mathrm{s})$ & $h_{o}(\mu m)$ \\
\hline Dry Round Low Ca fig 4.2(a) & $4 \mathrm{E}-5$ & 0.1072 & 0.7822 \\
Dry Round High Ca fig 4.2(b) & $2 \mathrm{E}-4$ & 0.5360 & 2.2871 \\
Prewet Round Low Ca fig 4.4(a) & $2 \mathrm{E}-4$ & 0.5360 & 2.2871 \\
Prewet Round High Ca fig 4.4(b) & $3 \mathrm{E}-4$ & 0.8039 & 2.9969 \\
\hline
\end{tabular}

Flow profiles for both the dry and prewet channels are presented in Figures 4.2 and 4.4, respectively. The flow profile for Core A (no film) and Core B/Film A are plotted on the same graphs. The channel radius is non-dimensionalized using $\zeta=r / R$, where $\mathrm{R}$ is the radius of the channel and $\mathrm{r}$ is the radial position relative to the center of the channel. The $\mathrm{x}$-axis is the non-dimensional velocity, $\mathrm{U} / U_{\max }$, where $U_{\max }$ is the centerline velocity and $\mathrm{U}$ is the calculated velocity at position $\mathrm{r}$. Table 4.1 has the information for each test run. 

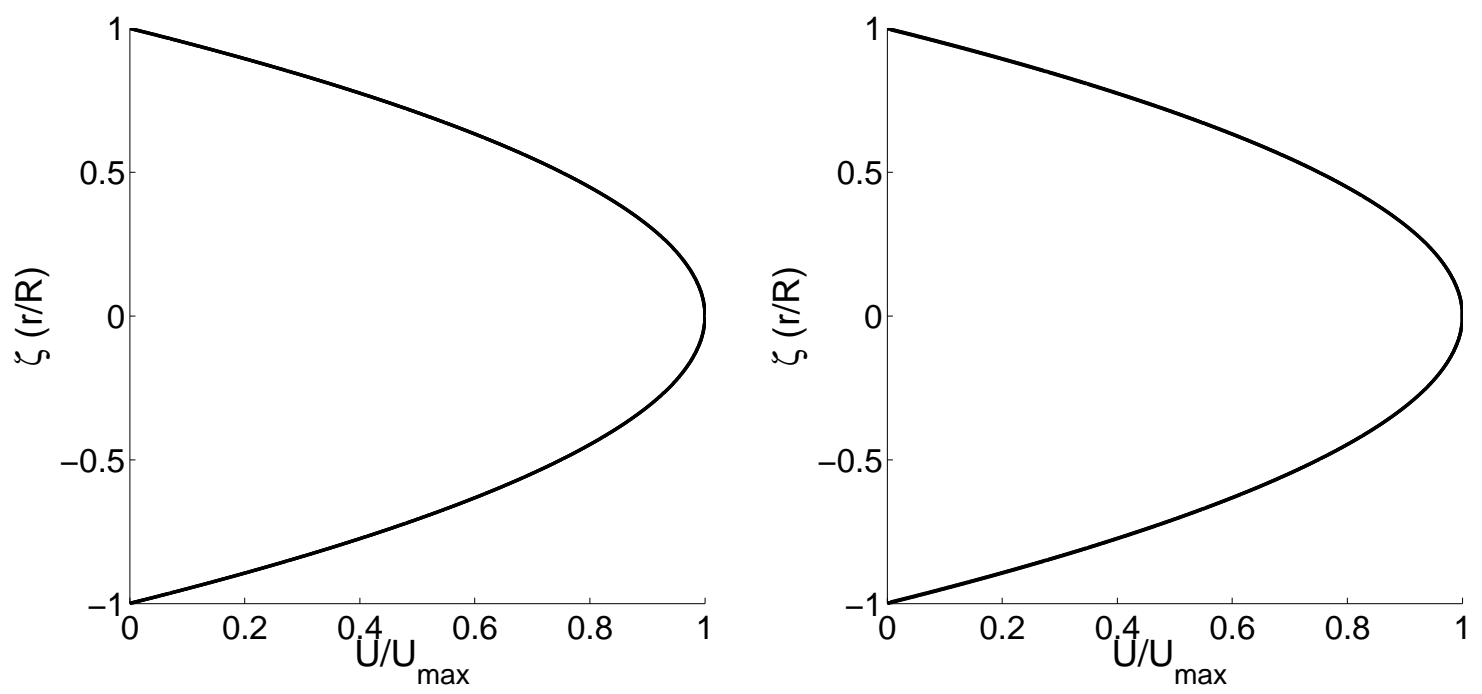

Figure 4.2. Flow profiles for the dry round channels, the profiles are for the bounding Ca numbers for the data. Left (a), is calculated using the low Ca number, 4E-5 and right (b), uses high Ca number, 2E-4.

Both flow profiles appear to overlap one another for each graph and its not until the magnification is increased near the wall that the divergence appears, Figures 4.3 and 4.5. Couette flow in the film has little effect on the overall flow profiles. The films, calculated from the Bretheron (1961) relationship, are small compared to the radius and the natural $\log$ term for $U_{A B} 4.4$ and $u_{F i l m A} 4.2$ is approximately zero. 

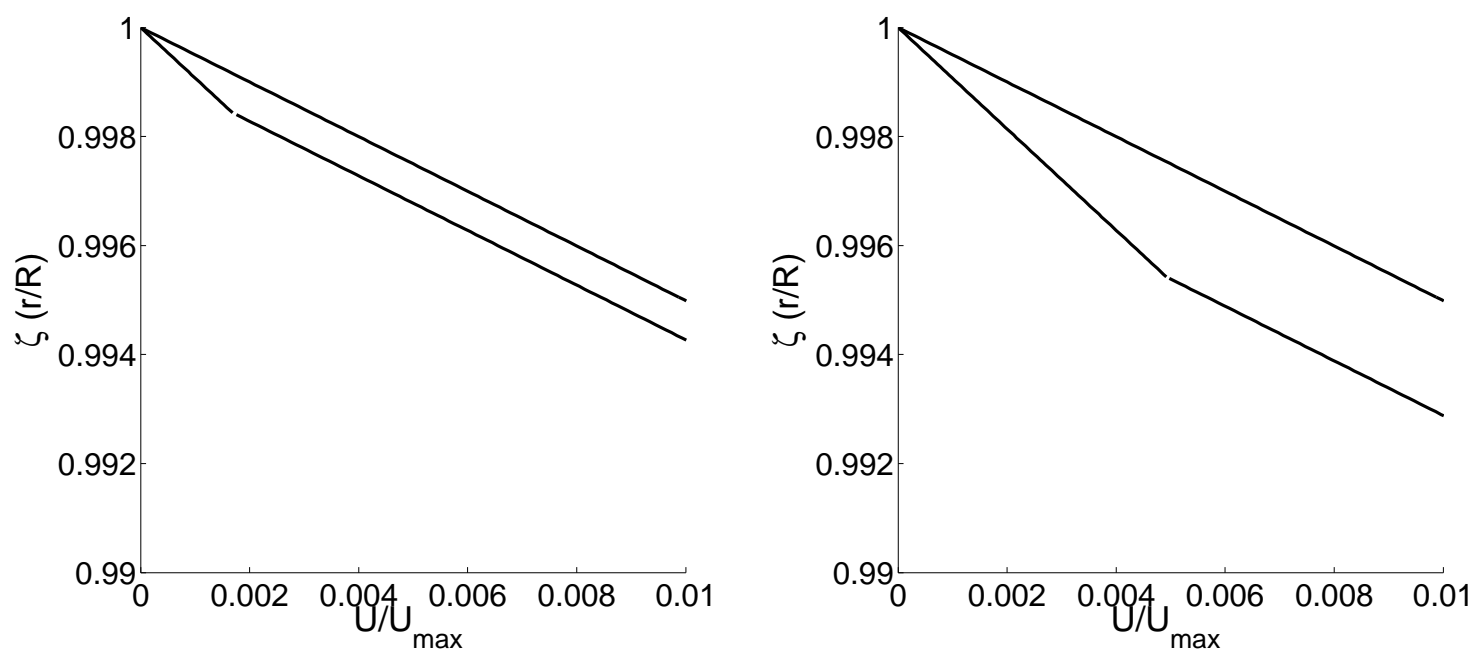

Figure 4.3. The flow profiles close to the wall for the dry round channels. (a) Left, is for the low Ca number, 4E-5 and (b) right, is the high Ca number, $2 \mathrm{E}-4$.

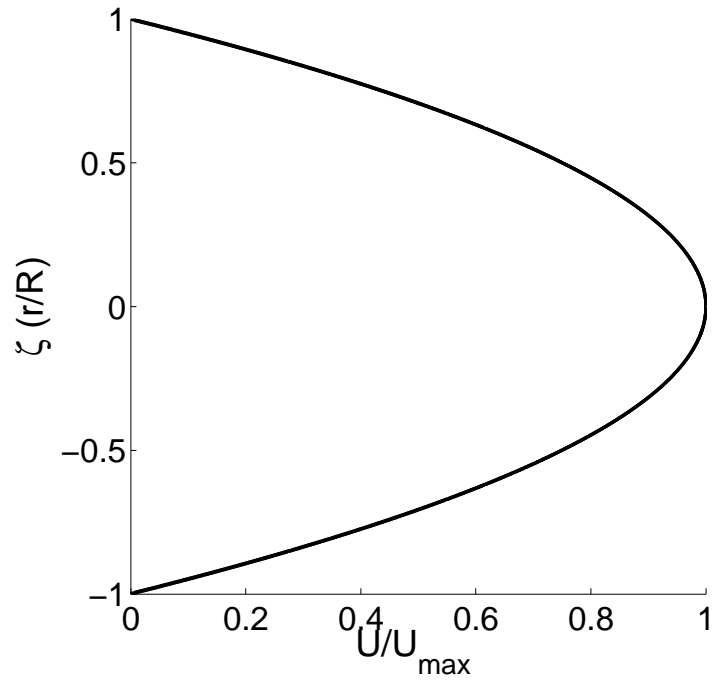

(a)

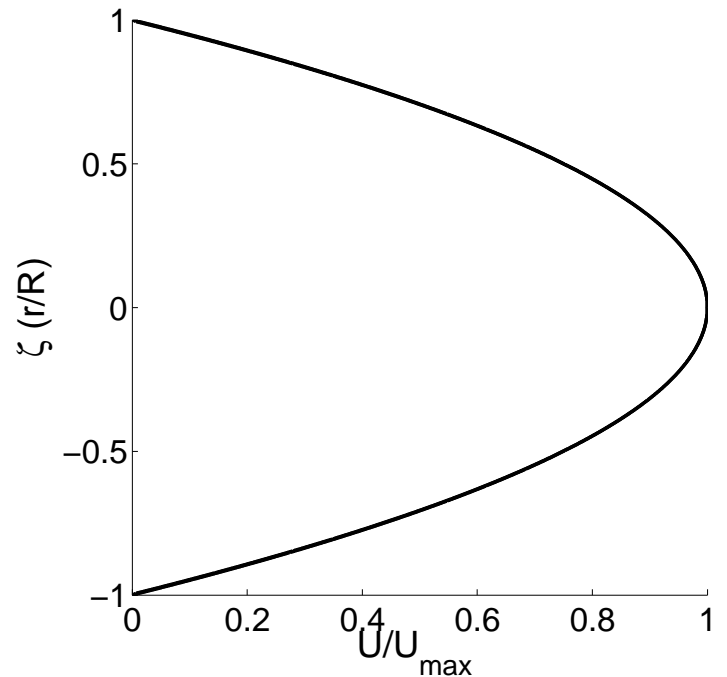

(b)

Figure 4.4. Flow profiles for the prewet round channels, the profiles are for the bounding Ca numbers for the data. Left (a), is for the low Ca number, 2E-4 and right (b), is the high Ca number, 3E-4. 


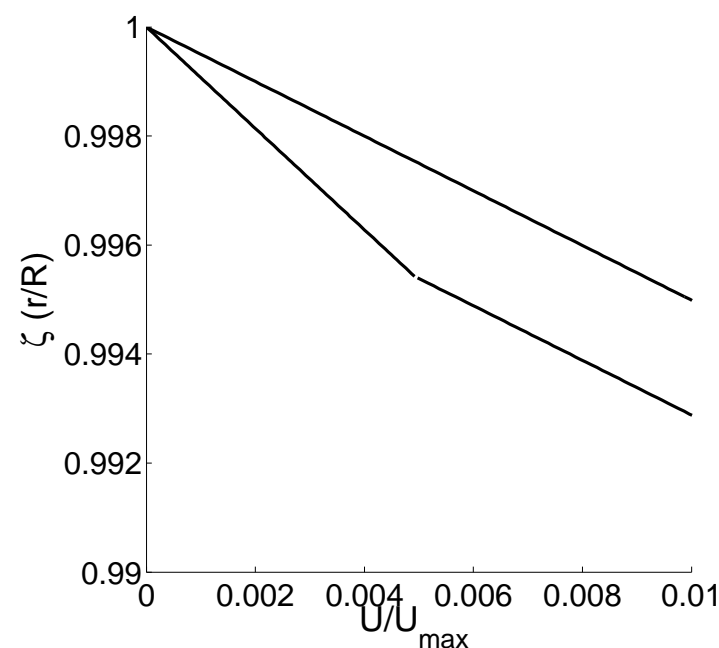

(a)

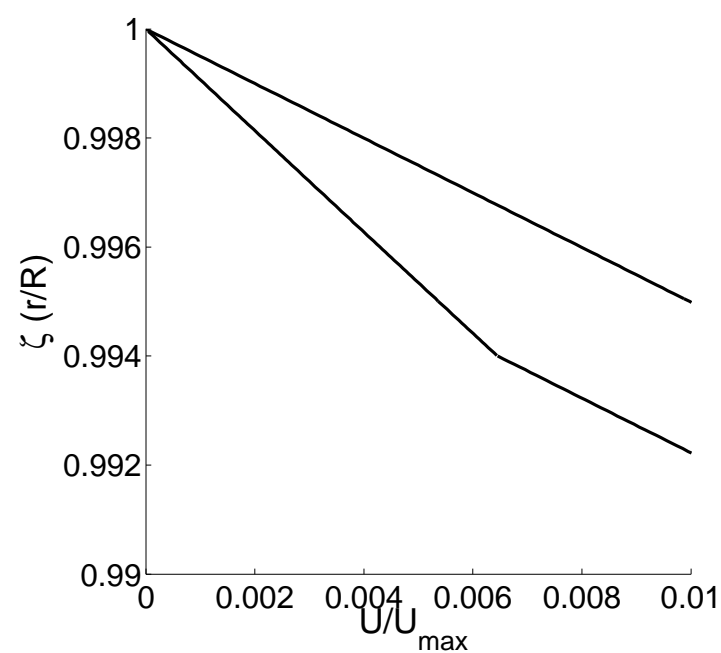

(b)

Figure 4.5. The flow profiles close to the wall for the prewet round channels. Left(a), is for the low Ca number, 2E-4 and right(b), is the high Ca number, 3E-4. 


\subsubsection{Square}

Relationships for the velocity profiles in the square channels were found in the same manner as the round channel. The same equations were used for the solution of the round channels with the exception of the radius term. A relationship for the distance from the center of the square channel to any point was used, producing a psuedo radius, $s$.

$$
s=\sqrt{x^{2}+y^{2}}
$$

Using s for the radial term the equations for the velocities in the square channels are:

$$
\begin{gathered}
u_{\text {CoreA }}=U_{\max }\left[1-\frac{s^{2}}{S_{o}^{2}}\right] \\
u_{F i l m A}=U_{A B} \frac{\ln \left(\frac{S_{o}}{s}\right)}{\ln \left(\frac{S_{o}}{S_{f}}\right)} \\
u_{C o r e B}=\left(U_{\max }-U_{A B}\right)\left(1-\frac{s^{2}}{S_{f}^{2}}\right)+U_{A B} \\
U_{A B}=\frac{U_{\max }}{\left(1+\frac{1}{2} \frac{\mu_{A}}{\mu_{B}} \frac{1}{\ln \frac{S_{o}}{S_{f}}}\right)}
\end{gathered}
$$

Full derivation of the equations are in Appendix C.

Velocity profiles for the square channels were calculated at varying points down the channel due to the dependence of the distance equation, $s$, on the two variables $x$ and $y$. The position on the wall, $x$, was held constant at various points down the channel and the value $y$ was varied from the center of the channel to the wall. Figure 4.6 illustrates the geometry of the square channel used for the calculations. The channel cross-section is divided into eight symmetric regions. The models use a single region shown in the right picture in Figure 4.6. The schematic shows the distances in nondimensional form, $\sigma=s / R$ where $R$ is hydraulic radius of the channel, which is half of the wall length for a square. The $o$ and $f$ subscripts indicate the distance from the center to the wall and the transition from film to core, respectively. The transition is broken into two parts, the flat region has a constant film thickness of $h_{o}$ and the corner has a varying film thickness of $h_{c}$ with a radius of $R_{c}$.

The non-prewet channel was not modeled because of the erratic data obtained during experimentation. Because of the corner flow, the channel is never truly dry and amount of liquid pumped into the corners depends on the time between the deposition of the first fluid and the introduction of the trailing slug, channel defects, and channel orientation during experimental setup.

Plots of the slug profiles are split between the leading and trailing slugs due to the amount of information presented. Bislug velocity profiles are plotted in Figure 4.8 for the hich $\mathrm{Ca}$ number and Figure 4.7 for the low Ca number. Each of the figure 

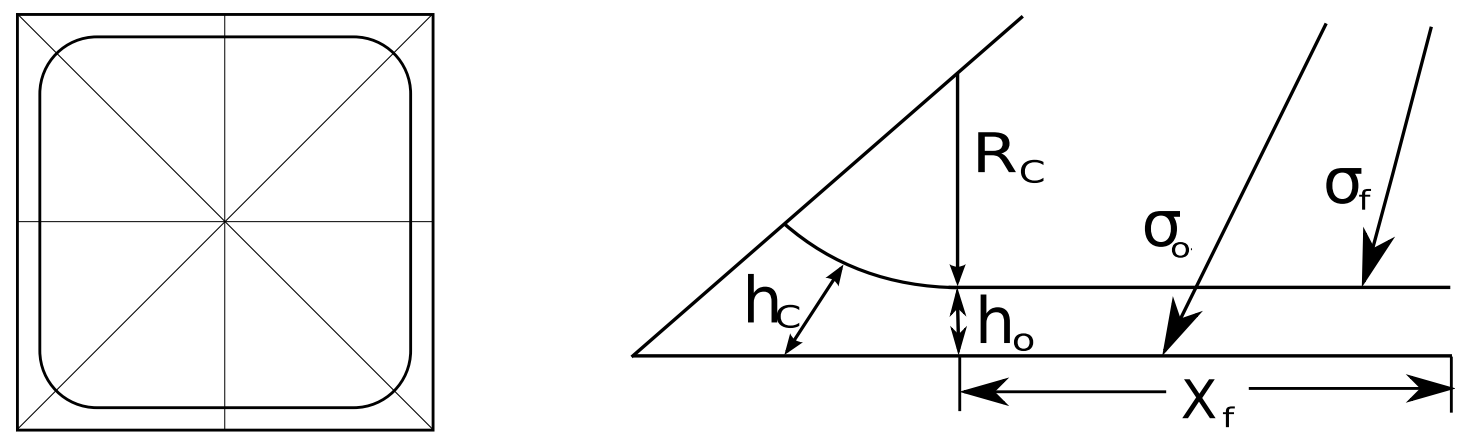

Figure 4.6. Geometry for the square channels. The square profile has eight symmetric sections, each of the sections has the profile on the right

Table 4.2. Parameters for Square graphs

\begin{tabular}{|c|c|c|c|}
\hline Test Runs & Ca & $U_{\max }(\mathrm{mm} / \mathrm{s})$ & $h_{o}(\mu \mathrm{m})$ \\
\hline Prewet Square Low Ca (a) & $2 \mathrm{E}-3$ & 5.3596 & 10.615 \\
Prewet Square High Ca (b) & $4 \mathrm{E}-3$ & 10.7191 & 16.851 \\
\hline
\end{tabular}

sets consists of (i)the flow in Core A (without film), (ii)Core B and Film A,(iii)the location of the profiles in the channel and (iv)the velocity of the liquid-liquid interface. Both the profiles in Core A and Core B/Film A have the same general shape but the affects of the film can be seen clearly which differs from the round channels where the Couette flow had little affect. Since the film was a constant height in the flat region the solutions for the profiles are merely stretched or enlongated from profile to profile in the direction from the center to the wall until the transition into the corner. The film velocities are no longer constant in the corner due to the changing film thickness which is now dependent on the profile's position in the channel. If the Concuss-Finn criteria had not been met then the film thickness would remain constant along the channel. The films were calculated using the Bretherton relationship, the increased importance of the Couette flow may be attributed to the order of magnitude increase in Ca number from round prewet to square prewet. The increased length of the Couette flow can be seen in Figures 4.9. For the round channels the Couette flow ended before $U / U_{\max }=.01$, in a square channel the Couette profile has a $U / U_{\max }$ ratio of at least .02. This is due to the magnitude increase in velocity in the system which also creates a thicker film. Parameters for the test runs are in Table 4.2.

The radius of the film in the corner was estimated to be $10 \%$ of the channel width based on Figure 5.7 in Chapter 5. Kolb (1991) developed a relationship for calculating the radius but the properties for this setup resulted in a negative number. Kolb (1991) used higher flowrates which results in Ca numbers that are larger than those in these experiments. Also, the extrapolation of the presented graph also results in a negative 

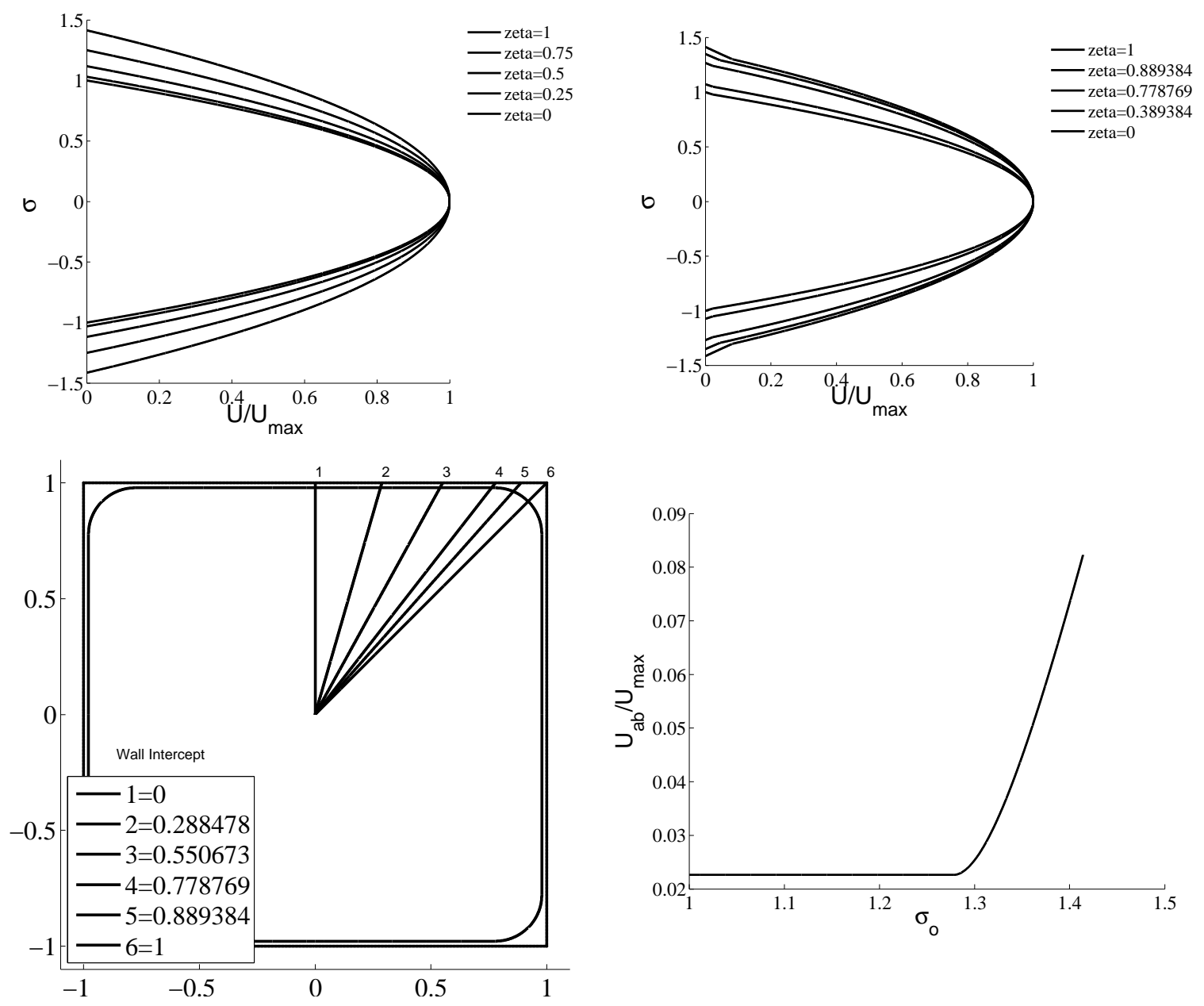

Figure 4.7. Plots for the low Ca number, 2E-4. (a) Upper left, flow profiles for Core A, no film, (b) upper right, flow profiles with the thin film, Core B and Film A, (c) bottom left, the lines where the profiles were calculate, (d) bottom right, velocity at the transition between the film and the core.

number for the low Ca numbers. 

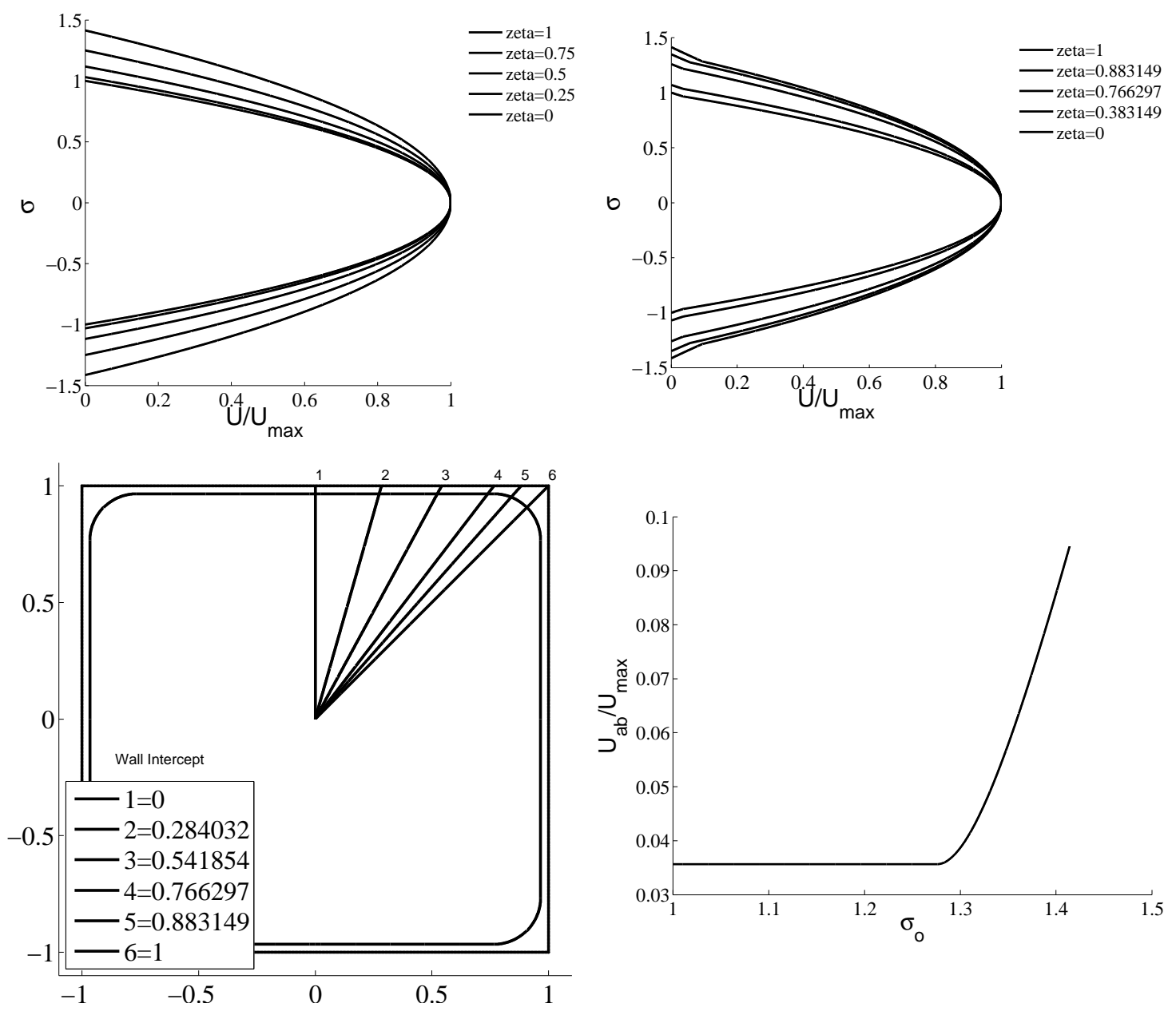

Figure 4.8. Plots for the high Ca number, 4E-3. (a)Upper left, flow profiles for Core A, no film, (b) upper right, flow profiles with the thin film, Core B and Film A, (c) bottom left, the lines where the profiles were calculate, (d) bottom right, velocity at the transition between the film and the core. 

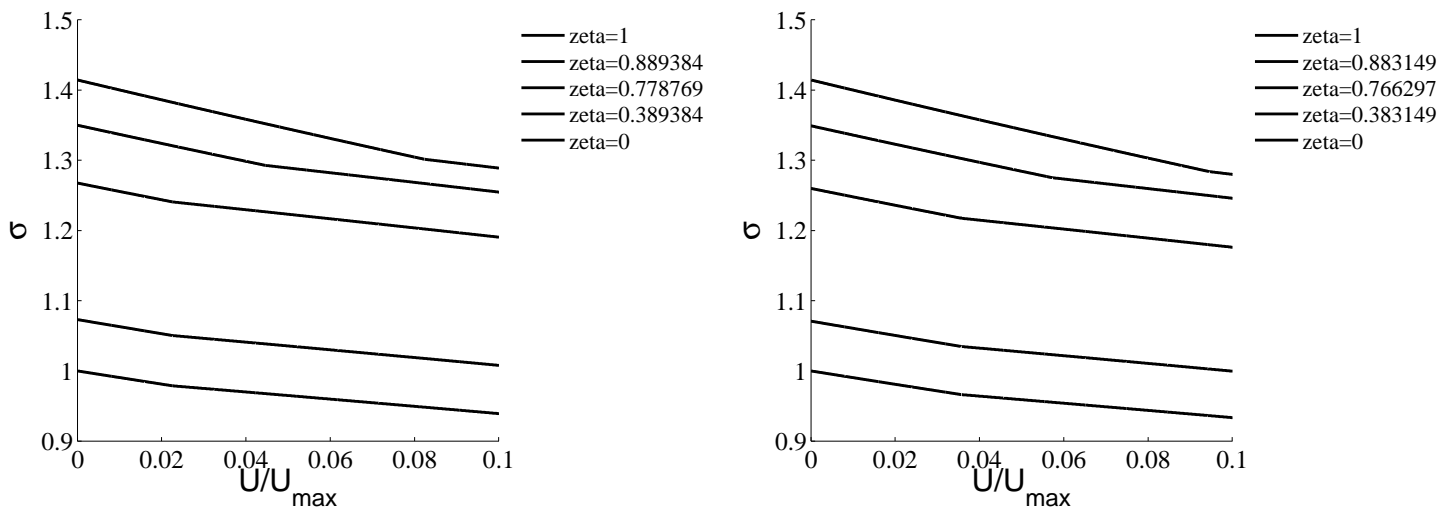

Figure 4.9. The flow profile close to the wall for the square channels. (a) Left, is the low Ca flowrate, 2E-3 and (b) right, is the high Ca number flowrate, 4E-3. 


\subsection{Force Balance}

A force balance of the pressure potential and the two dissipation terms, drag force and interface stretching, was used to develop a predicitive model for the velocity of a bislug in a microchannel. The pressure potential is calculated using the LaplaceYoung equation 2.3 and the force can be calculated by multiplying this over the area of the menisci. The drag force is the result of the shear stress and is applied to the surface area over which it acts. The interface stretching term is the result of the destruction and creation of the thin film interfaces as the bislug moves down the channel.

\subsubsection{Round}

The pressure potential of a bislug system is dependent on the differences in surface tensions of the three interfaces and the radius of the channel(equation 4.10).

$$
\Delta P=\frac{2\left(\sigma_{A}-\sigma_{A B}-\sigma_{B}\right)}{R}
$$

The net drag force is comprised of three separate components: (i) the drag in the core of slug A, (ii) the drag in the core of slug B, and (iii) drag in the thin film of fluid A that is under slug B. A thin layer of ethylene glycol, fluid A, is deposited on the walls of the microchannels before fluid B is introduced for prewet test runs. Since fluid A and fluid B are immiscible the thin film of fluid A remains intact under the slug of fluid B. Flow in both the cores of slug A and slug B are model as Hagen-Poiseuille flow due to the constant pressure potential between the menisci. Couette flow was assumed for the thin film; there is a no slip condition at the wall and a constant velocity at the interface between film $\mathrm{A}$ and slug $\mathrm{B}$. The total drag force is the sum of all three of these components.

$$
F_{\text {drag }}=F_{\text {core } A}+F_{\text {film } A}+F_{\text {core } B}
$$

The resulting equation for the drag force is equation 4.12 ,

$$
F_{\text {drag }} \approx 4 \pi \sigma_{A} L_{A} C a_{A}\left[1+2 \frac{1}{M}\left(\frac{L_{B}}{L_{A}}\right)\right], \mathrm{Ca} \leq 10^{-3}
$$

where the velocity has been non-dimensionalized using the Ca number for the leading slug, $C a_{A}=U_{\max } \mu_{A} / \sigma_{A}$ and $M$ is the viscosity ratio, $\mu_{A} / \mu_{B}$. The full derivation can be found in Appendix B.1.3.

As a bislug of fluid travels down a prewet microchannel the front meniscus destroys the air-film A interface, absorbing the energy from the surface. At the same time meniscus $\mathrm{AB}$ and meniscus $\mathrm{B}$ create new interfaces on the wall, which requires

energy and reduces the overall potential for flow in the system. A single slug flowing in a microchannel has only two menisci. The amount of energy restored from the 
destruction at the front meniscus is removed with the creation of the interface at the rear meniscus for a net interface creation or destruction of approximately zero. In a bislug system there are three different surface tensions at the different menisci resulting in a non zero energy exchange. For this setup there are two interfaces created and one destroyed which, depending on the surface tensions, produces a net energy change in the system. Equation 4.13 is the final form of the stretching force and it is based on the summation of the three stretching terms across the the bislug.

$$
F_{\text {stretch }}=\sigma P_{\text {e,coat }}=2 \pi R\left[\left(1-\frac{h_{A}}{R}\right)\left(\sigma_{A B}+\sigma_{B}-\sigma_{A}\right)-\sigma_{B} \frac{h_{B}}{R}\right]
$$

where $P_{\mathrm{e}, \text { coat }}$ is the perimeter of the film cross-section.

Equation 4.14 is the final form of the model and has been non-dimensionalized using the Capillary number. The full derivation can be found in Appendix B. From the model it can be seen that the Capillary number is a function of the surface tensions of the two fluids, the radius of curvature, and the lengths of the slugs. The final form of the model is equation 4.14 .

$$
C a_{\max } \approx\left[1-\left(\frac{\sigma_{A B}+\sigma_{B}}{\sigma_{A}}\right)\right]\left(\frac{R}{L_{A}}\right)\left(\frac{1}{1+2 \frac{\mu_{B}}{\mu_{A}} \frac{L_{B}}{L_{A}}}\right), \text { for } C a<10^{-3}
$$

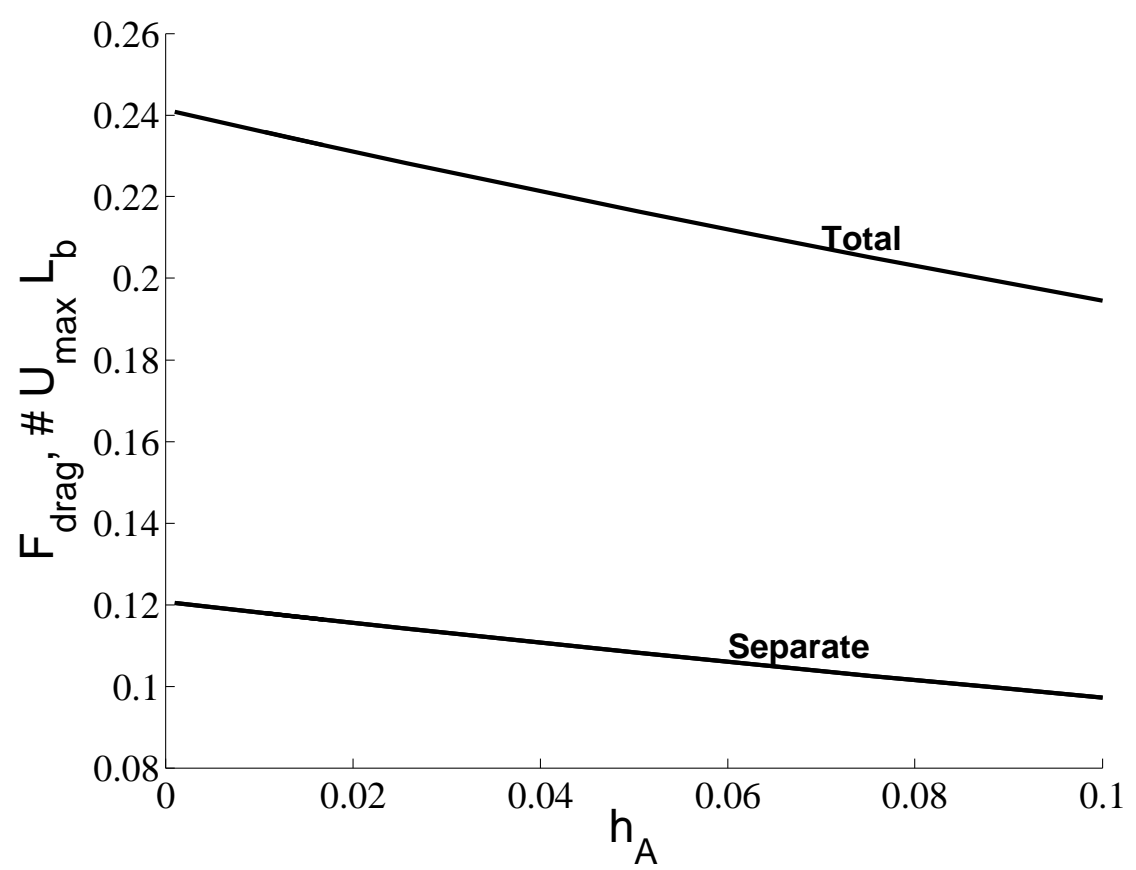

Figure 4.10. Drag forces in a round channel, because of the small change in surface area the drag forces overlap.

The drag force in round channels has been solved for the Core B and Film A as a function of film thickness. Values for the drag force are the coefficient multiplied 
by the centerline velocity and the slug length. Both the drag from the film and core plotted one the figure, left plot in Figure 4.10. Due to the small change in surface area between the film and the core, the drag force in the system are close in value and the lines overlap. The film thickness is small and does not change the overall area, $2 \pi R$ compared to $2 \pi\left(R-h_{o}\right)$ for the core and film respectively.

\subsubsection{Square}

The summation of forces in a square microchannel used the same approach that was used for the round microchannels, which is a balance between the motive, drag and stretching forces. Though the channel is square the menisci of the slugs have a sperical shape, which produces the same pressure potential resulting in the same equation as the round channels, equation 4.10 , where $\mathrm{R}$ is the hydraulic radius which for a square is half of the side wall length.

As with the round geometry the square channels also have interface destruction and creation as the bislug travels down the channel. Each of the menisci have a two part profile, a flat and curve section, Figure 4.6.

$$
F_{\text {stretch }}=8\left(F_{\text {stretch flat }}+F_{\text {stretchcorner }}\right)=8 \sigma\left(P e_{\text {flat }}+P e_{\text {corner }}\right)
$$

The multiplier of eight is used because the channel was broken into eight symmetric parts, Figure 4.6. The final form of the stretching term is

$$
2 \pi R\left[\left(1-\frac{h_{A}}{R}\right)\left(\sigma_{A B}+\sigma_{B}-\sigma_{A}\right)-\frac{x_{f}}{R}\left(\sigma_{A B}+\sigma_{B}\right)-\sigma_{B} \frac{h_{B}}{R}\right]+8 x_{f}\left(\sigma_{A B}+\sigma_{B}\right)
$$

Where $x_{f}$ is the distance from the center of the wall to the transition between the flat and corner regions. The full derivation is in Appendix C.1.1.

The summation of the forces for the square channel does not result in a closed form solution like the round channels. The drag term requires numerical integration for solution due to the complex form of the shear stresses. The term that is numerically integrated is dependent on the viscosity ratio. Results for the drag are unique for each fluid pair. Equations for the drag in the three regions of bislug are below. The results for the drag in Film A and Core B are combined and the numerical value from the integration is contained in the variable $\alpha$. A closed form solution is available for Core A but the variable $\beta$ was used to keep the equations consistent.

$$
\begin{aligned}
F_{\text {drag }_{\text {Core } A}} & =\beta \mu_{A} U_{\max } L_{A} \\
F_{\text {drag }_{\text {Core B }}}+F_{\text {drag }} \text { FilmA } & =\alpha U_{\text {max }} L_{B}
\end{aligned}
$$

Table 4.3 has the coefficients from the integration at the two boundary Ca numbers. A upper and lower bound for the data represented on Figure 4.11 as the circle and square, low and high Ca number respectively. The drag force in the channels decreases 
as the film thickness increases. This is expected because the simple form of the shear stress in the film is dependent on the thickness of the film and the shear decreases with increasing film thickness. There is a clear separation between the shear stress in the film and the fluid core. This is due to the change in geometry from the flat film to the corner, which decreases the area over which the shear stress in fluid B acts.

Table 4.3. Coefficients for $F_{d r a g}$ in the square channels

\begin{tabular}{|l|l|l|l|}
\hline Ca Number & $h_{o}(\mu \mathrm{m})$ & $\alpha(\mathrm{kg} / \mathrm{m} \mathrm{s})$ & $\beta$ \\
\hline 2 E-3 & 10.6 & 0.52567 & 14.1020 \\
$4 \mathrm{E}-3$ & 16.9 & 0.51926 & 14.1020 \\
\hline
\end{tabular}

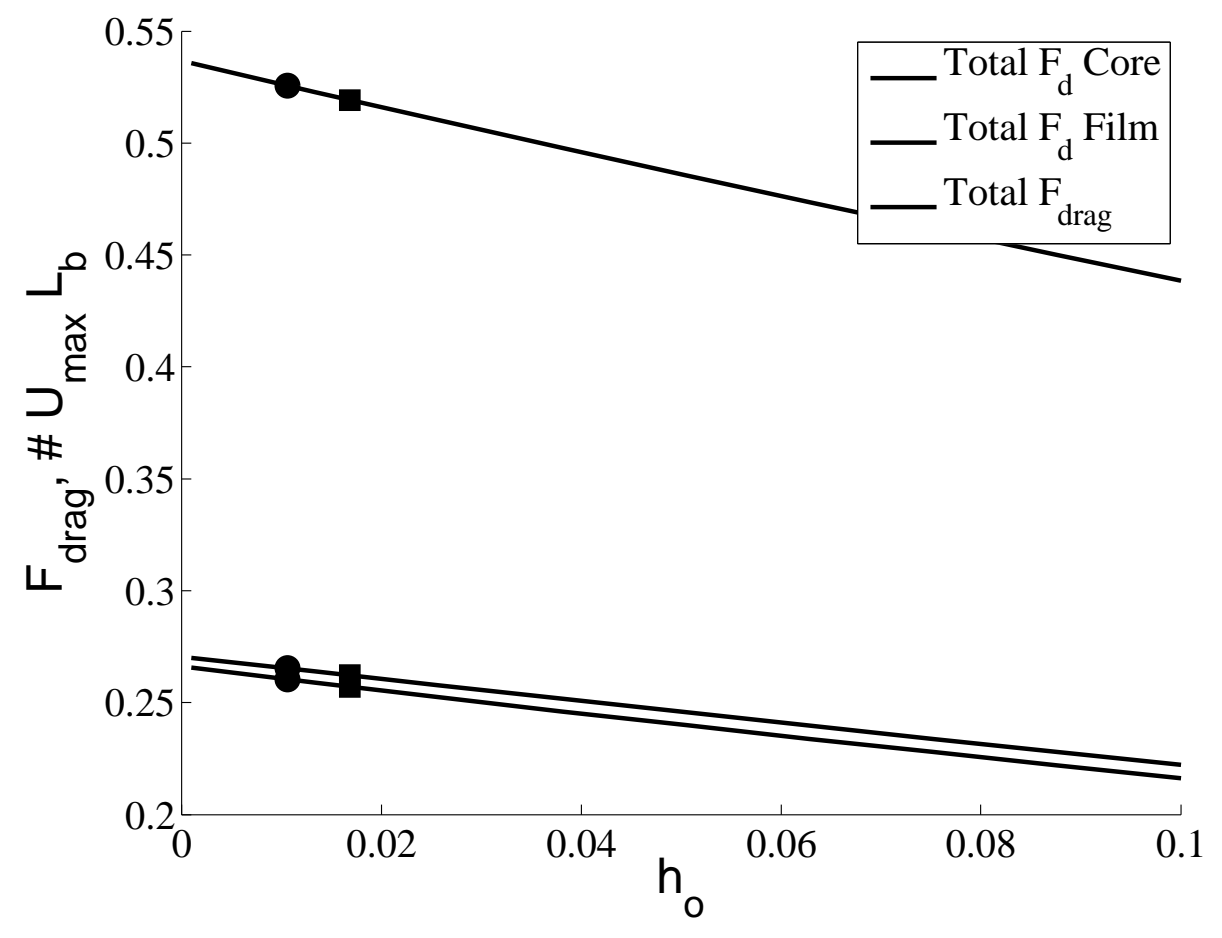

Figure 4.11. The drag in a square channel, the circle and square are the boundaries used for data comparison, low Ca and high Ca.

The resulting model from the force balance is equation 4.18. The velocity has been non-dimensionalized using the Ca number with respect to fluid A. The film thickness for fluid $\mathrm{A}, h_{A}$, is approximated from the Bretherton relationship 2.5. The film for 
fluid B was assumed to be close to zero, this has a minimal effect on the model.

$$
\begin{aligned}
& C a_{\max A}=\frac{U_{\max } \mu_{A}}{\sigma_{A}}= \\
& \frac{2 \pi R}{L_{A}} \frac{\left[\left(\frac{\sigma_{A B}+\sigma_{B}}{\sigma_{A}}\right)\left(2-\frac{h_{A}}{R}\right)+\frac{x_{f}}{R}\left(1-\frac{4}{\pi}\right)\left(\frac{\sigma_{A B}+\sigma_{B}}{\sigma_{A}}\right)\right]}{\left(\frac{\alpha}{\mu_{A}} \frac{L_{B}}{L_{A}}+\beta\right)}
\end{aligned}
$$

Similar to the round channel model the Ca is dependent on the radius of the channel, surface tensions and the slug lengths. This model is also dependent on the film thickness, which if unknown has to be iterated because of the film thickness dependence on the Ca number and the transition length between the flat and round area. If the slug is round and the transition length is zero then the model takes on a similar form as the round channel. The difference is the factor $2-h_{A} / R$ which is removed in the round channel because of the thin film assumption. A model was also developed without the inclusion of the stretching term. The results will be used to compare the role of the stretching term.

$$
C a_{\max A}=\frac{2 \pi R}{L_{A}} \frac{1-\left(\frac{\sigma_{A B}+\sigma_{B}}{\sigma_{A}}\right)}{\frac{\alpha}{\mu_{A}} \frac{L_{B}}{L_{A}}+\beta}
$$




\section{Chapter 5}

\section{Results and Discussion}

\subsection{Round Microchannels}

Velocities for both the prewet and dry microchannel test runs were non-dimensionalized using the Capillary number with reference to the ethylene glycol (equation 5.1). A non-dimensionalized length scale $L_{A} / \mathrm{R}$ is where $\mathrm{R}$ is the radius of the channel. For the data discussed $\mathrm{R}=0.469 \mathrm{~mm}$.

$$
C a=\frac{\mu_{A} V}{\sigma_{A}}
$$

Data from both the prewet and dry test runs are plotted on Figure 5.1. There is a noticable difference in velocity between the prewet and dry microchannels. Bislug flow in a prewet channel is approximately 2-3 times faster than the dry channel. The average capillary number for dry and prewet tests are $1 \cdot 10^{-4}$ and $2 \cdot 10^{-4}$, respectively. Differences in velocity can be attributed to energy dissipation associated with the dragging of the contact line across a dry surface. A prewet channel has a thin layer of fluid deposited on the walls before the bislug is created as described earlier. This coating reduces the energy dissipation with the moving contact line. In both systems the pressure difference between the two menisci is the same, $\Delta P=40.1 \mathrm{~Pa}$. As such, this type of experiment may provide a way to study energy dissipation near a moving contact line if a proper model can be developed.

Bislug flow was previously modeled as classic Hagen-Poiseuille flow [4, 5]. This model, to compare to the model produced in this work, has been non-dimensionalized into equation 5.2.

$$
C a=\frac{\mu_{A} V}{\sigma_{A}}=\frac{R}{4 L_{A}}\left(\frac{\sigma_{A}^{*}}{1+M^{*} L^{*}}\right)
$$

Where $\sigma_{A}^{*}=1-\sigma_{A B} / \sigma_{A}-\sigma_{B} / \sigma_{A}, M^{*}=\mu_{B} / \mu_{A}$ and $L^{*}=L_{B} / L_{A}$. This equation over predicts the values for the Ca number calculated using the data collected from this investigation. Another equation was developed from the same authors [5] that includes the dissipation from the moving contact line. This new equation uses a $\mathrm{V}^{*}$ term to represent the maximum velocity of the bislug. The value for $\mathrm{V}^{*}$ could not be 


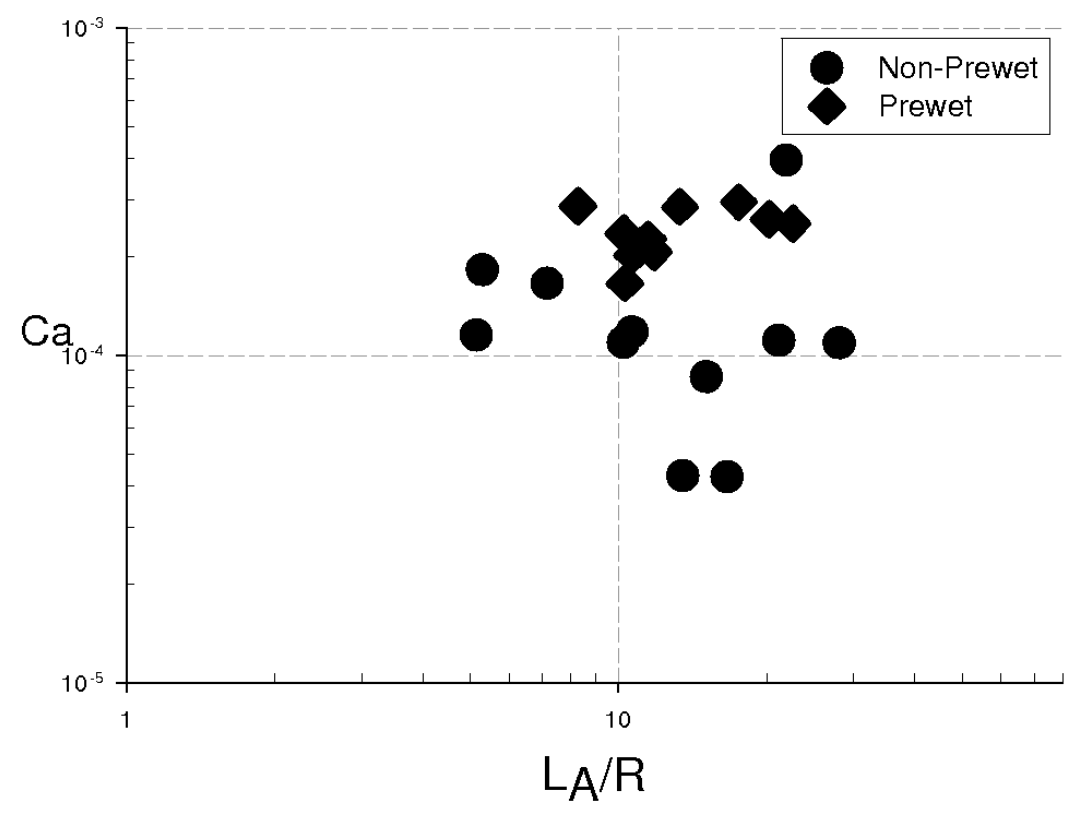

Figure 5.1. The prewet and non-prewet round channel data.

reproduced using the data from the paper. $\mathrm{V}^{*}$ appears to be an empirical fit for the data and would vary with channel geometry and fluid pairs.

Bislug flow in round channels can be used to study the energy dissipation mechanism of slug flows. There are four primary dissipation mechanisms in slug flow; hydrodynamic interaction of proximate menisci, moving contact line, wall shear stress and interface stretching. The wall shear stress was modeled based on Hagen-Poiseuille flow. As the slug grows in length, the wall drag will begin to dominate the dissipation mechanisms. The shear stress in the long slugs will dominate other dissipation mechanisms and this may provide a means of isolating this particular energy dissipation mechanism. A short slug is dominated by menisci interaction. As the slug shortens the recirculation present at each menisci will begin to interact with each other and dissipate energy. The force from the interface stretching is dependent on the film thickness and increases in importance as the the slugs decrease in length. The final dissipation mechanism is the dragging of the contact line across the channel and it is present at each menisci as the bislug flows down a microchannel.

The moving contact line dissipation is present in all slug flow, energy is lost as the menisci moves across the channel. As the slug moves down the channel, fluid is deposited from the menisci to the walls. This produces recirculation around the area where the meniscus contacts the wall reducing the energy in the system. As the front meniscus flows down the prewet microchannel the air-Fluid A thin film interface is 
destroyed. Energy is transfered from the stretched interface into the system as the front meniscus and thin film form a continuous interface. The amount of energy recovered is a function of the surface tension and the stretched area. The middle and rear menisci create new interfaces as the bislug travels down the channel. The middle meniscus produces the same thin film interface that is destroyed by the front meniscus. The rear menisci of Fluid B creates a thin over the existing thin film of Fluid A. This stretching term is normally neglected in single slug flows but it has a significant effect on the bislug system.

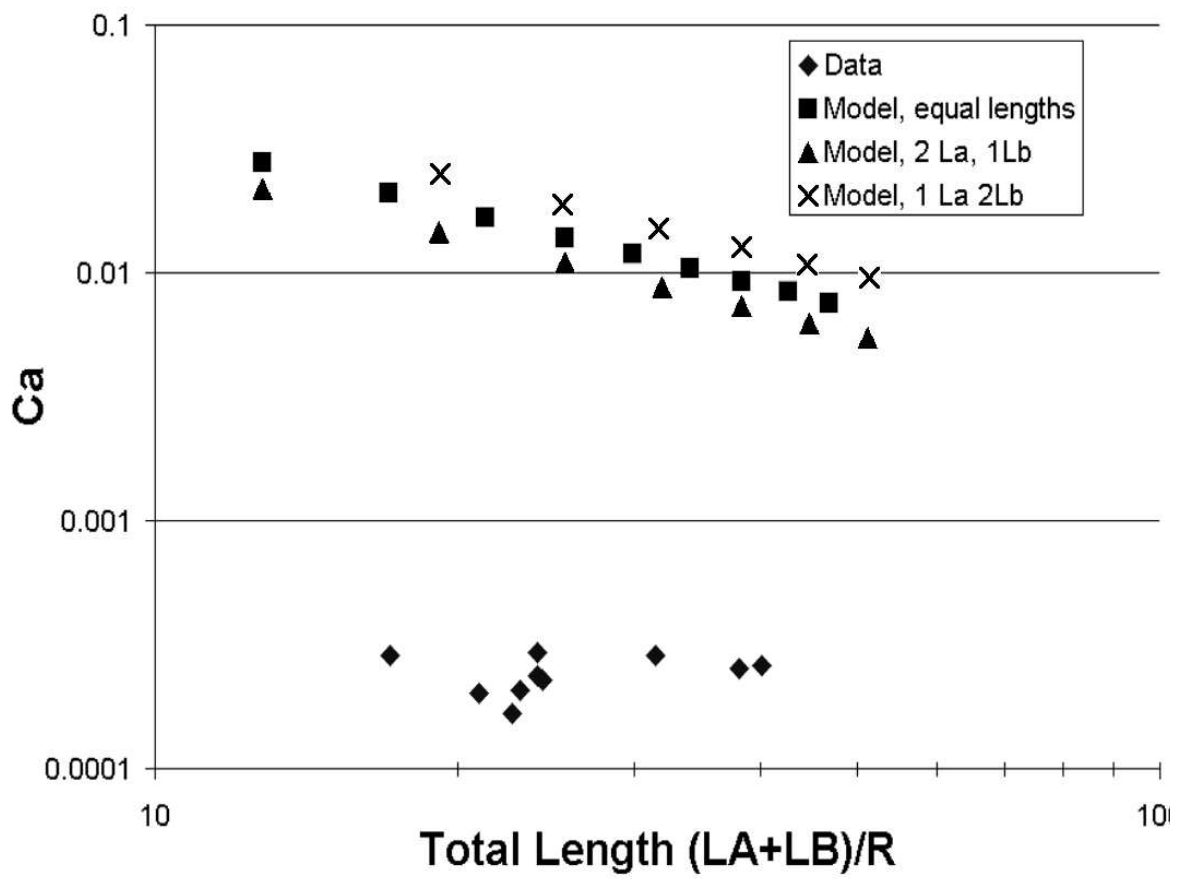

Figure 5.2. Ca numbers for both the model and the Prewet data, the model is based on equation 4.14 using a ratio of $L_{B} / L_{A}=.5,1,2$

There is a magnitude or more difference in the values for the Ca numbers between the model and the data collected for the round channels. Experimental data is plotted against the model in Figure 5.2, the plot uses values of $1 / 2,1$, and 2 for the ratio of the slug lengths. The differences between the model values and the actual $\mathrm{Ca}$ numbers can be attributed to the additional dissipation terms that are not present in the model. Menisci interactions begin to dominate the system as the slugs decrease in length and drag force decreases. Energy dissipation at the contact line is present in all slug flow and will produce an offset to the experimental data. As the slugs for both Fluid A and Fluid B increase in length the experimental data will approach the values predicted by the model per the increase in viscous stresses. The model cannot predict the $\mathrm{Ca}$ number of short bislugs due to the magnitude of the hydrodynamic 
interactions. A better understanding of this energy dissipation mechanism is needed. Visualization data is needed near the menisci to accurately model this phenomenom.

\subsection{Square Microchannels}

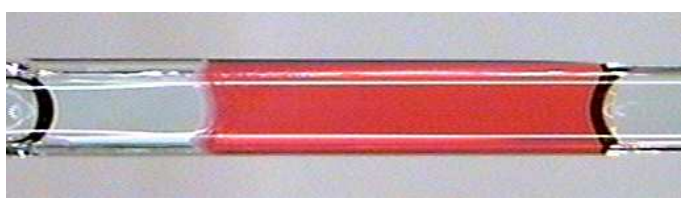

(a)

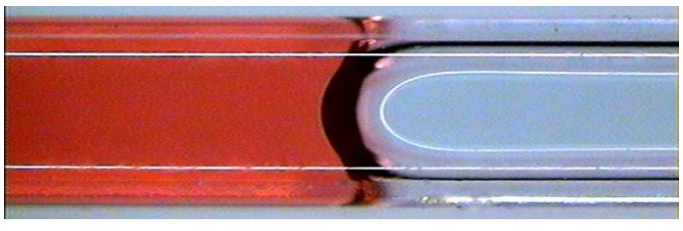

(b) .

Figure 5.3. a) Bislug in a square channel. b) Fluid retention in the corner of a prewet square microchannel

Initially both dry and prewet cases were examined for bislug flow in square microchannels. Data from the dry microchannel test were found to be erratic. Since the microchannels satisfy the Concus-Finn criteria for corner flow, dry channels could not be maintained when the ethylene glycol was introduced to the square microchannels and flow occured spontaneously in the corners. The fluid retention in the corners can be seen on the right side of Figure 5.3(b). This, in effect, prewetted the corners and partially wetted the walls of microchannels. The partially prewet microchannels produced data having a large scatter which can be seen in Figure 5.4. It was later decided to focus the attention on prewet flow in square microchannel, which is the scenario that would be used in a micropump.

Velocities for bislug flow in square microchannels are presented in terms of the Ca number. The velocities were non-dimensionalized using the same equation used for round tests, equation 5.1. Figures (5.5-5.6) show the Capillary number for the ethylene glycol as a function of the ethylene glycol and silicone oil slug lengths. The term $L / R$ is a ratio of the slug length, either $\mathrm{A}$ or $\mathrm{B}$, to the radius of the channel, $R=0.5 \mathrm{~mm}$.

As the bislug moves, fluid is deposited on the walls of the channel and in the corners. Relative velocities for the front mensicus are slower than the velocities for the rear due to the deposition of fluid into the corers. As the slug coats the channel, material from the front of the meniscus is used which reduces the overall apparent velocity for the front mensicus. The coating thickness of the ethylene glycol has a maximum thickness in the corners and decreases in thickness until it reaches the transition length, $x_{f}$, Figure 4.6, after the transition the thickness remains constant. Using a darkfield imaging technique the amount of sidewall that is affected by the corner can be seen. The light was adjusted until the distance between the two dark bands remained constant, Figures 5.7(a-c). The volume of the fluid that is deposited can be determined by knowing the before and after lengths of the slug and the distance 


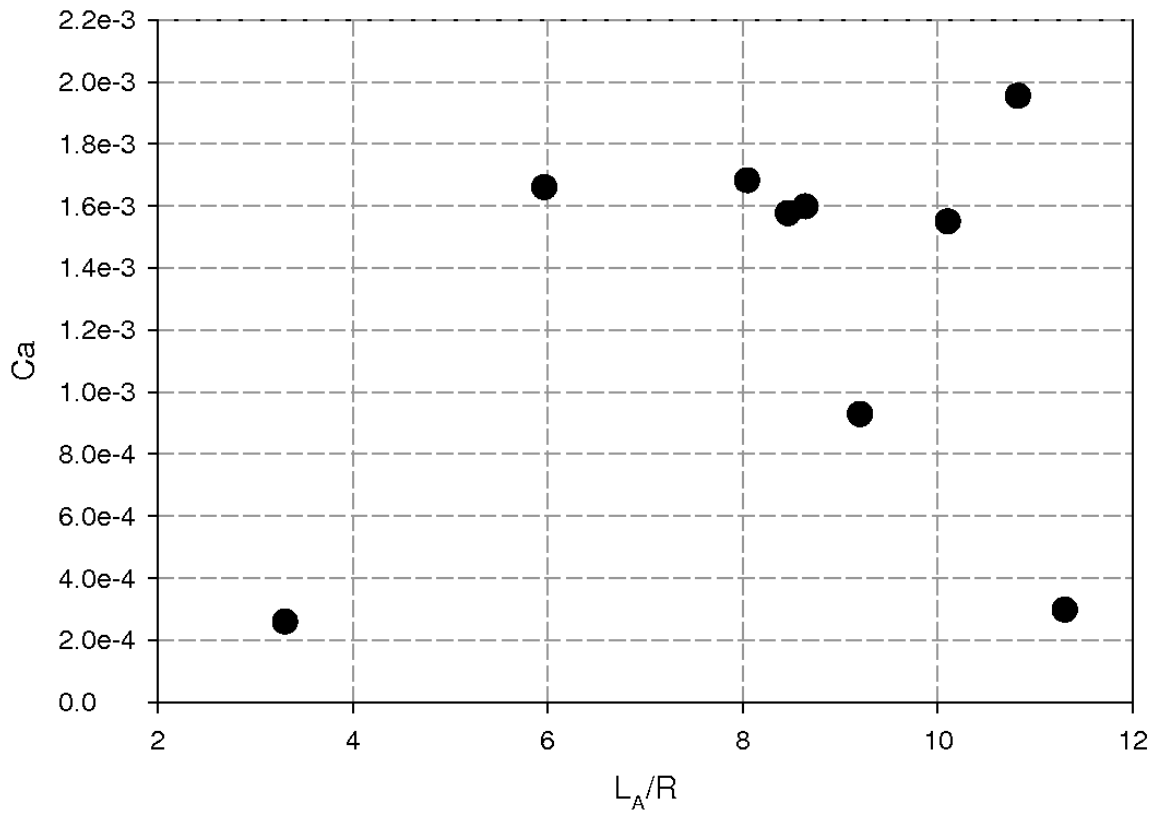

Figure 5.4. Graph of Ca for square dry channel

traveled. The film thickness can be estimated from the volume deposited and the length measured from darkfield images.

Bislug flow in a square microchannel has a faster response time when compared with channels of circular cross-section, Figures 5.1, 5.5, and 5.6. The Capillary number in a prewet square microchannel is at least an order of magnitude greater than in round microchannels. Both systems have approximately the same pressure potential, the round was $\Delta P=40.1 \mathrm{~Pa}$ and the square $\Delta P=37.6 \mathrm{~Pa}$. The square channels have a hydraulic diameter of $1 \mathrm{~mm}$, which results in a lower pressure because of the larger radius of curvature, and should exhibit a slower response. However the affects of the corners result in a fster flow(equation 4.10).

Though the overall drag coefficient in a square channel is twice as much in the round channels, see Figures 4.10 and 4.11 for comparison, the response time is faster. A square channel, due to geometry, has a larger surface area for the viscous forces to act over resulting in a larger drag coefficient. Fluid retention in the corners may act like "rails" for the slugs to travel on reducing the energy dissipation associated with a moving contact line due to the increase in fluid thickness in and near the corners. The reduction of the contact line may be the reason for the faster response. The developed model for the round channel overpredicts the response time of the bislug due to the exclusion of the contact line dissipation which appears to dominate the round channel. The losses associated with menisci interaction are still unknown and 
a reduction of energy dissipation in the this area cannot be determined.

The velocity of the bislug system has a dependency on the length of ethylene glycol while the length of silicone oil produces secondary effects. Figures 5.5 and 5.6 are the Ca numbers of the front and rear menisci as a function of slug lengths. From these figures, a correlation between slug length and speed can be seen for ethylene glycol. However, there is not a dependency between the silicone oil slug length and Ca number. This is due to the different fluid properties, the kinematic viscosity of the ethylene glycol is 16 times greater than that of the silicone oil. The viscous dissipation in the silicone oil slug is negligible compared to the ethylene glycol. The plots show the ratio between $\mu_{B}$ and $\sigma_{B}$ is small enough that the velocity term has little effect on the Capillary number for the silicone oil. The plots also show the same data that is presented in Figures 5.5 and 5.6.

A model was developed to predict the response of a bislug in a square microchannel, Chapter 4. Moving contact line, menisci interaction, shear stress at the wall, and interface stretching are the primary forms of energy dissipation found in bislug flow, with the latter being more applicable to systems with varying menisci surface tension. Dissipation at the contact line is caused by the menisci being dragged across the channel wall. It has a constant affect on the system and is independent of slug length. As a slug gets longer shear stress becomes the dominant energy dissipation mechanism due to the increase in wall area. Short slugs, less than 3 diameters, have interactions between menisci. As the slug decreases in length the affect of of the shear stress decreases. Figures 5.8 and 5.9 show the regions of dissipation for the viscous drag, menisci interaction and moving contact line. Zone 1 is affected by contact line and menisci interations, Zone 2 is affected by all three dissipation mechanisms, and Zone 3 is affected by contact line and shear stress. It may be possible to study each of these mechanisms using bislug flow and a flow visualization technique, a force balance similar to the one developed for flow in a round channel will be developed.

Figure 5.11 compares the Capillary numbers from the expirimental data to the Capillary numbers predicted from the developed model. Predictions from the two different models, without and with the stretching terms, were calculated using the low and high Capillary numbers from the data set. The models are dependent on the film thickness of $\mathrm{A}$ which is dependent on the Capillary number, this differs from the models for round channels where the thin film was assumed to be close to zero. The film in the corners, for fluid A, gets thicker due to the Concuss-Finn criteria and can no longer be assumed to be small. The upper set of data, $\nabla$ and $\square$, includes the interface stretching term, the lower set of data, $\triangle$ and $\bigcirc$, is the predictions without the interface stretching terms. As the slug decreases in length the role of the interface stretching increases, as the slugs get longer the predictions begin to collapse to a single line. This is expected as the drag will begin to dominate the system as the slug increases, the drag has a dependence on length.

For prewet square channels, fluid is retained in the corners of channel. This can be seen in Figure 5.3(b). A result of the spontaneous nature of the corner flow, the 
slug of ethylene glycol will begin to traverse the channel without the introduction of the second fluid. The slug will come to rest close to the middle of the channel when the pressure from the corners are balanced on both sides of the menisci. To produce a bislug the force of gravity is used to push the slug to one end, silicone can be drawn into the glass through capillary forces. This may be a possible reason for the faster response in the microchannels and some inconsistencies in the data. A channel that was inverted longer during prewetting may produce a faster response than a channel that was inverted for a shorter period of time because of the amount of fluid that may have drained from the corners. When the tube is realligned horizontally, the pressure imbalance in the corners may help drive the flow for faster response. A bubble rising in a vertically aligned square channel, with stagnant fluid, showed the flow in the corners was significantly faster than the flow in the film when the $C a<10^{-4}[19]$. The Ca numbers for these channels are a magnitude larger than the Ca studied with the bubble flow, but the research shows the corners drain faster than the film. The pressure potential from the corners plays a significant role in bislug for in square microchannels. 


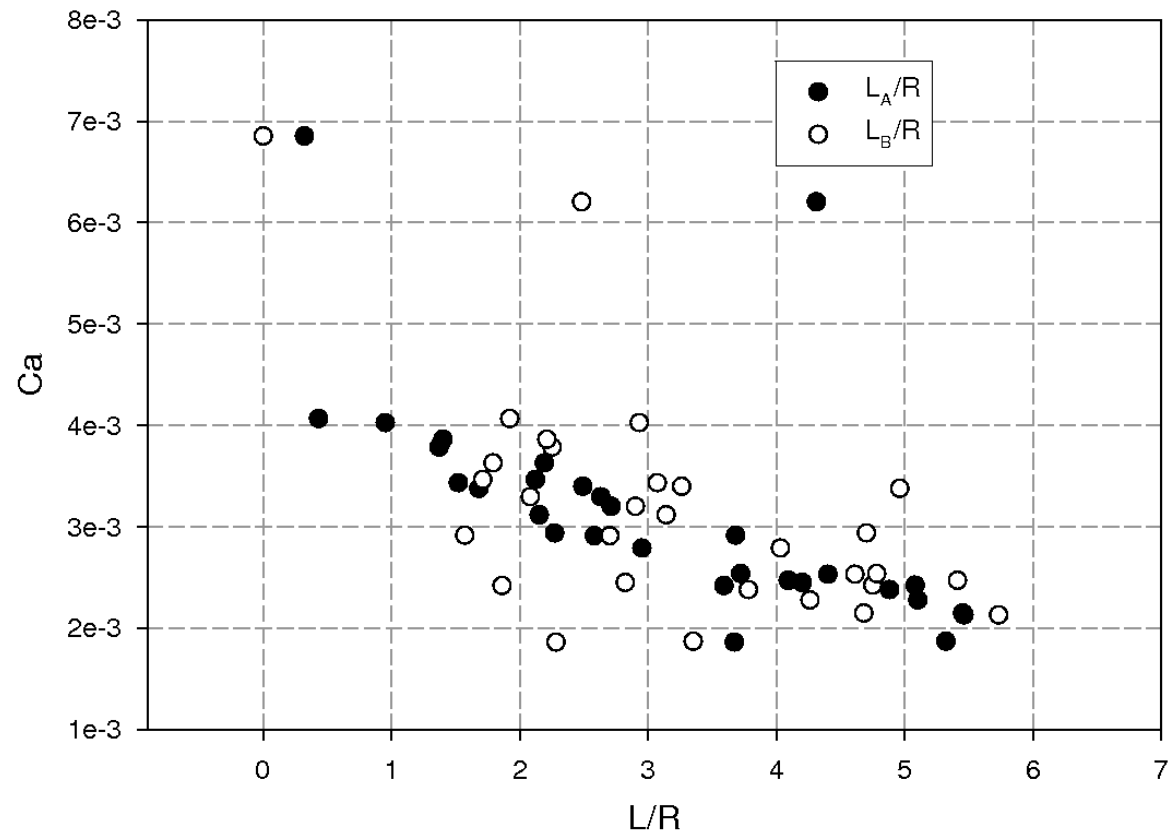

Figure 5.5. Ca number of ethylene glycol based on the front meniscus and the ratio of the slug length to the radius.

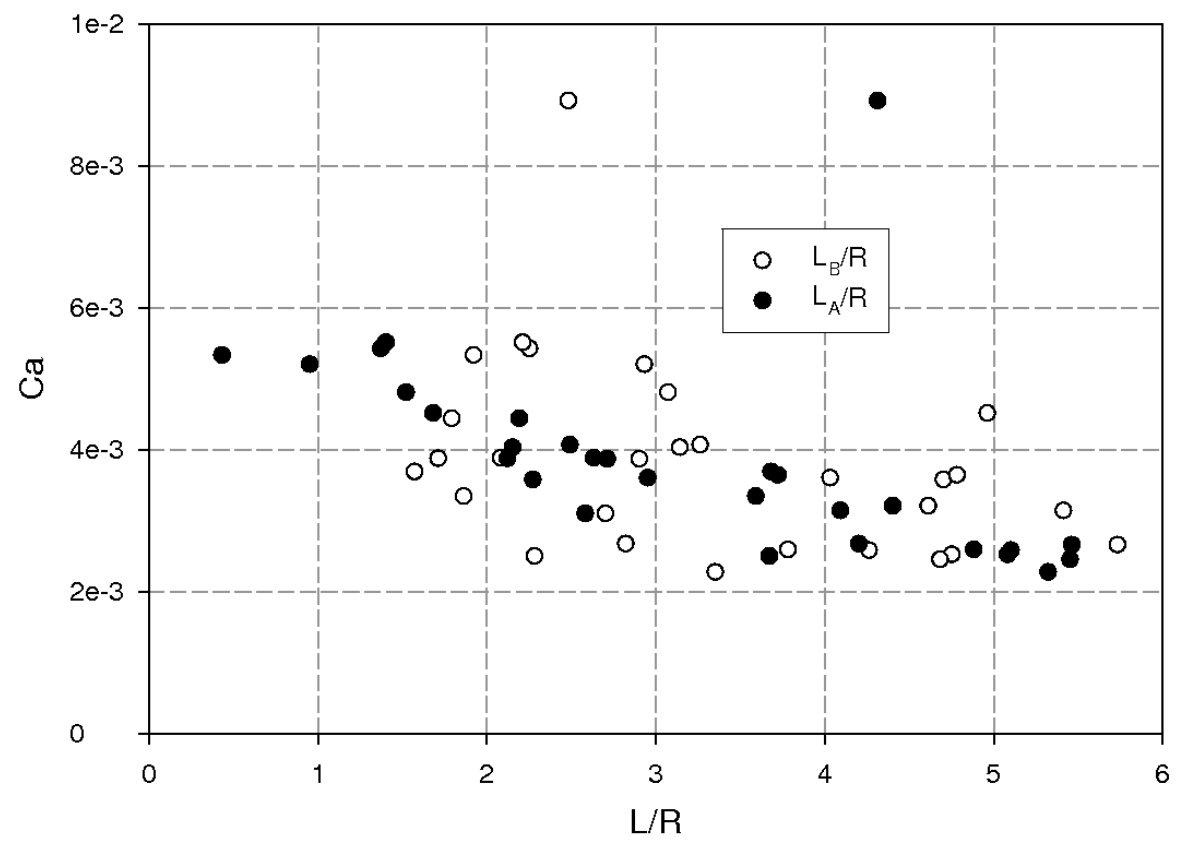

Figure 5.6. Ca number of ethylene glycol based on the rear meniscus and the ratio of the slug length to the radius. 


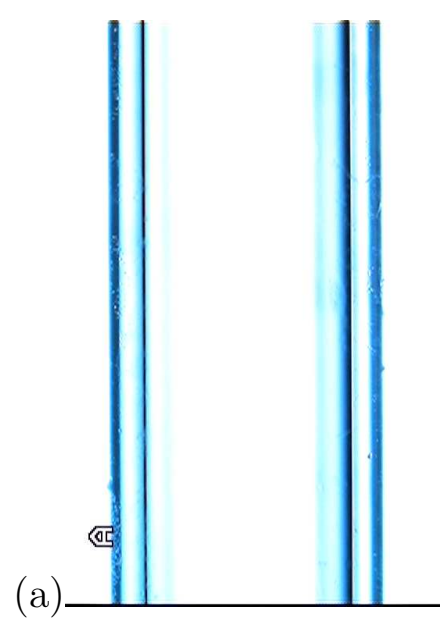

(b)
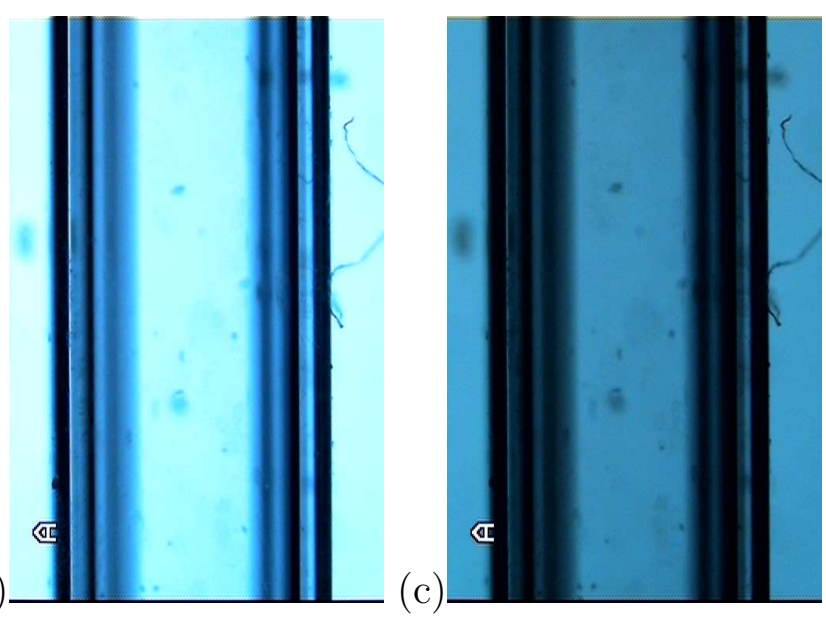

Figure 5.7. Images used to estimated the film thickness on the walls of a square microchannel. (a) Used for scaling images. (b) and (c) Used for measuring the amount of fluid retained in the corner and the width of the flat region of the prewet film. 


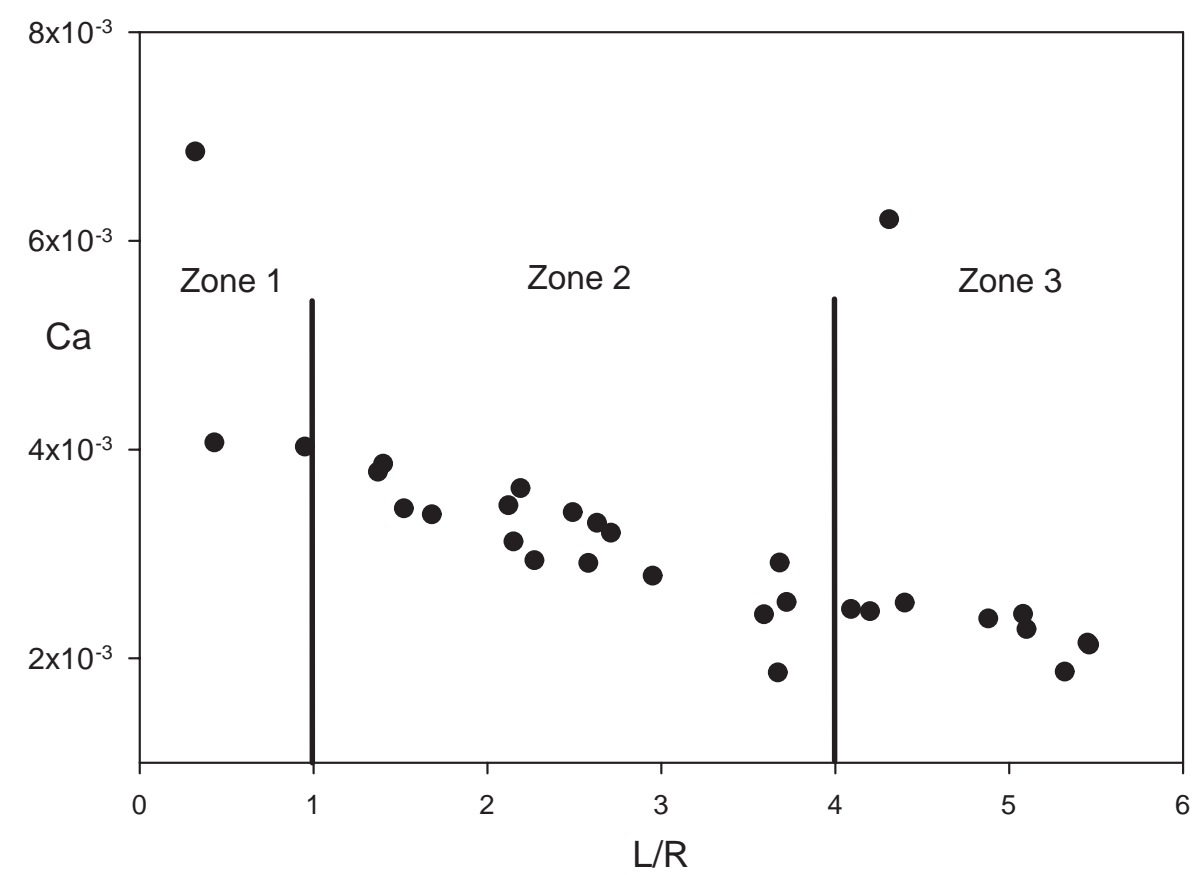

Figure 5.8. The different energy dissipation zones present in bislug system based on the data collected from the front meniscus.

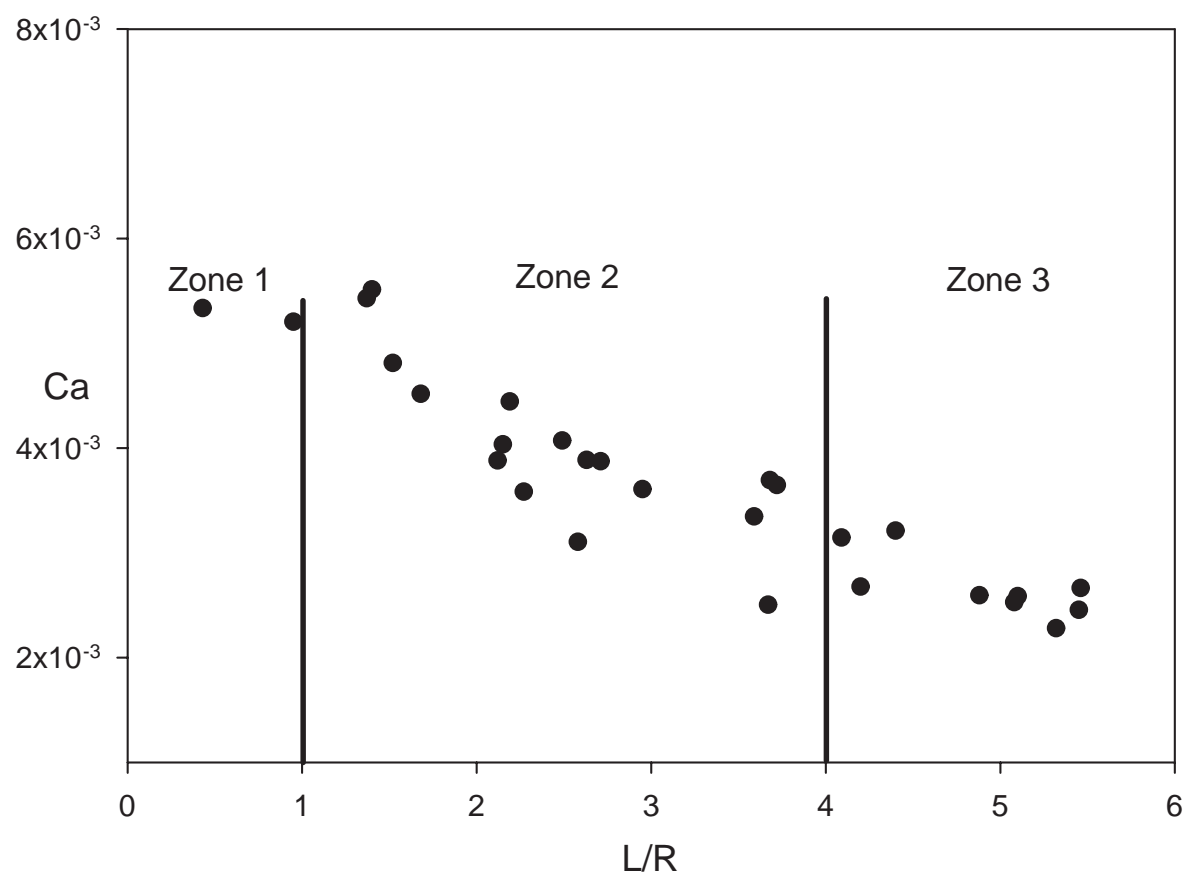

Figure 5.9. The different energy dissipation zones present in bislug system based on the data collected from the rear meniscus. 

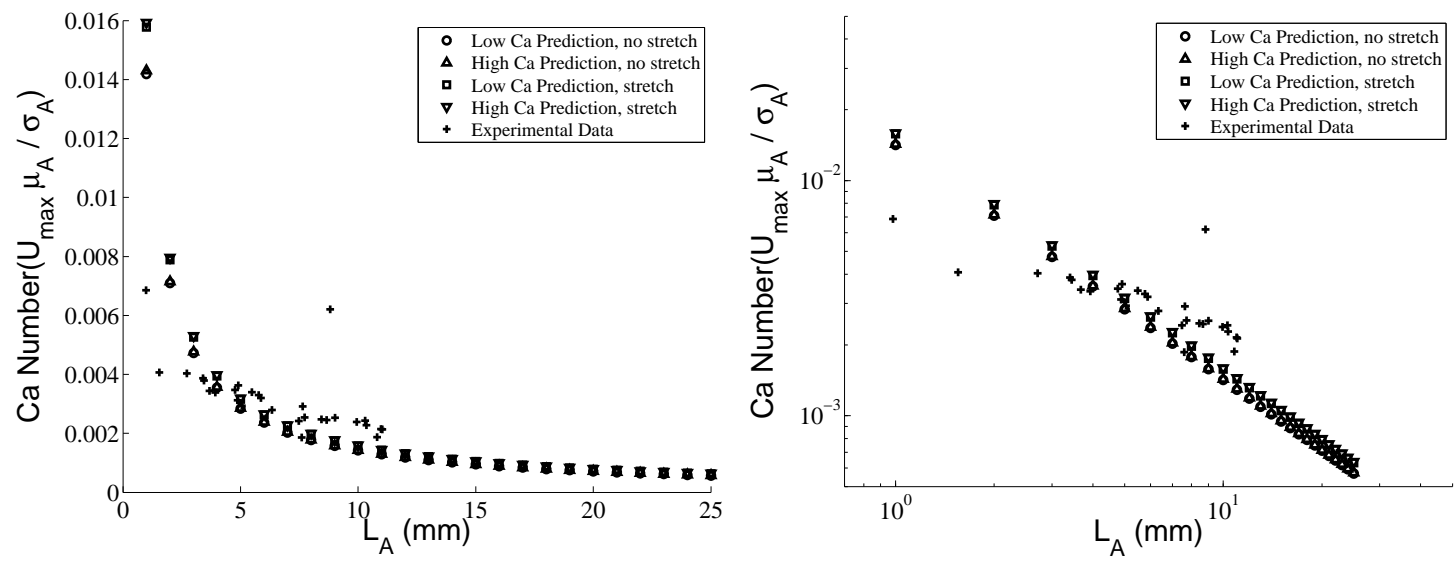

Figure 5.10. Comparison of the experimental data to the developed model with and without stretch. (a)left, data from the front of the meniscus (b)right, the same data plotted on a log-log graph.
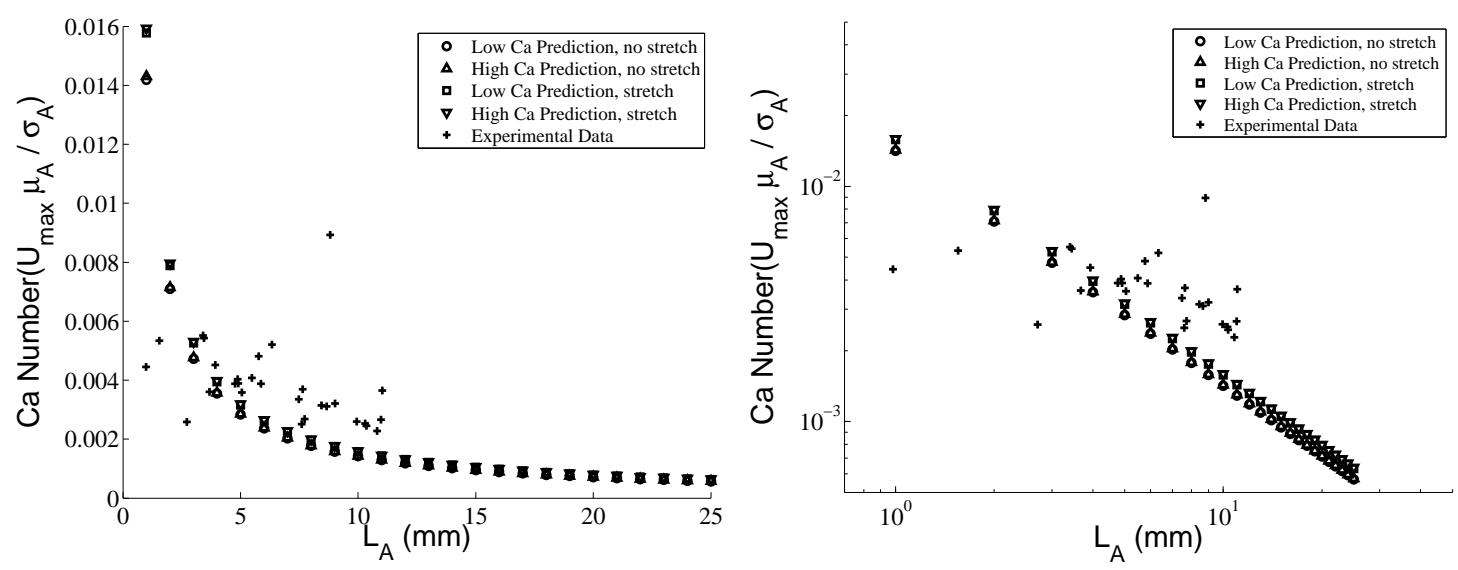

Figure 5.11. Comparison of the experimental data to the developed model with and without stretch. (a)left, data from the rear of the meniscus (b)right, the same data plotted on a log-log graph. 


\section{Chapter 6}

\section{Force Terms}

The derivation was performed using a force balance between the motive force the drag force and the interface stretching. There are other force terms that reduce the overall speed of the bislug. These include the hydrodynamic interaction between menisci of short slugs and the energy dissipation at the contact line.

\subsection{Hydrodynamic Interaction}

Hydrodynamic interactions occur when a slug is small, less than two diameters in length. A short slug has undeveloped streamlines and the menisci interfere with the velocity distribution[18]. Slow moving slugs, $\mathrm{Ca}^{1 / 2}<.5$ produce vortices inside of the slugs, the Ca numbers for bislug flow is less than .5[18]. This interaction shifts streamlines and dissipates energy in the form of viscous heating. This dissipation mechanism can be seen in the experimental data for the square channels, Figures 5.8 and 5.9. The bislug increases in velocity as the slugs reduce in length up to some critical length. Below that length the velocity of the system remains a constant velocity even though the viscous drag is decreasing. The mensisci interaction dominate the system at the small lengths.

\subsection{Contact Line}

Energy dissipation associated with the moving contact line was not included in the force balance due the complexity of the interactions at the interface. A problem that arises at the contact line is a singularity that is formed when assuming a no-slip boundary condition, the shear stress goes to infinity. Dussan (1976) removed the singularity by assuming a slip boundary condition, to include this dissipation mechanism assuming the no-slip condition at the wall may no longer be valid approach. Ludviksson (1968) removed the singularity by assuming the channel was precoated by an advancing film ahead of the contact line. Using either assumption removes the physics from the local area and introduces new values that need to be found numerically or experimentally.

Contact line dissipation has been modelled using a dynamic contact angle ap- 
proach. As a fluid moves across an interface, solid or fluid, the viscous stresses at the contact line distort the interface. This distortion changes the contact angles for the system, the front increases and the rear decreases. In principle the local energy dissipation is captured by the dynamic contact angle, $\theta_{d}$. Cox (1986) provides a model for the dynamic contact angle for a liquid displacing a gas. Fermigier et al. (1988) verified that the model could also be used for a liquid-liquid pair that had a small viscosity ratio. Van Der Zanden et al. (1994) provides a model for moving liquidliquid contact lines with small viscosity ratios. Their work is limited by a critical $\mathrm{Ca}$ number. Though the physics at the contact line can be represented by the dynamic contact angle, it does not capture the true dissipation.

\subsection{Non-Poiseuille flow}

The flow profile for the slugs were assumed to be Hagen-Poiseuille but there is evidence that the stream lines are not parabolic. Taylor (1961) presented sketches of possible velocity profiles in pressure driven bubble-train flow. When the $C a^{1 / 2}>.5$ the velocity streamlines bipass the bubble completely. If the $\mathrm{Ca}^{1 / 2}<.5$ recirculation vortices form in the slug. The velocity profiles are no longer classic Hagen-Poisseuille flow but are now treadlike. Prothero et al( 1961) injected ink into slugs of liquid and noted that the ink travel the length of the slug after the slug had traveled two lengths. This was later verified by Thulasidas et al (1997) who noted the same time interval for a particle to travel one length of the slug. Also, micro-PIV images were included that proved the flow patterns predicted by Taylor (1961) were correct. Dussan et al (1974) showed treadlike flows in a drop rolling along a solid. This same idea can be extrapulated to a drop in a microchannel with substrate on all sides. Bruno [7], using $\mu \mathrm{PIV}$ in square microchannels, reports the flow to be treadlike and not classic HagenPoseuille flow as previously suggested, but the experimental results are preliminary. Further investigation is needed for developement of a flow profile resembling those found in the slugs.

The treadlike flow profile my be the result of the conservation of mass in a 3$\mathrm{D}$ configuration. The centerline velocity has to be faster than the menisci velocity to maintain the conservation of mass on the surface. As a material particle moves from the center of the menisci to the wall, as illustrated in Figure 6.1, it moves over a larger surface area at the channel wall that at the centerline. The surface of the meniscus, from the centerline to the wall, is moving at the same velocity in the direction parallel with the channel. The material particle is traveling in both the direction of the channel and the following the path of the meniscus. This increased path requires a faster centerline velocity. An increased centerline velocity would setup a recirculation in the slug of the fluid producing the observed treadlike profile.

\subsection{Corner Flow}

The velocity profile for the thin film in the square channels were assumed to be Couette or linear. Weislogel(1996) provides data and equations that quantify the 


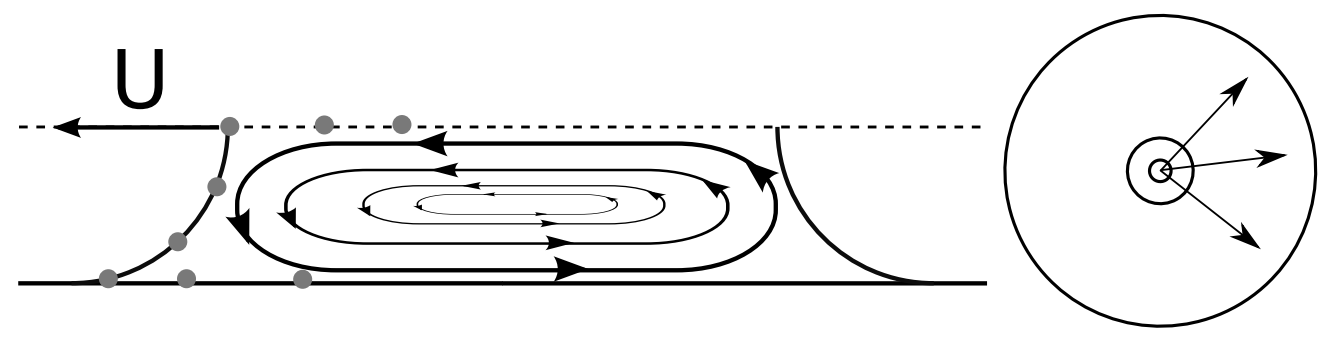

Figure 6.1. Recirculation caused by faster centerline velocity relative to the menisci, faster velocity is required to satisfy the conservation of mass for the system.

amount of liquid being pumped into the corners from the meniscus. Pumping from the mensicus was verified by the use of a laser scanning confocal microscope at Sandia National Labs, Appendix A. Figure A.1 shows excited particles in a slug of ethylene glycol, as the slug remains stationary, fluid is pumped from the meniscus to the corner. For the leading meniscus, fluid is being pumped ahead of the mensicus as it travels down the channel. This may increase the velocity of the overall system. When the bislug was produced the channel was first dipped in ethylene glycol and inverted to coat the channel. The amount of time the channel was inverted may have added an uncertainty to the problem. The amount of fluid drained from the corners may have changed from test run to test run, see Chapter 5 . This additional flowrate, along with the thicker film in the corners, may be the reason for the faster flows in microchannels that have corners and satisfy the Concus-Finn criteria. The derivation should be redone with the inclusion of the Hagen-Poiseuille flow profile in the film. 


\section{Chapter 7}

\section{Conclusion}

Spontaneous bislug flow can occur in microchannels of circular and non-circular crosssections. Microchannels with corners are subject to the Concus-Finn criteria. Fluid retention in the corners reduces the energy dissipation associated with the moving contact line and shear stress. The thicker fluid in the corners act like lubricating "rails" for the fluid to travel along.

A model has been developed that uses a balance of the pressure difference over of the menisci with the drag force and the force associated with the stretching of the interfaces at the menisici. Values for the experimental data are lower than those calculated using the model. The difference between the model and the data can be attributed to the energy dissipation mechanisms absent from the model and the limited slug lengths obtainable from experiments.

Bislug flow is a constant pressure system which will provide a repeatable test condition. The four major energy dissipation mechanisms can be studied using this technique. Short slugs will provide information on menisci interation, long slugs will verify models for shear stress at the wall, and contact line dissipation can be studied from the transition from long to short slug lengths. The summation of the interface stretching term for a bislug system may produce a negative energy dissipation or a net energy gain to the system, as seen in these experiments. If the surface tension of the trailing menisci have a total value less than the leading meniscus a net energy increase occurs. The prewet channel has stored energy in the stretched precursor film. As the bislug travels down the channel the leading slug relaxes the interface absorbing energy into the system. The amount of energy required to stretch the trailing liquids is less that the amount gained resulting in positive energy gain for the system. This may be the reason for the faster response in prewet channels, in addition to the reduction of the contact line dissipation. It has been seen experimentally that a slug of fluid will spontaneously travel down a prewet channel without the introduction of a trailing fluid. The slug may be balancing the system energy by reducing the stored energy in the advancing film and adding energy to the trailing film. 


\section{Bibliography}

[1] Energy Demands on Water Resources., report to congress on the interdependency of energy and water, 2006.

[2] Allen, Jeffrey S. Two-Phase Flow in Small Channels and the Implications for PEM Fuel Cell Performance The Electrochemical Societey, 3(1):1997-1206, 2006.

[3] Bai, Runyan, Chen, Kangping, and Joseph, D. D. Lubricated Pipelining: Stability of Core-Annular Flow. Part 5. Experiments and Comparison With Theory. J. Fluid Mech, 240:97-132, 1992.

[4] Bico, J. and Quere, D. Liquid Trains in a Tube. Europhysics Letters, 51:546550, 2000 .

[5] Bico, J. and Quere, D. Self-Propelling Slugs. J. Fluid Mech., 467:101-127, 2002.

[6] Bretherton, F. P. The Motion of Long Bubbles in Tubes. Journal of Fluid Mechanics, 10(2):166-168, 1961.

[7] Bradford, Bruno, Martel, Joseph and Black, Kelly. A Preliminary PIV and Analytical Investigation of Wall Shear in Micro Channel Slug Flow. In ASME International Mechanical Engineering Congress and Exposition, Seattle, Washington, USA, 2007. Asme.

[8] Concus, Paul and Finn, Robert On the Behavior of a Capillary Surface in a Wedge. Applied Mathematical Sciences, 63:292-299, 1969.

[9] Dussan V., E. B. The Moving Contact Line: Slip Boundary Condition. J. Fluid Mech, 77(4):665-684, 1976.

[10] Fairbrother, F. and Stubbes, A. E. Studies in Electroendosmosis-VI. The "Bubble Tube" Method of Measurement. Journal of Chemical Society, 1:527-529, 1935.

[11] Fermigier, M. and Jenffer, P. Dynamics of a Liquid-Liquid Interface in a Capillary. Ann. Phys., 13, 1998. 
[12] Wright, Ted and Klimek, Robert. http://microgravity.grc.nasa.gov/spotlight/, 2005.

[13] Kolb, W. Blake and Cerro, Ramon L. Coating the Inside of a Capillary of Square Cross Section Chemical Engineering Science, 46(9):2181-2195, 1991.

[14] Ludviksson, V. and Lightfoot, E. N Deformation of Advancing Menisci. AIChE journal, 14, 1968.

[15] Middleman, Stanley Modeling Axisymmetric Flows: Dynamics, Films, Jets, and Drops. Academic Press, San Diego, 1995.

[16] Prothero, J. and Burton, J. C. The Physics of Blood Flow in Capillaries I. The Nature of the Motion. Biophysical Journal, 1:565-579, 1961.

[17] Taylor, G. I. Deposition of a Viscous Fluid on the Wall of a Tube. J. Fluid Mech, 10:161-165, 1961.

[18] Abraham, M. A., Thulasidas, T.C. and Cerro, R. L. Flow Patterns in Liquid Slugs During Bubble Train Flow Inside Capillaries. Chemical Engineering Science, 52(17):2947-2962, 1997.

[19] Abraham, M. A., Cerro, R. L. and Thulasidas, T. C. Dispersion During BubbleTrain Flow in Capillaries. Chemical Engineering Science, 54:61-76, 1999.

[20] Van Der Zanden, A. J. J., and Chesters, A. K. An Approximate Solution of the Hydrodynamic Problem Associated with Moving Liquid-Liquid Contact Lines. Int. J. Multiphase Flow, 20(4):789-798, 1994.

[21] Washburn, Edward W. The Dynamics Of Capillary Flow. The Physical Review, 17(3):273-283, 1921.

[22] Weislogel, Mark M. Capillary Flow in an Interior Corner, NASA Technical Memorandum-107364, 1996.

[23] White, Frank M. Viscous Fluid Flow. McGraw-Hill Science/Engineering/Math, Boston, 2nd edition, 1991. 


\section{Appendix A}

\section{Confocal Imaging of Corner Flow}

As previously discussed, the response time of the bislug is an important factor when designing a micropump. Microchannels of square cross-section produce a faster bislug flow than microchannels of round cross-section. To properly predict the reponse of the system, an understanding of the energy dissipation is needed. Visualization of the flow fields in a slug is hindered by the meniscus. The meniscus acts like a lens diverting light as it passes through. The menisci curvature prevents the viewing of the center line of the slug near the menisci. This region is of interest for studying dissipations caused by both the contact line and menisci interation. To visualize flow in this area a confocal microscope may be used.

The LSCM uses a laser to excite a sheet of fluorescent particles. The light is collected through a pinhole that scans across a CCD sensor. The system at Sandia was capable of exciting and recording at two different light frequencies. Fluorescent coated polystyrene beads with a diameter of $15 \mu \mathrm{m}$ (Duke Scientific) and excitation frequencies of $468 \mathrm{~nm}$ and $542 \mathrm{~nm}$, and emitting frequences of $508 \mathrm{~nm}$ and $612 \mathrm{~nm}$, were mixed in the ethylene glycol and silicone, respectively. Larger beads were used to decrease the system's dependency on lighting. As the beads decrease in diameter the amount of light emitted also decreases. Using larger beads avoided the potential problems associated with decreased light emittance. An ultrasonic water bath was used to break up the mircobeads to produce a uniform dispersion; clumps of beads were still present after treatment and can be seen in Figure A.1. 100 cst silicone oil was used in place of the of 1 cst silicone oil to slow down the bislug, the LSCM has frame rate on the 0 of $1 \mathrm{fps}$ which is too slow for the faster moving slugs.

Smaller square microchannels were used due to the optical limitations of the microscope. The depth of the $1 \mathrm{~mm}$ microchannels were outside the range of the microscope, so 500 and $300 \mu \mathrm{m}$ square channels were used. To control the slug lengths and trigger the flow, gates where made from Poly-DiMethyl Siloxane (PDMS). The primary design had a long central channel for the microchannel to fit into and two smaller channels branching off at the other end. Two syringes containing the working fluids were attached to the channels that branched off while a third needle, attached 


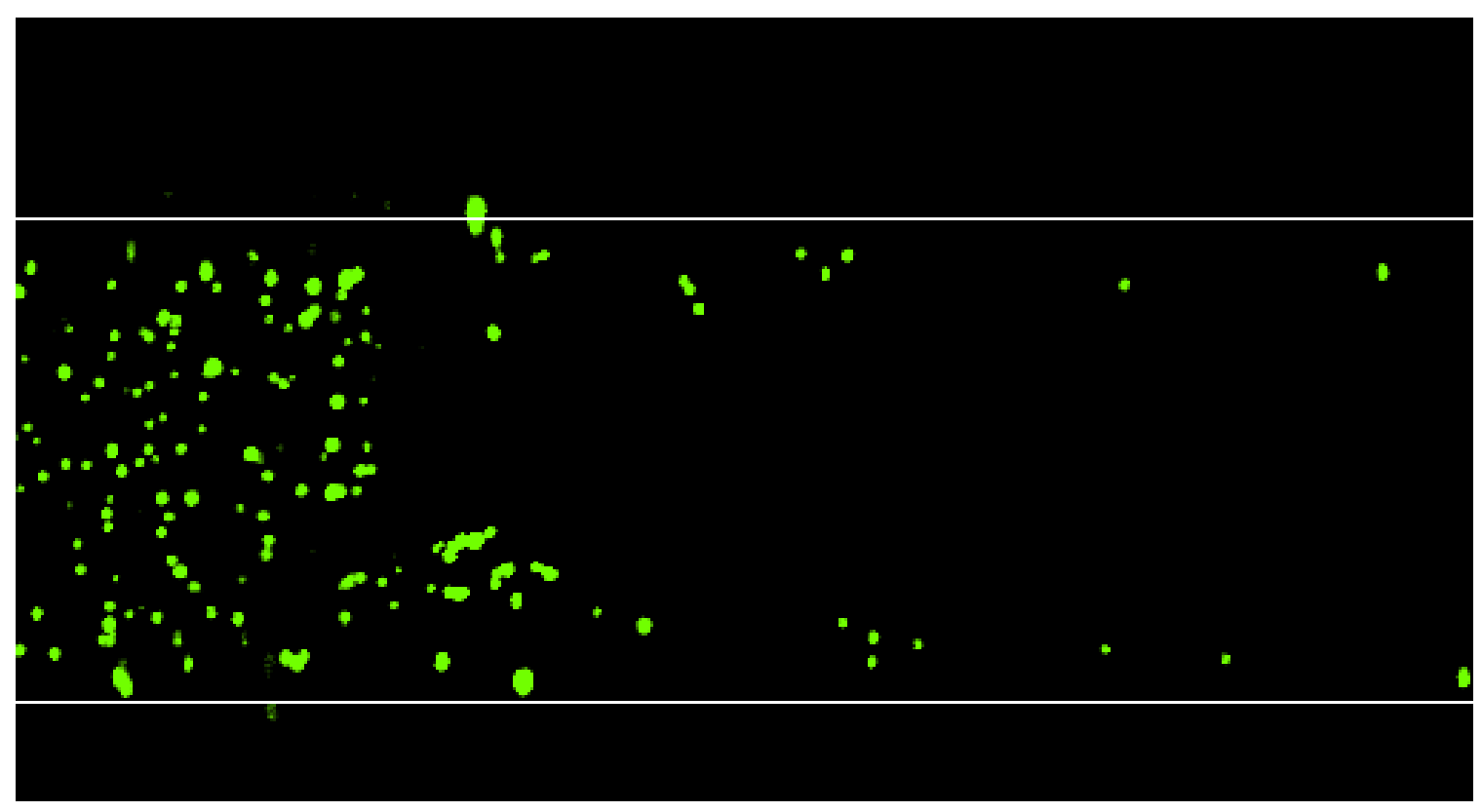

Figure A.1. Corner flow captured using a LSCM.

to a valve which opened to a syringe full of air or atmospheric pressure, was attached to the end of the long channel. The microchannel was prewet before being inserted into the PDMS. The branching channels were filled with fluid and the bislug was formed in the microchannel. The syringe full of air was used to break the bislug free and the system was then opened to atmospheric pressure. This method was only used a couple of times due to problems with the PDMS bonding. The dipping method, described earlier, was used instead.

Due to time contraints and system properties the data was recorded for visual purposes only, the slow frame rate and the linescanning image capture method produced images that were skewed. Images of the spontaneous flow in the corners were recorded. Figure A.1 shows a single frame. The flow was slower and could be accurately captured by the LSCM. Fluid flowed from the center of the meniscus to the corners. This may explain why the slug of ethylene glycol would traverse towards the middle of the channel. The slug would move as the fluid was being pulled from the center to the corners. Another issue with the LSCM is evident in Figure A.1 at the top of the channel. The gain for the camera had to be adjusted so beads were large enough to be seen but not too large that the beads appeared outside of the channel, which is what happened in this case. To prevent this a trial and error method was used to adjust the size of the beads for practicle recording. A LSCM is capable of capturing the flow near a moving bislug meniscus but the slow frame rate produces images that are not quantifiable. The images captured were skewed and had too large of a distance traveled between particles for tracking accurately. 


\section{Appendix B}

\section{Round Derivation}

\section{B.1 Derivation of Force Terms}

Three terms are included in the force balance; the motive force, the drag force and the force associated with the stretching of the interface at the menisci.

$$
F_{\text {motive }}=F_{\text {drag }}+F_{\text {stretch }}
$$

\section{B.1.1 Pressure Force}

The motive force is caused by a pressure imbalance across the three menisci.

$$
F_{\text {motive }}=\Delta P A_{\text {cross }}=\frac{2\left(\sigma_{A}-\sigma_{B}-\sigma_{A B}\right)}{R}\left(\pi R^{2}\right)
$$

Where $A_{\text {cross }}$ is the projected cross-sectional area of the menisci.

\section{B.1.2 Interface Stretching}

The stretching force was defined as the surface tension $\sigma$ multiplied by the coated perimeter $P_{e}$. This occurs across each of three menisci in the bislug system.

$$
F_{\text {stretch }}=\sigma P_{e}
$$

As the bislug flows in a prewet round channel there is a continuous destruction of the interface at $\mathrm{A}$ and the creation of one at $\mathrm{AB}$ and $\mathrm{B}$. The creation at $\mathrm{AB}$ is done over the thin film $h_{A}$.

$$
\begin{aligned}
& \text { Destruction of A-air interface : } \quad-\sigma_{A} 2 \pi\left(R-h_{A}\right) \\
& \text { Creation of A-B interface : } \sigma_{A B} 2 \pi\left(R-h_{A}\right) \\
& \text { Creation of B-air interface : } \sigma_{B} 2 \pi\left(R-h_{A}-h_{B}\right) \\
& F_{\text {stretch }}=2 \pi R\left[-\sigma_{A}\left(1-\frac{h_{A}}{R}\right)+\sigma_{A B}\left(1-\frac{h_{A}}{R}\right)+\sigma_{B}\left(1-\frac{h_{A}}{R}-\frac{h_{B}}{R}\right)\right] \\
& =2 \pi R\left[\left(1-\frac{h_{A}}{R}\right)\left(\sigma_{A B}+\sigma_{B}-\sigma_{A}\right)-\sigma_{B} \frac{h_{B}}{R}\right]
\end{aligned}
$$




\section{B.1.3 Drag Force}

The drag force is divided into 3 terms, the core of slug $\mathrm{A}$, the thin film of $\mathrm{A}$ beneath core $\mathrm{B}$, and the core of $\mathrm{B}$.

$$
F=F_{\text {core } A}+F_{\text {film } A}+F_{\text {core } B}
$$

\section{B.1.3.1 Core A}

Core A is modeled using the lubrication approximation.

$$
0=-\frac{\partial P}{\partial x}+\frac{\mu}{r} \frac{\partial}{\partial r}\left(r \frac{\partial u}{\partial r}\right)
$$

The pressure potential, $\partial P / \partial x$, is treated as a constant, $K$, which physically represents the pressure imbalance across the slug divided by the bislug length.

$$
\frac{K}{\mu}=\frac{1}{r} \frac{\partial}{\partial r}\left(r \frac{\partial u}{\partial r}\right)
$$

Integrating twice to find the velocity and applying the no-slip condition at the wall and a symmetry condition at the centerline:

$$
u=U_{\max }\left[1-\left(\frac{r}{R}\right)^{2}\right]
$$

where

$$
U_{\max }=-\frac{K R^{2}}{4 \mu}
$$

The shear stress at the wall is:

$$
\tau_{w_{A}}=\left.\mu \frac{\partial u}{\partial r}\right|_{r=R}=-\frac{2 \mu_{A} U}{R}
$$

\section{B.1.3.2 Film A}

Film A can be modeled as Couette flow. There is a no-slip condition on the wall and a moving boundary driven by Core B. Starting with lubrication approximation B.7, with $\partial P / \partial x$ being equal to zero and the $\mu / r$ being constants, the working form becomes:

$$
0=\frac{\partial}{\partial r}\left(r \frac{\partial u}{\partial r}\right)
$$

Integrating twice

$$
\begin{aligned}
& 0=\int \frac{\partial}{\partial r}\left(r \frac{\partial u}{\partial r}\right) \\
& u=C_{0} \ln r+C_{1}
\end{aligned}
$$


Applying the boundary conditions:

$$
\begin{aligned}
& \text { at } r=R, u=0 \\
& \text { at } r=R-h_{A}, u=U_{A B}
\end{aligned}
$$

Results in:

$$
\begin{aligned}
u & =C_{0} \ln \left(\frac{r}{R}\right) \\
C_{0} & =\frac{U_{A B}}{\ln \left(R-h_{a}\right)} \\
u & =U_{A B} \frac{\ln \left(\frac{R}{r}\right)}{\ln \left(\frac{R}{R-h_{A}}\right)}
\end{aligned}
$$

The final form B.15 matches the previously derived solution for a set of concentric cyclinders with the center cylinder in motion [23]. The shear stress for the film can be found be taking the derivative of the velocity w.r.t the radius of the channel.

$$
\tau=-\frac{\mu_{A} U_{A B}}{r \ln \left(\frac{R}{R-h_{A}}\right)}
$$

\section{B.1.3.3 Core B}

Core $\mathrm{B}$ is modeled using the lubrication approximation in the same fashion as Core A B.7.

$$
u=\frac{K}{4 \mu} r^{2}+F(x) \ln (r)+g(x)
$$

Applying the boundary condition at $r=0$

$$
\begin{aligned}
r & =0, \text { symmetry } \\
0 & =\left.\frac{\partial u}{\partial r}\right|_{r=0} \\
& =\frac{2 K r}{4 \mu}+\left.F^{\prime}(x) \frac{1}{r}\right|_{r=0} \\
F^{\prime}(x) & =0 \rightarrow F(x)=0 \\
u & =U_{\max } \\
g(x) & =U_{\max } \\
u & =\frac{k}{4 \mu_{B}} r^{2}+U_{\max }
\end{aligned}
$$


Applying boundary condition at $r=R-h_{A}$

$$
\begin{aligned}
u & =U_{A B}, \text { at } r=R-h_{A} \\
U_{A B} & =\frac{k}{4 \mu_{B}}\left(R-h_{A}\right)^{2}+U_{\max } \\
U_{\max } & =U_{A B}-\frac{k}{4 \mu_{B}}(R-h o)^{2}
\end{aligned}
$$

Solving for the velocity in terms of $U_{A B}$, combining equations B.18 and B.19:

$$
\begin{aligned}
u & =-\frac{k}{4 \mu_{B}}\left(R-h_{A}\right)^{2}\left(1-\frac{r^{2}}{\left(R-h_{A}\right)^{2}}\right) \\
u & =\left(U_{\max }-U_{A B}\right)\left(1-\frac{r^{2}}{\left(R-h_{A}\right)^{2}}\right)+U_{A B}
\end{aligned}
$$

The shear stress is:

$$
\tau=-2 \mu_{B}\left(U_{\max }-U_{A B}\right) \frac{r}{\left(R-h_{A}\right)^{2}}
$$

\section{B.1.4 Final Velocity Equations}

$U_{A B}$ can be found from the boundary condition present at the film. The velocities and shear stresses are equal for both the film and the Core by assigning a zero slip boundary condition at a local point.

$$
\begin{aligned}
& u_{\text {filmA }}=u_{\text {CoreB }}=U_{A B} \\
& \left.\tau_{\text {filmA }}\right|_{r=R-h_{A}}=\left.\tau_{\text {CoreB } B}\right|_{r=R-h_{A}}
\end{aligned}
$$

This results in the solutions for film A at the boundary being:

$$
\begin{aligned}
u_{f i l m A} & =U_{A B} \\
\tau_{f i l m A} & =-\frac{\mu_{A} U_{A B}}{\left(R-h_{A}\right) \ln \left(\frac{R}{R-h_{A}}\right)}
\end{aligned}
$$

Solutions for Core B are:

$$
\begin{aligned}
u_{\text {CoreB }} & =U_{A B} \\
\tau_{\text {Core } B} & =-\frac{2 \mu_{B}\left(U_{\max }-U_{A B}\right)}{R-h_{A}}
\end{aligned}
$$

Setting equations B.23 and B.24 equal, $U_{A B}$ can be solved.

$$
U_{A B}=\frac{U_{\max }}{1+\frac{1}{2} \frac{\mu_{A}}{\mu_{B}} \frac{1}{\ln \left(\frac{R}{R-h_{A}}\right)}}
$$


The final form for the velocities and shear stress are:

$$
\begin{aligned}
& u_{f i l m A}=U_{\max } \frac{\ln \left(\frac{R}{r}\right)}{\ln \left(\frac{R}{R-h_{A}}\right)+\frac{1}{2} \frac{\mu_{A}}{\mu_{B}}} \\
& \tau_{f i l m A}=-\frac{\mu_{A} U_{\max }}{r\left(\ln \left(\frac{R}{R-h_{A}}\right)+\frac{1}{2} \frac{\mu_{A}}{\mu_{B}}\right)}
\end{aligned}
$$

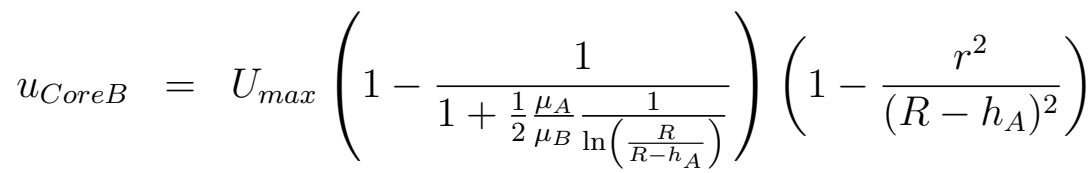

$$
\begin{aligned}
& \tau_{\text {Core } B}=-2 \mu_{B} U_{\max } \frac{r}{\left(R-h_{A}\right)^{2}}\left(1-\frac{1}{1+\frac{1}{2} \frac{\mu_{A}}{\mu_{B}} \frac{1}{\ln \left(\frac{R}{\left(R-h_{A}\right)^{2}}\right)}}\right)
\end{aligned}
$$

\section{B.1.4.1 Total Drag}

When completing the final drag term, $h_{A}$ and $h_{B}$ are small and are ignored.

Fluid A:

$$
\begin{aligned}
& \operatorname{Core}\left(L_{A}\right): \tau_{w}=-\frac{2 \mu_{A} U_{\max }}{R} \\
& \operatorname{Film}\left(L_{B}\right): \tau_{w}=-\frac{\mu_{A} U_{\max }}{R\left(\ln \left(\frac{R}{R-h_{A}}\right)+\frac{1}{2} \frac{\mu_{A}}{\mu_{B}}\right)}
\end{aligned}
$$

Fluid B:

$$
\operatorname{Core}\left(L_{A B}\right) \tau_{w}=-\frac{2 \mu_{B} U_{\max }}{R-h_{A}}\left(1-\frac{1}{1+\frac{1}{2} \frac{\mu_{A}}{\mu_{B}} \frac{1}{\ln \left(\frac{R}{\left(R-h_{A}\right)}\right)}}\right)
$$

The total drag force is the sum of the drag forces from fluid A and fluid B.

$$
\begin{aligned}
& F_{\text {drag }}=\tau_{w_{A}} A_{\text {Core }_{A}}+\tau_{w \text { Film }} A_{\text {Film } A}+\tau_{w_{B}} A_{\text {Core } B} \\
& =\tau_{w \text { Film }}\left(2 \pi R L_{A}\right)+\tau_{w \text { Film }}\left(2 \pi R L_{B}\right)+\tau_{w B}\left(2 \pi\left(R-h_{A}\right) L_{B}\right) \\
& =-\mu_{A} U_{\max }(2 \pi)\left[2\left(L_{A}\right)+\frac{\left(L_{B}\right)}{\ln \left(\frac{R}{R-h_{A}}\right)+\frac{1}{2} \frac{\mu_{A}}{\mu_{B}}}\right.
\end{aligned}
$$

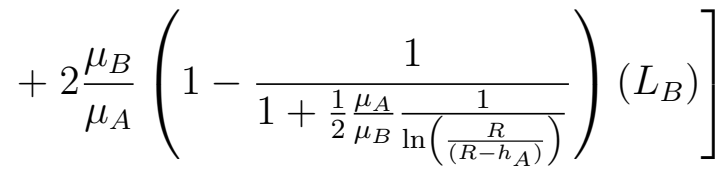


Substituting $\delta$ for $h_{A} / R$ and simplifying yields,

$$
\begin{aligned}
F_{\text {drag }}= & -4 \pi L_{A} \mu_{A} U_{\max }\left[1+\frac{\mu_{B}}{\mu_{A}} \frac{L_{B}}{L_{A}}\right. \\
& \left.+\frac{L_{B}}{L_{A}} \frac{1}{\ln \left(\frac{1}{1-\delta}\right)+\frac{1}{2} \frac{\mu_{A}}{\mu_{B}}}\left(\frac{1}{2}-\frac{\mu_{B}}{\mu_{A}} \ln \left(\frac{1}{(1-\delta)}\right)\right)\right]
\end{aligned}
$$

$\delta$ can be found by using the Bretherton relationship [6].

$$
\delta=\frac{h_{A}}{R}=1.34 \mathrm{Ca}^{\frac{2}{3}}
$$

Assuming $\delta$ is small because the Ca number is small. Figures 4.5 show the Couette region is small compared to the rest of the profile. Therefore, $\delta \ll 1$ and all the ln terms go to zero resulting in the final form of the drag.

$$
F_{d r a g}=-4 \pi L_{A} \mu_{A} U_{\max }\left(1+2 \frac{\mu_{B}}{\mu_{A}} \frac{L_{B}}{L_{A}}\right)
$$

for $\mathrm{Ca}<10^{-3}$.

\section{B.1.5 Final Form}

Finally the pressure force is set equal to the drag force and the stretching force.

$$
\begin{aligned}
F_{\text {motive }}= & F_{\text {drag }}+F_{\text {stretch }} \\
\frac{2\left(\sigma_{A}-\sigma_{B}-\sigma_{A B}\right)}{R}\left(\pi R^{2}\right)= & F_{\text {drag }}+\sigma_{A B}\left(1-\frac{h_{A}}{R}\right) 2 \pi R \\
& +\sigma_{B}\left(1-\frac{h_{A}+h_{B}}{R}\right) 2 \pi R-\sigma_{A}\left(1-\frac{h_{A}}{R}\right) 2 \pi R \\
\left(\sigma_{A}-\sigma_{B}-\sigma_{A B}\right)= & \frac{F_{\text {drag }}}{2 \pi R}+\left(\sigma_{B}+\sigma_{A B}-\sigma_{A}\right)\left(1-\frac{h_{A}}{R}\right)-\sigma_{B} \frac{h_{B}}{R} \\
\frac{F_{\mathrm{drag}}}{2 \pi R \sigma_{A}}= & \left(1-\frac{\sigma_{B}+\sigma_{A B}}{\sigma_{A}}\right)\left(2-\frac{h_{A}}{R}\right)+\frac{\sigma_{B}}{\sigma_{A}} \frac{h_{B}}{R}
\end{aligned}
$$

Assume, as previously, that the ratio of film thickness to radius is small, $h_{B} / R$, 
$h_{A} / R \ll 1$.

$$
\begin{aligned}
\frac{F_{\mathrm{drag}}}{2 \pi R \sigma_{A}} & \approx 2\left(1-\frac{\sigma_{B}+\sigma_{A B}}{\sigma_{A}}\right) \\
\frac{F_{\mathrm{drag}}}{4 \pi R \sigma_{A}} & \approx\left(1-\frac{\sigma_{B}+\sigma_{A B}}{\sigma_{A}}\right) \\
\frac{4 \pi L_{A} \mu_{A} U_{\max }\left(1+2 \frac{\mu_{B}}{\mu_{A}} \frac{L_{B}}{L_{A}}\right)}{4 \pi R \sigma_{A}} & \approx\left(1-\frac{\sigma_{B}+\sigma_{A B}}{\sigma_{A}}\right) \\
\frac{\mu_{A} U_{\max }\left(1+2 \frac{\mu_{B}}{\mu_{A}} \frac{L_{B}}{L_{A}}\right)}{\sigma_{A}} & \approx\left(1-\frac{\sigma_{B}+\sigma_{A B}}{\sigma_{A}}\right) \frac{R}{L_{A}}
\end{aligned}
$$

The final form of the model is equation B.36.

$$
C a_{\max } \approx\left[1-\left(\frac{\sigma_{A B}+\sigma_{B}}{\sigma_{A}}\right)\right]\left(\frac{R}{L_{A}}\right)\left(\frac{1}{1+2 \frac{\mu_{B}}{\mu_{A}} \frac{L_{B}}{L_{A}}}\right)
$$

for $\mathrm{Ca}<10^{-3}$.

The final form without the removal of the films:

$$
C a_{\max }=\frac{1}{2} \frac{\left[\frac{\Delta \sigma}{\sigma_{A}}\left(2-H_{A}\right)+\frac{\sigma_{B}}{\sigma_{A}} H_{B}\right]}{\left\{1+\frac{L_{B}}{L_{A}}\left(\frac{\mu_{B}}{\mu_{A}}-\frac{L_{B}}{L_{A}} \frac{\frac{1}{2}+\frac{\mu_{B}}{\mu_{A}} \ln \left(\frac{1}{1-H_{A}}\right)}{\ln \left(\frac{1}{1-H_{A}}\right)+\frac{1}{2} \frac{\mu_{A}}{\mu_{B}}}\right)\right\}}
$$




\section{Appendix C}

\section{Square Derivation}

\section{C.1 Derivation of Force Terms}

Three terms are included in the force balance; the motive force, the drag force and the force associated with the stretching of the interface at the menisci, these are the same force terms used in the round derivation B.1.

$$
F_{\text {motive }}=F_{\text {drag }}+F_{\text {stretch }}
$$

\section{C.1.1 Pressure Force}

The motive force is caused by a pressure imbalance across the three menisci, a spherical cap is used for the shape of the square menisci, this results in the same pressure differential found for the round geometries.

$$
F_{\text {motive }}=\Delta P A_{\text {cross }}=\frac{2\left(\sigma_{A}-\sigma_{B}-\sigma_{A B}\right)}{R}\left(\pi R^{2}\right)
$$

Where $A_{\text {cross }}$ is the projected cross-sectional area of the menisci.

The stretching force was defined as the surface tension $\sigma$ multiplied by the coated perimeter $P_{e}$. This occurs across each of three menisci in the bislug system. To find the perimeter for a square channel with a film, the film was broken in to two parts, the flat film and the corner region 4.6. The transition begins where the radius of the corner has a perpendicular intersection with the wall. The shape of the corner is assumed to be a partial circle $(1 / 8)$ with a radius $R_{c}$. For the leading slug the perimeter is that of a normal square.

$$
F_{\text {stretch }}=\sigma P_{e}
$$

As the bislug flows in a prewet round channel there is a continuous destruction of the interface at $\mathrm{A}$ and the creation of one at $\mathrm{AB}$ and $\mathrm{B}$. The creation at $\mathrm{AB}$ is done over the thin film $h_{A}$.

$$
x_{c}=R-x_{f}=R_{c}+h_{A}
$$


Destruction of A-air interface : $\quad-\sigma_{A} 2 \pi\left(R-h_{A}\right)$

Creation of A-B interface : $\quad \sigma_{A B} 8 R\left[\frac{x_{f}}{R}+\frac{\pi}{4}\left(1-\frac{x_{f}}{R}-\frac{h_{A}}{R}\right)\right]$

Creation of B-air interface : $\sigma_{B} 8 R\left[\frac{x_{f}}{R}+\frac{\pi}{4}\left(1-\frac{x_{f}}{R}-\frac{h_{A}}{R}-\frac{h_{B}}{R}\right)\right]$

$$
\begin{aligned}
F_{\text {stretch }}= & 2 \pi R\left(1-\frac{h_{A}}{R}\right)\left(\sigma_{A B}+\sigma_{B}-\sigma_{A}\right) \\
& +8 x_{f}\left(\sigma_{A B}+\sigma_{B}\right)-2 \pi x_{f}\left(\sigma_{A B}+\sigma_{B}\right)-\sigma_{B} R \pi h_{B} \\
= & 2 \pi R\left[\left(1-\frac{h_{A}}{R}\right)\left(\sigma_{A B}+\sigma_{B}-\sigma_{A}\right)-\frac{x_{f}}{R}\left(\sigma_{A B}+\sigma_{B}\right)-\sigma_{B} \frac{h_{B}}{R}\right] \\
& +8 x_{f}\left(\sigma_{A B}+\sigma_{B}\right)
\end{aligned}
$$

\section{C.1.2 Geometry}

To keep a similar derivation with the round channels a distance from the center of the square channel was used. This is broken into two different sections, geometry for the flat region and corner region.

\section{C.1.2.1 Flat Region}

The distance to the film:

$$
\begin{aligned}
S_{f} & =\sqrt{x^{2}+\left(R-h_{A}\right)^{2}} \\
\sigma_{f}=\frac{S_{f}}{R} & =\sqrt{\zeta^{2}+\left(1-H_{o}\right)^{2}}
\end{aligned}
$$

Where $\zeta$ is the ratio $\mathrm{x} / \mathrm{R}$ and $H_{o}$ is $h_{A} / \mathrm{R}$.

The distance to the wall:

$$
\begin{aligned}
S_{o} & =\sqrt{x^{2}+R^{2}} \\
\sigma_{o}=\frac{S_{o}}{R} & =\sqrt{\zeta^{2}+1^{2}}
\end{aligned}
$$

\section{C.1.2.2 Corner Region}

The distance from the center to the wall is the same for the corner and flat region.

$$
\begin{aligned}
h_{c} & =\sqrt{\left(x-x_{f}\right)^{2}+\left(R_{c}+h_{A}\right)^{2}}-R_{c} \\
h_{c}{ }^{\prime} & =\frac{R_{c}+h_{o}}{R_{c}+h_{c}} h_{c} \\
x^{*} & =x-h_{c} \frac{x-x_{f}}{R c+h_{c}} \\
S_{f} & =\sqrt{x^{* 2}+\left(R-h_{c}^{\prime}\right)^{2}}
\end{aligned}
$$


Non-dimensionalized

$$
\begin{aligned}
& H_{c}=\frac{h_{c}}{R}= \sqrt{\left(\zeta-\zeta_{f}\right)^{2}+\left(C+H_{o}\right)^{2}}-C \\
& H_{c}{ }^{\prime}=\frac{h_{c}{ }^{\prime}}{R}= H_{c} \frac{C+H_{p}}{C+H_{c}} \\
& \zeta_{x}=\frac{x^{*}}{R}= \zeta-H_{c} \frac{\zeta-\zeta_{f}}{C+H_{c}} \\
& \sigma_{f}=\frac{S_{f}}{R}= \sqrt{\zeta_{x}{ }^{2}+\left(1-H_{c}{ }^{2}\right)^{2}} \\
& \text { C.2 Drag }
\end{aligned}
$$

\section{C.2.1 Velocity Derivations}

\section{C.2.1.1 Core A}

Assuming Poisseuille flow in the channel and using $\mathrm{S}$ as a distance function, this allows for the same derivation technique used in the round channels. The distance from the center to the wall and the film increases as zeta increase from the ceter to the wall. Starting with the lubrication approximation, s can be substituded for R, both distance measurements from the center point of the channel.

$$
0=-\frac{\partial P}{\partial z}+\frac{\mu}{s} \frac{\partial}{\partial s}\left(s \frac{\partial u}{\partial s}\right)
$$

Integrating twice w.r.t s,

$$
u=\frac{1}{2} C_{0} s^{2}+C_{1} s+c_{2}
$$

Boundary conditions:

$$
\begin{array}{rll}
u=U_{\max } & @ s=0 & \rightarrow U_{\max }=C_{2} \\
\frac{\partial u}{\partial s}=0 & @ s=0 & \rightarrow C_{1}=0 \\
u=0 & @ s=S_{o} & \rightarrow C_{0}=\frac{-2 U}{U_{\max }}
\end{array}
$$

Applying the boundary conditions results in:

$$
u_{\text {Core } A}=U_{\max }\left[1-\frac{s^{2}}{S_{o}{ }^{2}}\right]
$$

This is the same equation for Hagen-Poiseuille flow in a round channel but with the $\mathrm{s}$ term for the radius.

Shear stress at the wall is found by taking the derivative of the velocity with respect to $\mathrm{s}$ and multiplying by $\mu_{A}$ :

$$
\tau_{w}=\left.\mu_{A} \frac{\partial u}{\partial s}\right|_{s=S_{o}}=\mu_{A} \frac{2 U_{\max }}{S_{o}}
$$




\section{C.2.1.2 Film A}

Finding the velocity profile for the film starts with the lubrication approximation,

$$
0=\frac{\partial}{\partial s}\left(s \frac{\partial u}{\partial s}\right)
$$

Integrating twice

$$
\begin{gathered}
0=\int \frac{\partial}{\partial r}\left(r \frac{\partial u}{\partial r}\right) \\
u=C_{0} \ln s+C_{1} \\
u=0 \quad @ s=S_{o} \rightarrow C_{1}=-C_{0} \ln S_{o} \\
u=U_{A B} \quad @ s=S_{f} \rightarrow C_{0}=\frac{U_{A B}}{\ln \frac{S_{f}}{S_{o}}}
\end{gathered}
$$

results in:

$$
\begin{aligned}
u & =C_{0} \ln \frac{s}{S_{o}} \\
C_{o} & =\frac{U_{A B}}{\ln \frac{S_{f}}{S_{o}}} \\
u & =U_{A B} \frac{\ln \left(\frac{s}{S_{o}}\right)}{\ln \left(\frac{S_{f}}{S_{o}}\right)} \\
\text { or, } u & =U_{A B} \frac{\ln \left(\frac{S_{o}}{s}\right)}{\ln \left(\frac{S_{o}}{S_{f}}\right)}, \text { to match the solution for a round channel }
\end{aligned}
$$

\section{C.2.1.3 Core B}

Starting with the lubrication approximation, same as Core A.

$$
0=-\frac{\partial P}{\partial z}+\frac{\mu}{s} \frac{\partial}{\partial s}\left(s \frac{\partial u}{\partial s}\right)
$$

Integrating twice w.r.t s,

$$
u=\frac{k}{4 \mu_{A}} s^{2}+C_{1} s+C_{2}
$$


$\mathrm{k}=\frac{\partial P}{\partial z}$

Boundary conditions:

$$
\begin{array}{rll}
u=U_{\max } & @ s=o & \rightarrow U_{\max }=C_{2} \\
\frac{\partial u}{\partial s}=0 & @ s=0 & \rightarrow C_{1}=0 \\
u=U_{A B} & @ s=S_{f} & \rightarrow U_{A B}=\frac{k}{4 \mu_{A}} S_{f}^{2}+U_{\max }
\end{array}
$$

The velocity is:

$$
u=\frac{k}{4 \mu_{A}} s^{2}+U_{\max }
$$

\section{C.2.1.4 Final Velocity Forms}

$U_{A B}$ can be found in the same fashion as the round channels. At the trasition between film A and Core B it is assumed to have a no-slip condition. This results in a constact velocity and shear stress at a local point at the transition.

B.C.'s

$$
\begin{aligned}
\left.\tau_{\text {core } B}\right|_{s=S_{f}} & =\left.\tau_{\text {filmA }}\right|_{s=S_{f}} \\
\left.u_{\text {core } B}\right|_{s=S_{f}} & =\left.u_{\text {filmA } A}\right|_{s=S_{f}}=U_{A B}
\end{aligned}
$$

$$
\begin{aligned}
\left.t a u_{\text {core } B}\right|_{s=S_{f}} & =\left.\mu_{B} \frac{\partial u}{\partial s}\right|_{s=S_{f}}=-\mu_{B}\left(U_{\text {max }}-U_{A B}\right) \frac{2}{S_{f}} \\
\left.\tau_{f i l m A}\right|_{s=S_{f}} & =\left.\mu_{A} \frac{\partial u}{\partial s}\right|_{s=S_{f}}=-\mu_{A} \frac{U_{A B}}{S_{f} \ln \frac{S_{o}}{S_{f}}}
\end{aligned}
$$

Combining the shear stress and velocities at the transition results in:

$$
U_{A B}=\frac{U_{\max }}{\left(1+\frac{1}{2} \frac{\mu_{A}}{\mu_{B}} \frac{1}{\ln \frac{S_{o}}{S_{f}}}\right)}
$$

non-dimensional:

$$
\frac{U_{A B}}{U_{\max }}=\frac{1}{\left(1+\frac{1}{2} \frac{\mu_{A}}{\mu_{B}} \frac{1}{\ln \frac{\sigma_{0}}{\sigma_{f}}}\right)}
$$


Resulting Final Equations:

$$
\begin{gathered}
u_{\text {filmA }}=U_{\max } \frac{\ln \frac{s}{S_{o}}}{\ln \frac{S_{o}}{S_{f}}+\frac{1}{2} \frac{\mu_{A}}{\mu_{B}}} \\
u_{\text {Core } B}=U_{\max }\left[\left(1-\frac{s^{2}}{S_{f}^{2}}\right)+\frac{\frac{s^{2}}{S_{f}{ }^{2}}}{\left.\left(1+\frac{1}{2} \frac{\mu_{A}}{\mu_{B}} \frac{1}{\ln \frac{S_{o}}{S_{f}}}\right)\right]}\right.
\end{gathered}
$$

Both equations are a function of $\mathrm{x}$, resulting in different profiles at different locations of $\mathrm{x}$. Each of the functionso for $\mathrm{s}$ have been described in the geometry section. The functions must be used in the correct location in the channel. or, non-dimensionalized

$$
C a_{A}=C a_{A \max }\left[\left(1-\frac{\sigma^{2}}{\sigma_{f}^{2}}\right)+\frac{\frac{\sigma^{2}}{\sigma_{f}^{2}}}{\left(1+\frac{1}{2} \frac{\mu_{A}}{\mu_{B}} \frac{1}{\ln \frac{\sigma_{o}}{\sigma_{f}}}\right)}\right]
$$

where,

$$
C a_{A}=U_{\max } \mu_{A} / \sigma_{A}
$$

C.2.2 Final Forms of Drag

Drag force is the shear stress acting over the surface area.

Shear stresses,

$$
\begin{aligned}
\tau_{w \text { Core } A} & =-2 \mu_{A} \frac{U_{\max }}{S_{o}} \\
\tau_{w \text { FilmA }} & =-\mu_{A} \frac{U_{A B}}{S_{o} \ln \left(\frac{S_{o}}{S_{f}}\right)} \\
\tau_{f_{\text {Core }}} & =-2 \mu_{B} \frac{\left(U_{\max }-U_{A B}\right)}{S_{f}}
\end{aligned}
$$

where,

$$
U_{A B}=\frac{U_{\max }}{\left(1+\frac{1}{2} M \frac{1}{\ln \left(\frac{S_{o}}{S_{f}}\right)}\right)}
$$

where, $M=\frac{\mu_{A}}{\mu_{B}}$. 
non-dimensionalized distance,

$$
\begin{aligned}
\tau_{w \text { Core } A} & =-2 \mu_{A} \frac{U_{\max }}{\sigma_{o}} \\
\tau_{w \text { FilmA }} & =-\mu_{A} \frac{U_{A B}}{\sigma_{o} \ln \left(\frac{\sigma_{o}}{\sigma_{f}}\right) R} \\
\tau_{f_{\text {Core } B}} & =-2 \mu_{B} \frac{\left(U_{\max }-U_{A B}\right)}{\sigma_{f} R}
\end{aligned}
$$

Where,

$$
U_{A B}=\frac{U_{\max }}{\left(1+\frac{1}{2} M \frac{1}{\ln \left(\frac{\sigma_{o}}{\sigma_{f}}\right)}\right)}
$$

where, $M=\frac{\mu_{A}}{\mu_{B}}$.

C.2.2.1 Core $A$

$$
\begin{aligned}
F_{\text {drag }_{\text {Core } A}} & =\iint \tau_{w \text { CoreA }} \partial x \partial z \\
& =l_{A} \int \tau_{w \text { Core } A} \partial x
\end{aligned}
$$

A closed form solution exists for the leading slug, Core A.

$$
F_{\text {drag }_{\text {coreA }}}=-16 l_{A} U_{\text {max }} \mu_{A} \ln (1+\sqrt{2})
$$

\section{C.2.2.2 Film $A$ and Core $B$}

Film A and Core B must be numerically integrated to find the values for the drag. The viscosity ratio must be included in the integration making each solution unique to the fluid pairs.

Core B:

$$
\begin{aligned}
F_{\text {drag }_{\text {Core B }}} & =\iint \tau_{f_{\text {Core B }}} \partial x \partial z \\
& =l_{b} \int \tau_{f_{\text {Core B }}} \partial x
\end{aligned}
$$

Film A:

$$
\begin{aligned}
F_{\operatorname{drag}_{\text {FilmA }}} & =\iint \tau_{w \text { FilmA }} \partial x \partial z \\
& =l_{b} \int \tau_{w \text { FilmA }} \partial x
\end{aligned}
$$


The numerical integration has to take place over different domains. The domains are the flat region and the corner. The interface that the shear stress acts over needs to be integrated over the distance normal to the surface. The distance equations can be found above C.1.2.

The non-dimensional form was used so the integration was from 0-1 for Core A and from $0-\zeta_{\text {trans }}$ for the flat region and $\zeta_{\text {trans }}-1$ for the corner region. The substitution of the distance equations and $U_{A B}$ result in equations that cannot be solved analytically but require numerical integration.

Coefficients were used for the solution of the drag forces, the drag from Core B and Film A were combined and $\alpha$ was used for the coefficient. Core A has a closed form solution but $\beta$ was used for the coefficient for consistency.

$$
\begin{aligned}
F_{\text {drag }_{\text {Core } A}} & =\beta \mu_{A} w U_{\text {max }} L_{A} \\
F_{\text {drag }_{\text {Core B }}}+F_{\text {drag }_{\text {FilmA } A}} & =\alpha U_{\text {max }} L_{B}
\end{aligned}
$$

\section{C.3 Force Balance}

Setting the motive force equal to the drag and stretching.

$$
F_{\text {motive }}=F_{\text {drag }}+F_{\text {stretch }}
$$

$$
\begin{aligned}
& \frac{2\left(\sigma_{A}-\sigma_{B}-\sigma_{A B}\right)}{R}\left(\pi R^{2}\right)= \\
& \alpha U_{\max } L_{B}+\beta U_{\max } L_{A} \mu_{A} \\
& +2 \pi R \sigma_{A}\left[\left(1-\frac{h_{A}}{R}\right)\left(\frac{\sigma_{A B}+\sigma_{B}}{\sigma_{A}}-1\right)-\frac{x_{f}}{R}\left(1-\frac{4}{\pi}\right)\left(\frac{\sigma_{A B}+\sigma_{B}}{\sigma_{A}}\right)+\frac{h_{B}}{R} \frac{\sigma_{B}}{\sigma_{A}}\right] \\
& U_{\text {max }} L_{A} \mu_{A}\left(\frac{\alpha}{\mu_{A}} \frac{L_{B}}{L_{A}}+\beta\right)= \\
& 2 \pi R \sigma_{A}\left[\left(1-\frac{\sigma_{A B}+\sigma_{B}}{\sigma_{A}}\right)\left(2-\frac{h_{A}}{R}\right)+\frac{x_{f}}{R}\left(1-\frac{4}{\pi}\right)\left(\frac{\sigma_{A B}+\sigma_{B}}{\sigma_{A}}\right)\right](\text { C. } 44)
\end{aligned}
$$

The final form has been non-dimensionalized using $C a_{A}$, assumes $h_{B} \ll 1$ to simplifiy the equation and the film thickness is found by using the Bretherton relationship.

$$
\begin{aligned}
& C a_{A \max }=\frac{U_{m} a x \mu_{A}}{\sigma_{A}}= \\
& \frac{2 \pi R}{L_{A}} \frac{\left[\left(1-\frac{\sigma_{A B}+\sigma_{B}}{\sigma_{A}}\right)\left(2-\frac{h_{A}}{R}\right)+\frac{x_{f}}{R}\left(1-\frac{4}{\pi}\right)\left(\frac{\sigma_{A B}+\sigma_{B}}{\sigma_{A}}\right)\right]}{\left(\frac{\alpha}{\mu_{A}} \frac{L_{B}}{L_{A}}+\beta\right)}
\end{aligned}
$$


Table C.1. Coefficients for $F_{d r a g}$ in the square channels

\begin{tabular}{|l|l|l|l|}
\hline Ca Number & $h_{o}(\mu \mathrm{m})$ & $\alpha(\mathrm{kg} / \mathrm{m} \mathrm{s})$ & $\beta$ \\
\hline 2 E-3 & 10.6 & 0.52567 & 14.1020 \\
4 E-3 & 16.9 & 0.51926 & 14.1020 \\
\hline
\end{tabular}

Without Stretch term:

$$
C a_{A m} a x=\frac{2 \pi R}{L_{A}} \frac{1-\left(\frac{\sigma_{A B}+\sigma_{B}}{\sigma_{A}}\right)}{\frac{\alpha}{\mu_{A}} \frac{L_{B}}{L_{A}}+\beta}
$$




\section{Appendix D}

\section{Matlab Code}

\section{D.1 Graphing}

Modeling the velocity profiles in both round and square channels with and without a thin film

Stepping Through Velocities,

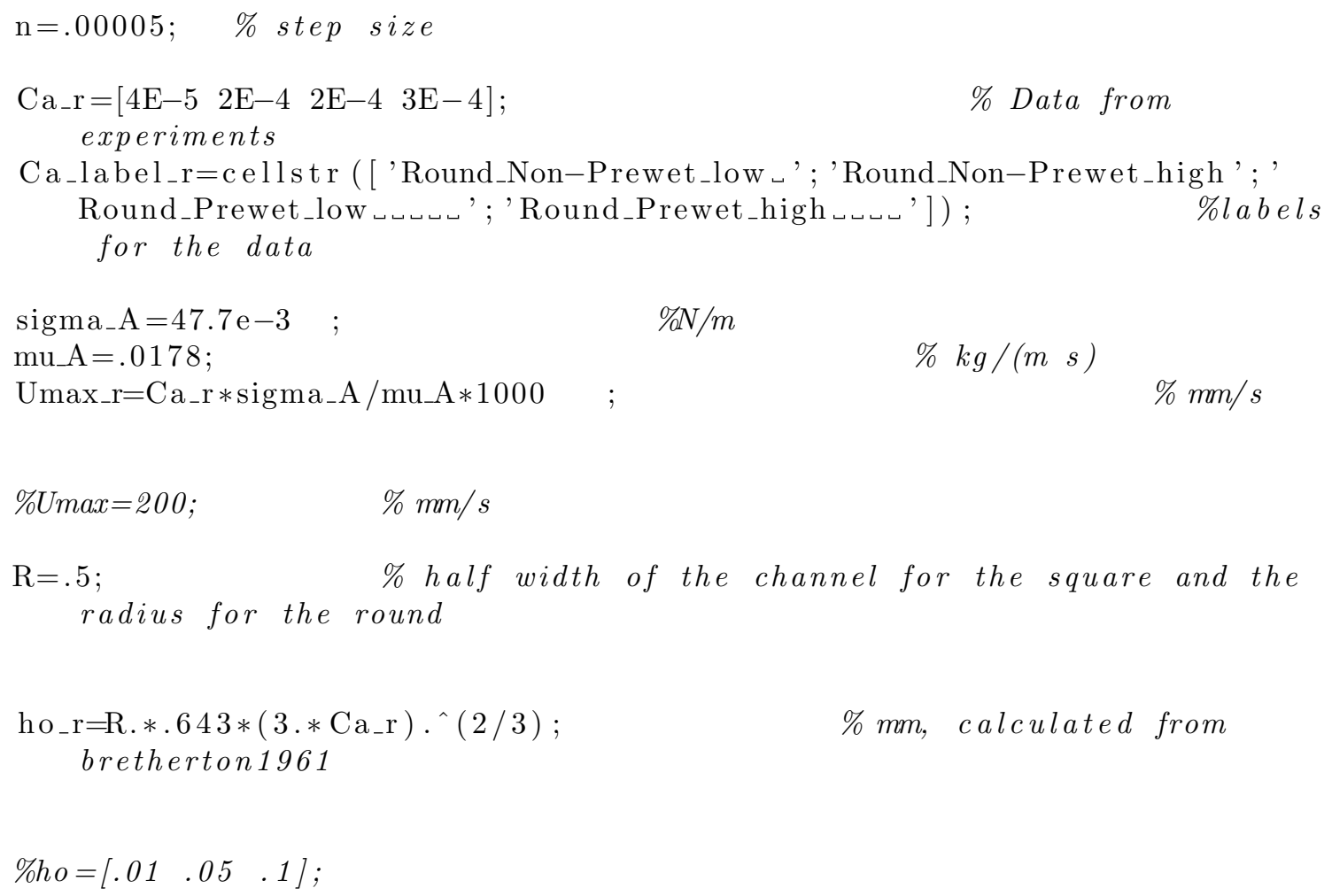

Outer Loop, Stepping Through ho

for $i=1$ :length $\left(h_{o_{-}} \mathrm{r}\right)$; 


\section{Round Channels}

Properties For a bislug system the front and film are function of fluid A and the rear is a function of fluid $B$

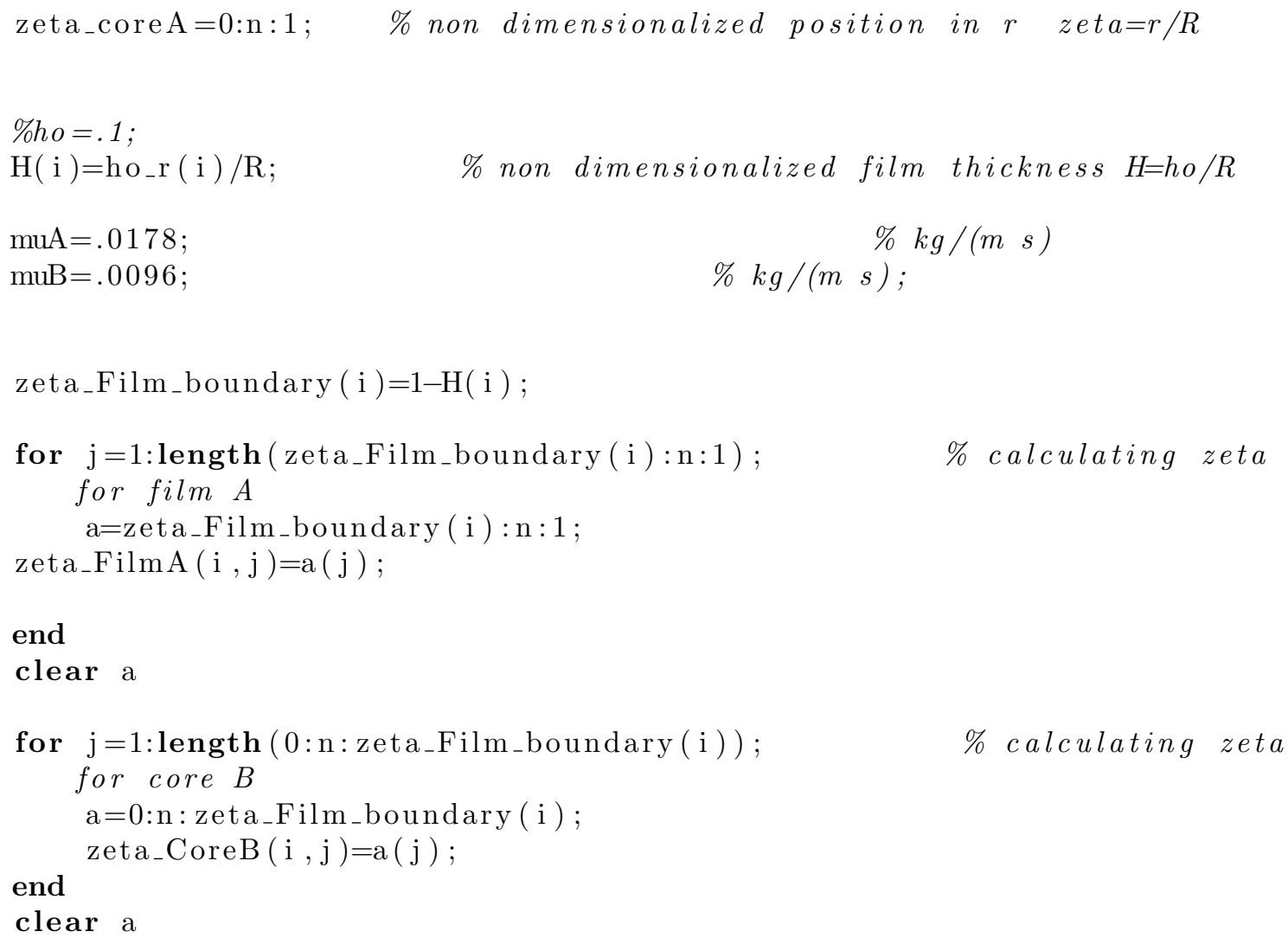

Poiseuille flow $\left(\operatorname{ur}=\operatorname{Umax}\left(1-\right.\right.$ zeta $\left.\left.^{\wedge} 2\right)\right)$

for $\mathrm{j}=1$ : length (zeta_coreA)

$\mathrm{ur}_{-} \operatorname{CoreA}(\mathrm{i}, \mathrm{j})=\operatorname{Umax} \_\mathrm{r}(\mathrm{i}) *\left(1-\right.$ zeta_coreA $\left._{(\mathrm{j})} .^{\wedge} 2\right) ; \%$ ur_A is the flow in the round channel w.r.t. fluid A end $\%$ doesn't need loop, only one series of values because it is in Core $A$

Velocity of film

$\operatorname{UABr}($ i $)=\operatorname{Umax} \_r($ i $) . /(1-1 / 2 * \operatorname{muA} / \mathrm{muB} . * 1 . / \log (1-\mathrm{H}(\mathrm{i})))$;

Poiseuille with film

for $\mathrm{j}=1$ :length (zeta_CoreB $(\mathrm{i},:))$

ur_CoreB ( i , j ) =Umax_r ( i ) * ((1+zeta_CoreB ( i , j ) ^ $2 /(1-\mathrm{H}(\mathrm{i})) \wedge 2 *(1 /(1-\mathrm{muA} /(2 *$ $\mathrm{muB}) * 1 / \log (1-\mathrm{H}(\mathrm{i})))-1)))$;

end

Couette Flow in thin film 


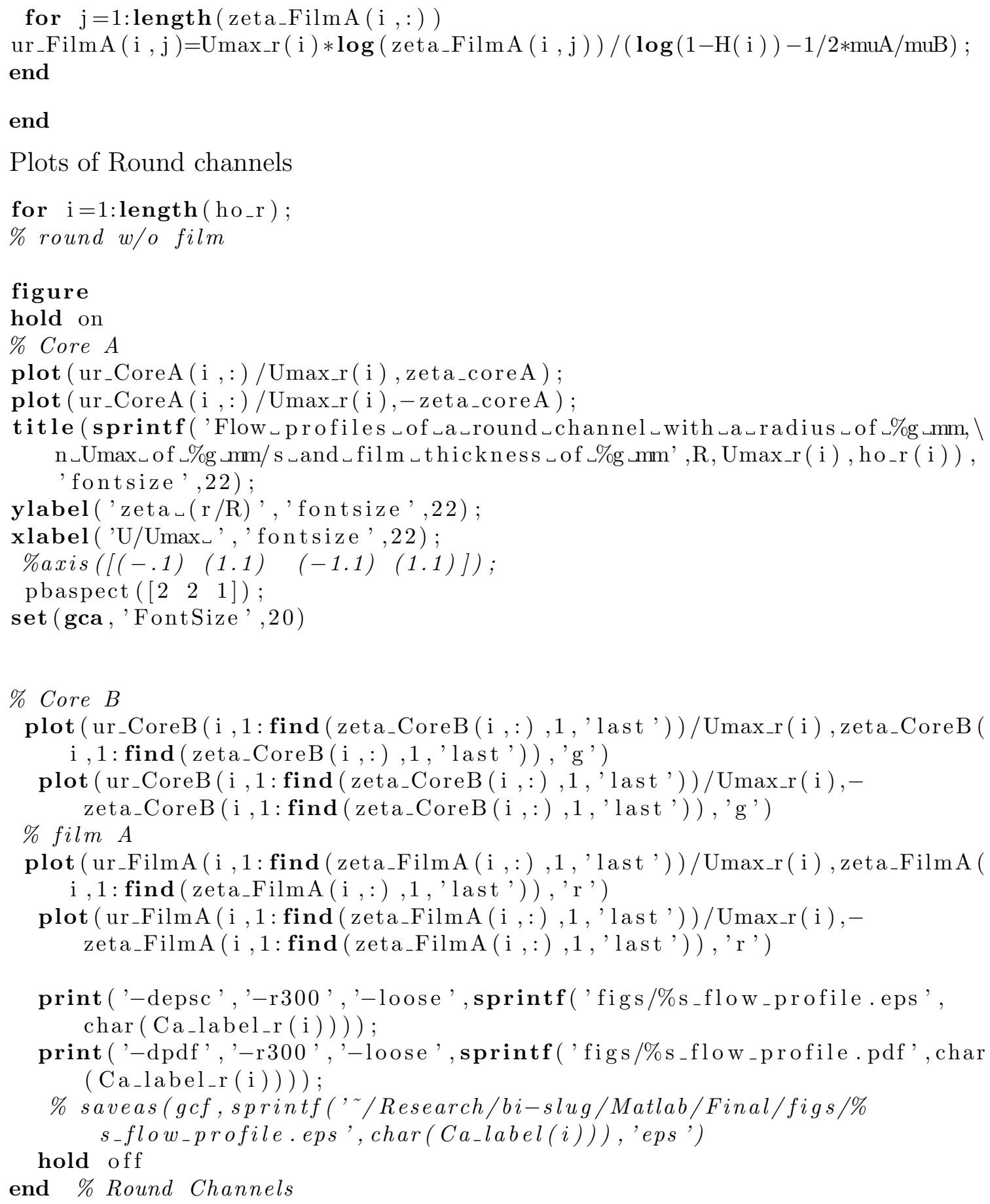

Square Channels

Going to use the $\mathrm{x}$ value from the wall as the reference point for each calculation. 
Plots of profile on $\mathrm{x}$ and the normalized film velocity will be plotted.

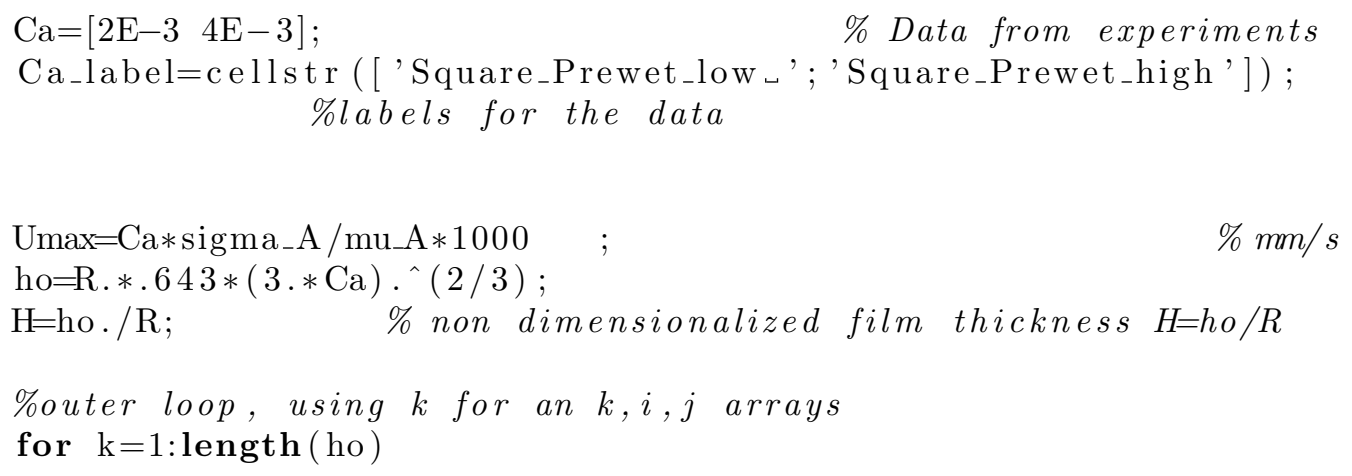

\section{Parameters}

Using the same initial parameters as the round channels

Flow in a square channel with a thin film

Two regions for both the Couette and Poiseuille flow There are two different functions for the distance to the film.

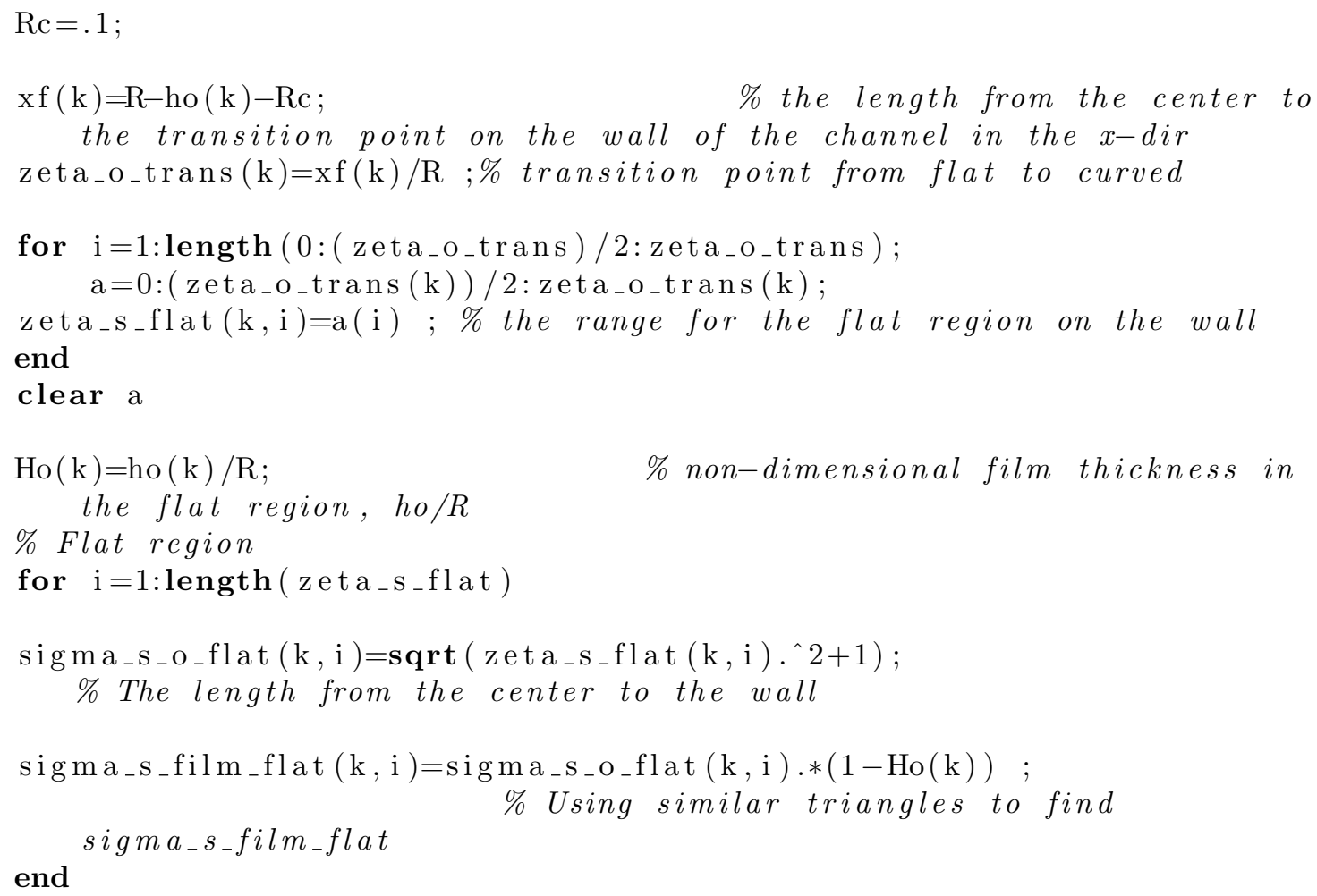




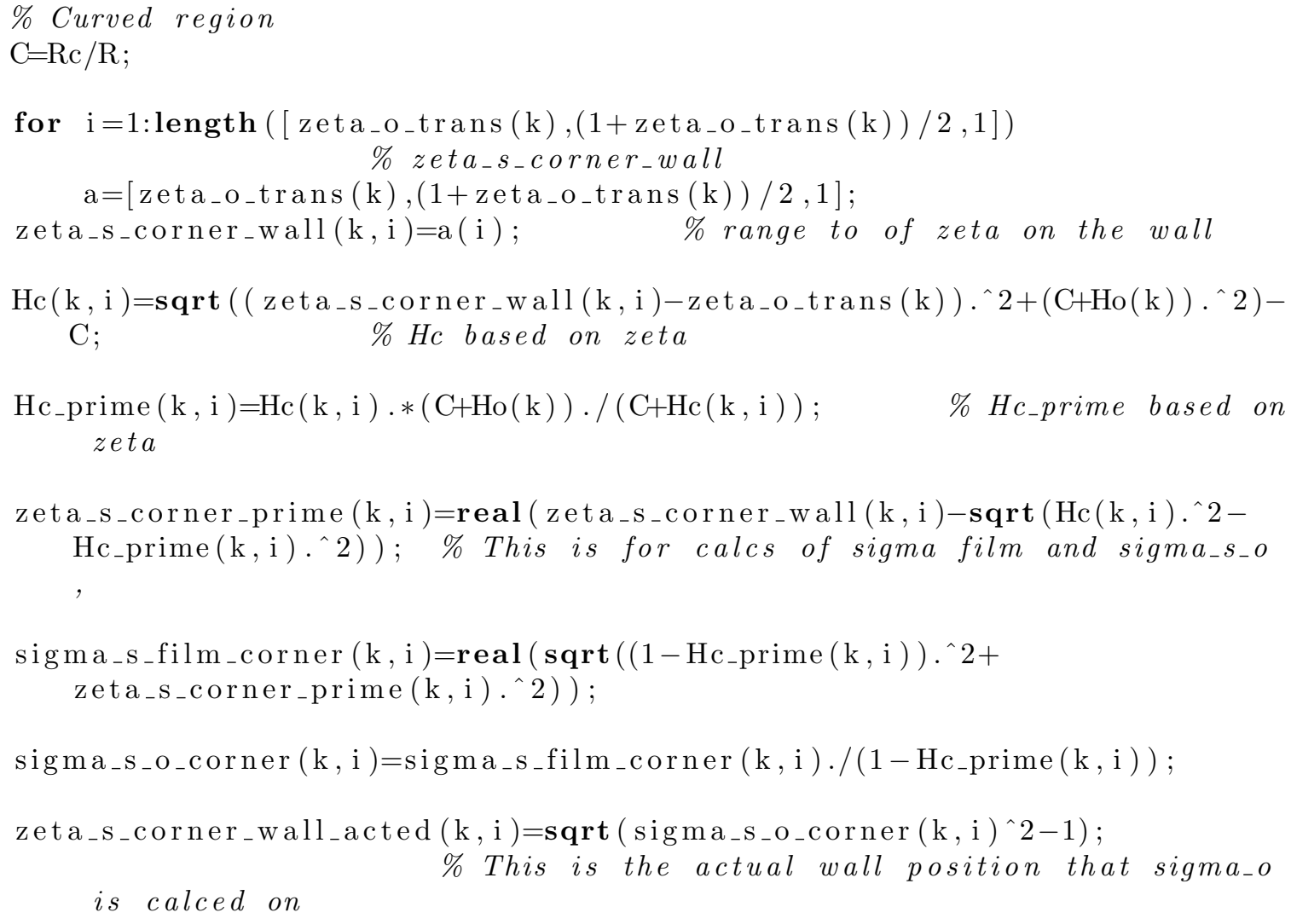

Velocity at the film, UAB for calcs

Flat

UAB_s_flat $(\mathrm{k}, \mathrm{i})=\operatorname{Umax}(\mathrm{k}) \cdot /\left(1-1 / 2 . * \mathrm{muA} / \mathrm{muB} . * 1 . / \log \left(\operatorname{sig} \mathrm{ma}_{-} \mathrm{s}_{-}\right.\right.$film_flat $(\mathrm{k}, \mathrm{i})$ ./ sigma_s_o_flat (k,i)));

\% Corner

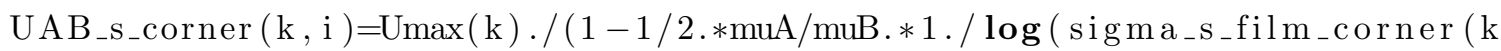
, i )./sigma_s_o_corner $(\mathrm{k}, \mathrm{i})))$;

end

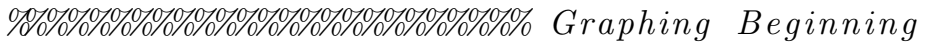

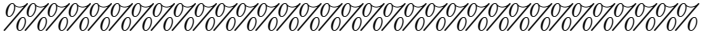

Velocity at the film, UAB for graphing

Flat 


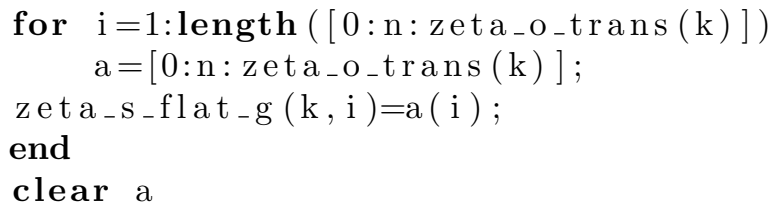

UAB_s_flat_g $(\mathrm{k}, \mathrm{i})=\operatorname{Umax}(\mathrm{k}) \cdot /(1-1 / 2 . * \mathrm{muA} / \mathrm{muB} . * 1 . / \log ($ sig m a _ s_film_flat_g $(\mathrm{k}$ , i )./ sigma_s_o_flat_g $(\mathrm{k}, \mathrm{i})))$;

end

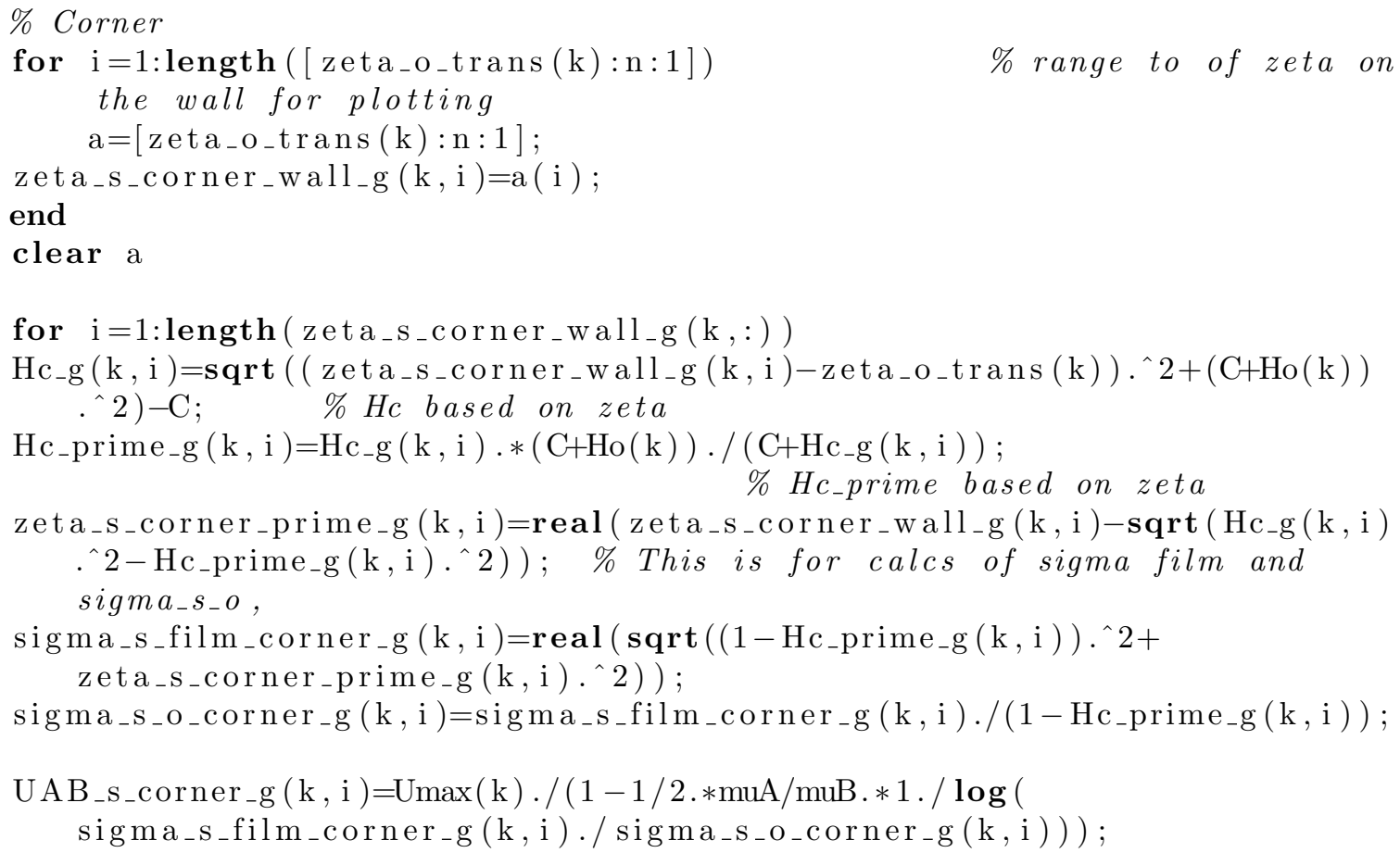

end

\%,\%\%\%\%\%\%\%\%\%\%\%\%\%\%\%\%\%\%\%\%\%\% Graphing End \%\%\%\%\%\%\%\%\%\%\%\%\%\%\%\%\%\%\%\%\%\%\%\%\%\%\%\%\%\%\% 
Poiseuille in Core B

Flat

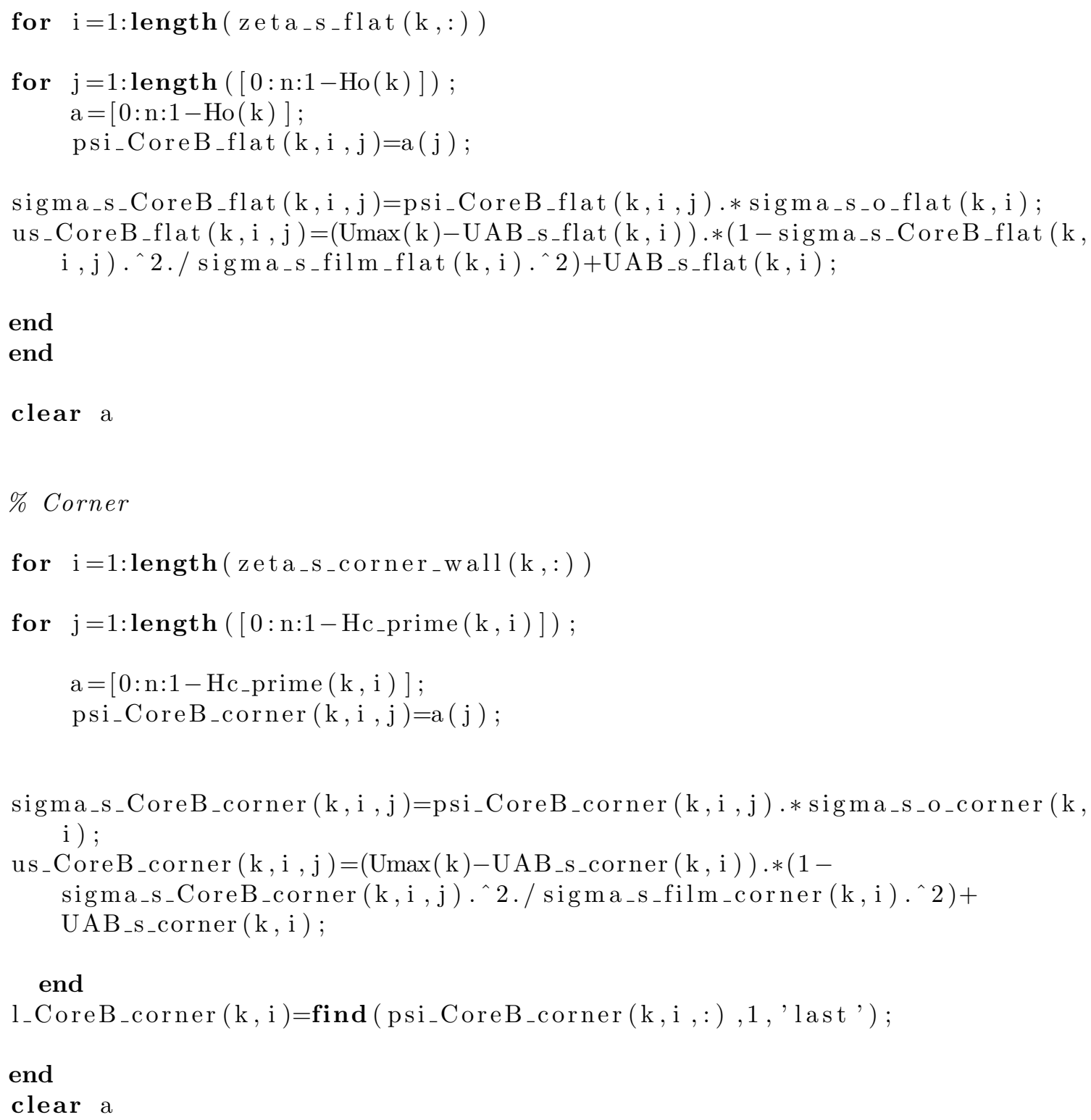

Couette

\% Flat

$$
\text { \% Boundary for the film }
$$

for $i=1$ :length $\left(\right.$ zeta $_{-} s_{-}$flat $\left.(k,:)\right)$ 


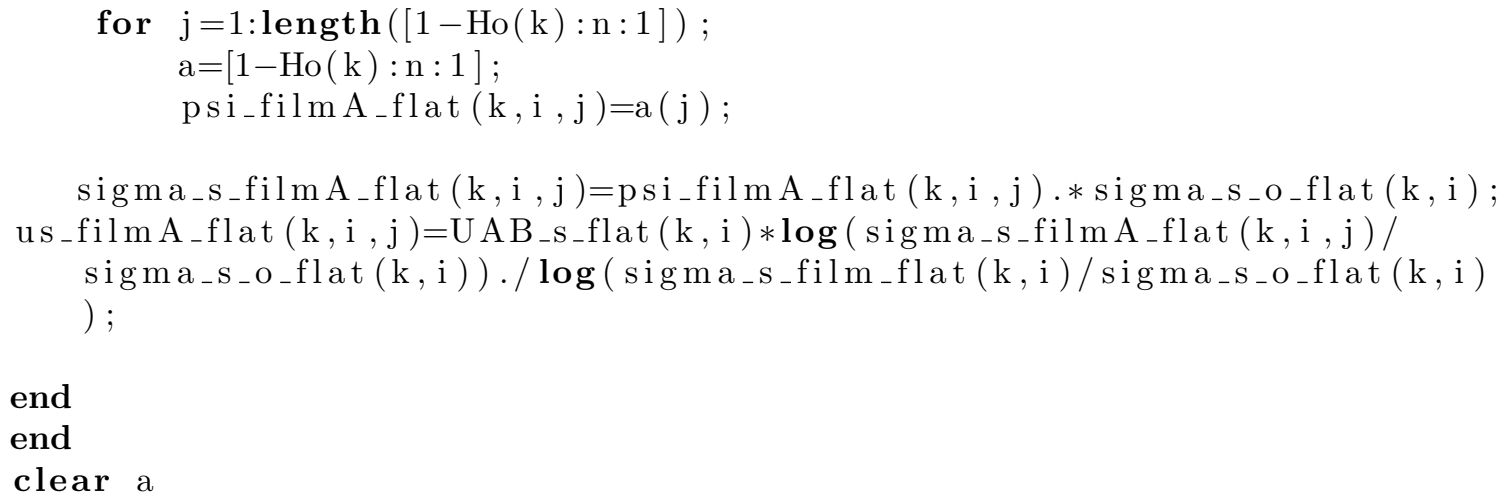




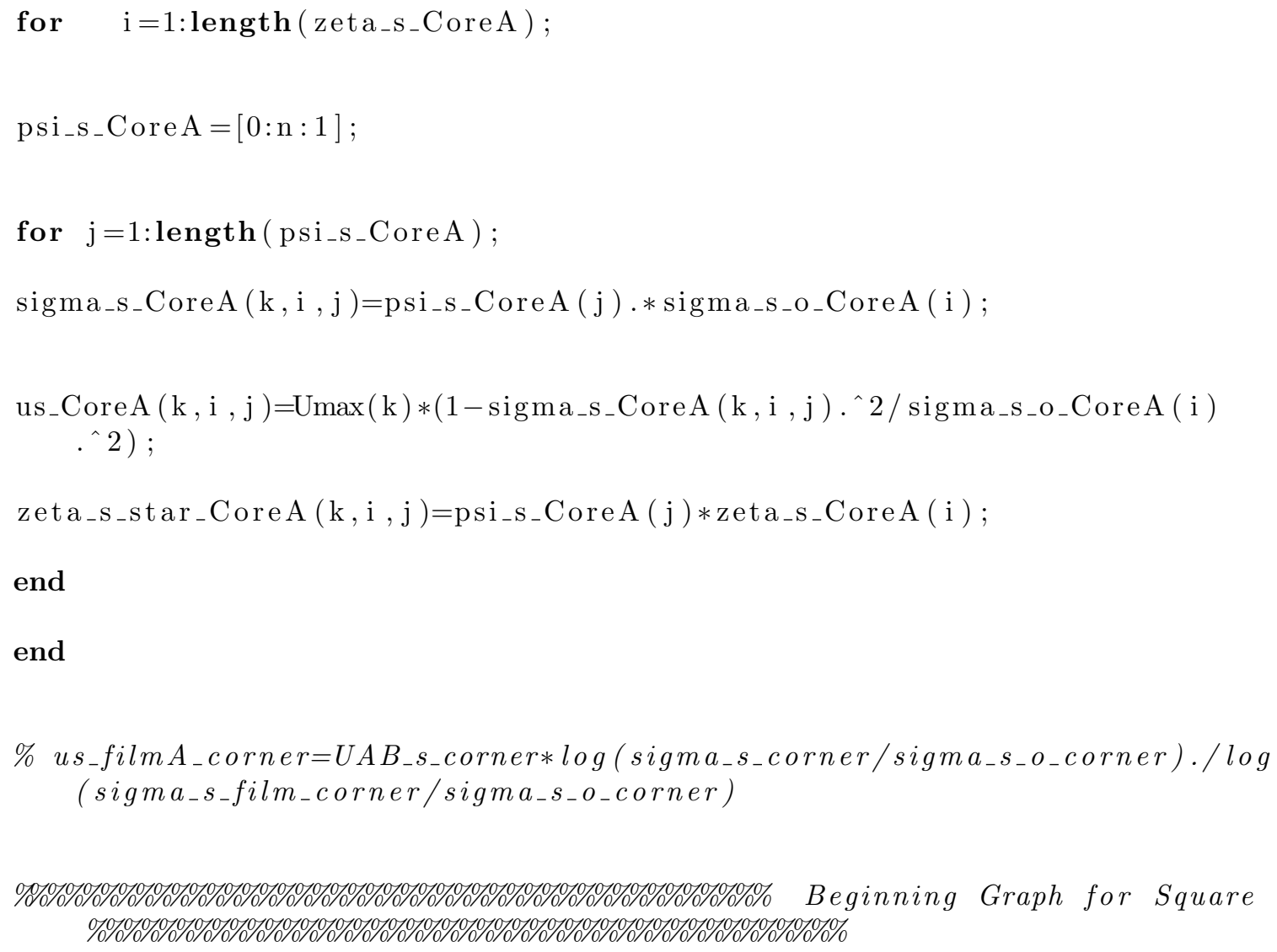

Plots in Square Channels

for $\mathrm{k}=1$ : length (ho)

Loop not needed for Core A

square $\mathrm{w} / \mathrm{o}$ film

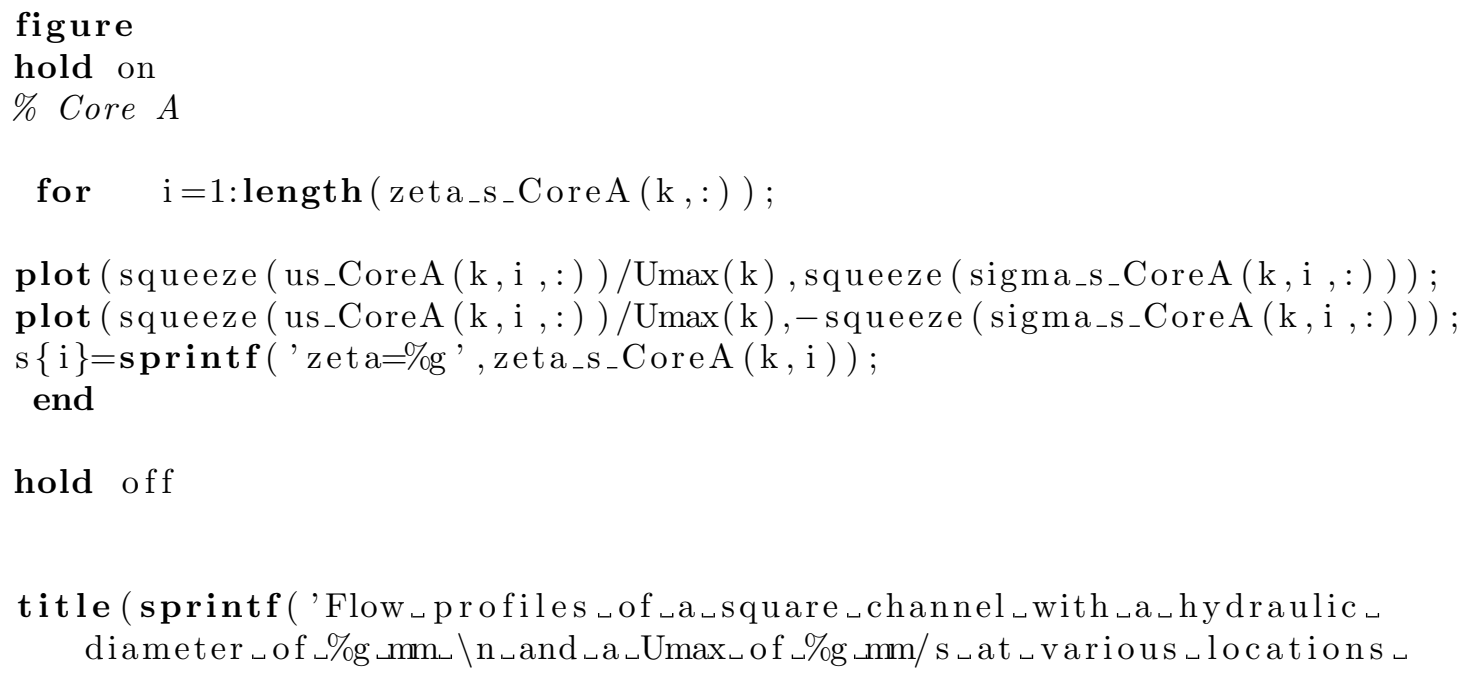




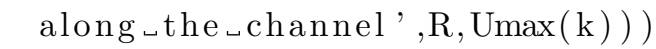

ylabel('sigma ', ' fontsize',22);

xlabel ('U/Umax ', ' fontsize', 22);

legend $(\mathrm{s})$;

legend ('boxoff', 'Best');

pbaspect $\left(\left[\begin{array}{lll}3 & 3 & 1\end{array}\right]\right)$;

print ('-depsc', '-r300', '-loose', sprintf('figs/\% s_flow_profile.eps', char( Ca_label(k))));

print ('-dpdf', '-r300', '-loose', sprintf(' figs/\% s flow_profile .pdf', char( Ca_label(k))));

end

Plots of flow in square channels with a thin film

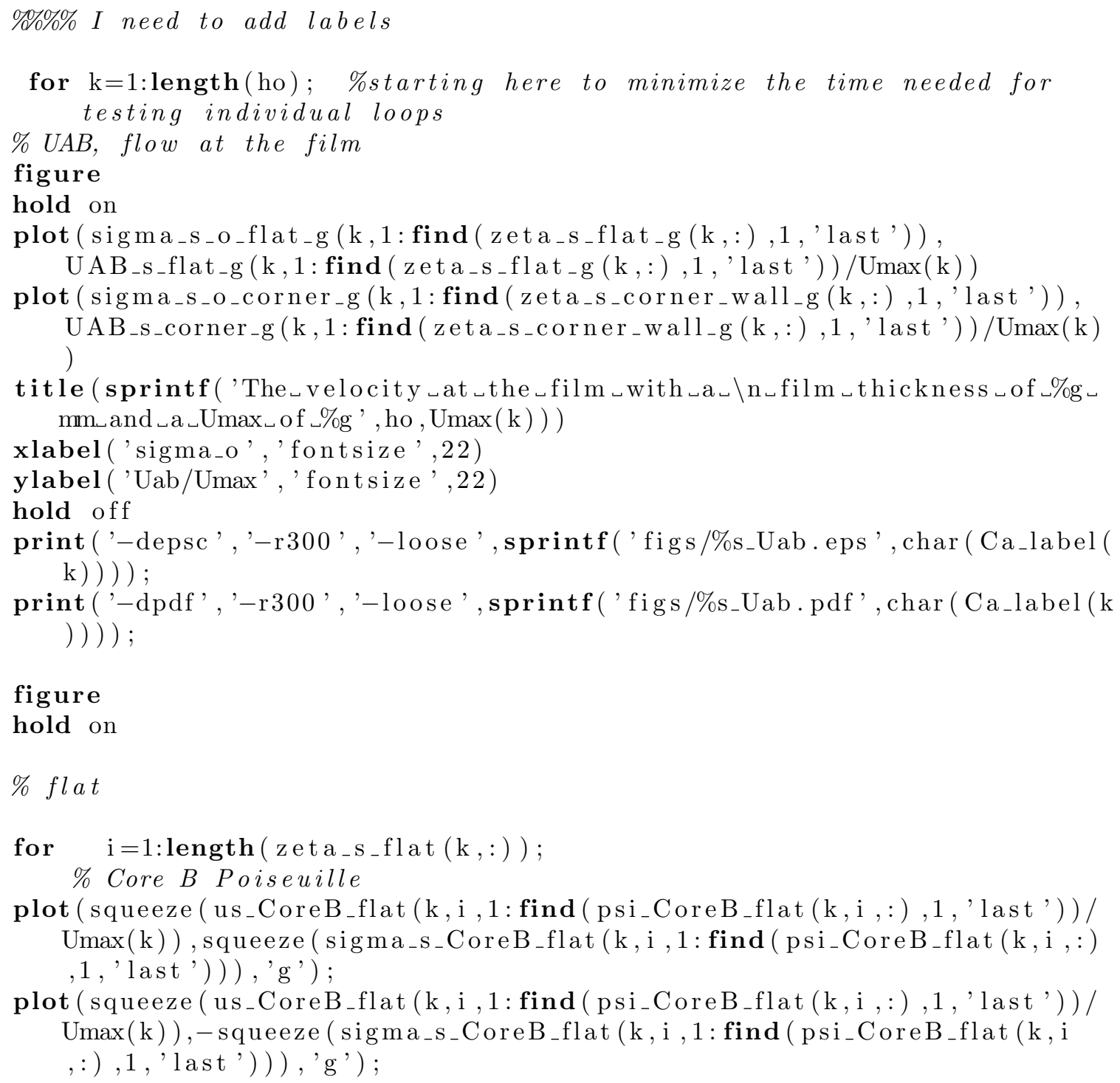


$\%$ Film A Couette flow

plot (squeeze( us_filmA_flat (k,i , 1: find (psi_filmA_flat (k, i,: ), 1, 'last')) /

$\operatorname{Umax}(\mathrm{k}))$, squeeze ( sigma_s_film $A_{-}$flat (k, i, 1: find ( psi_film $A_{-}$flat $(\mathrm{k}, \mathrm{i},:)$ ,1, 'last'))), 'r' ) ;

plot (squeeze ( us_filmA_flat (k, i, 1: find (psi_filmA_flat (k, i,: ), 1, 'last')) /

$\operatorname{Umax}(\mathrm{k})),-$ squeeze ( sigma_s_film A_flat (k,i , 1:find ( psi_film A_flat (k, i

, : ) , 1, 'last')) ), 'r' ) ;

$\mathrm{s}\{\mathrm{i}\}=$ sprintf(' zeta=\%g', zeta $\mathrm{s}_{-}$flat $\left.(\mathrm{k}, \mathrm{i})\right)$;

end

$\%$ curved

for $\quad \mathrm{i}=1$ : length $\left(\right.$ zeta $_{-} \mathrm{s}_{-} \operatorname{corner}_{-}$wall $\left.(\mathrm{k},:)\right)$;

\% Core B Poiseuille

plot ( squeeze ( us_CoreB_corner (k, i , 1: l_CoreB_corner (k, i )) /Umax (k)), squeeze ( $\left.\operatorname{sigma} \mathrm{s}_{-} \mathrm{CoreB}_{-} \operatorname{corner}\left(\mathrm{k}, \mathrm{i}, 1: \mathrm{l}_{-} \mathrm{CoreB}_{-} \operatorname{corner}(\mathrm{k}, \mathrm{i})\right)\right)$, 'b') ;

plot (squeeze ( us_CoreB_corner (k, i, 1: l_CoreB_corner (k, i )) /Umax $(k))$, -

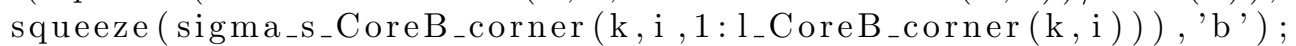

$\%$ Film A Couette flow

plot (squeeze( us_film A_corner (k, i, 1: l_filmA_corner (k, i )) /Umax(k)), squeeze ( sigma_s_film A_corner (k,i,1: l_filmA_corner (k, i ))), 'r') ;

plot (squeeze(us_filmA_corner (k,i,1: l_filmA_corner (k,i )) /Umax(k)), squeeze ( sigma_s_filmA_corner (k,i , 1: l_filmA_corner (k, i )) ), 'r ') ; $\% s\{i\}=\operatorname{sprintf}\left(' z e t a=\% g\right.$ ', zet $\left.a_{-} s_{-} f l a t(i)\right) ; \%$ need to work on this

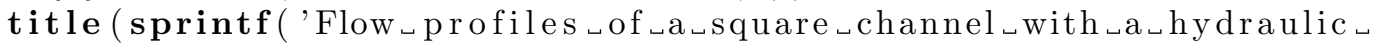

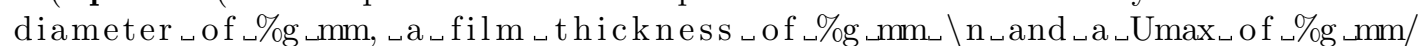

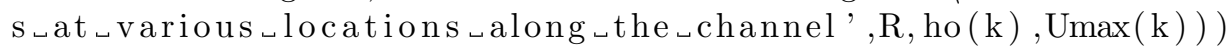

end

\% title(sprintf('Flow profiles of a square channel with a hydraulic diameter of $\% \mathrm{gmm}$, a film thickness of $\% \mathrm{gm} \backslash n$ and a Umax of \%g mm/ $s$ at various locations along the channel', $R$, ho,Umax))

ylabel ('sigma', ' fontsize',22);

xlabel ('U/Umax', ' fontsize', 22 );

legend $(\mathrm{s}) ; \% i$ need to work on the the legend

legend ('boxoff', 'Best') ;

pbaspect $\left(\left[\begin{array}{lll}3 & 3 & 1\end{array}\right]\right)$;

hold off

print ('-depsc', '-r300', '-loose', sprintf('figs/\%s_flow_profile_film .eps', $\left.\left.\operatorname{char}\left(\mathrm{Ca}_{-} \mathrm{label}(\mathrm{k})\right)\right)\right)$;

print ('-dpdf', '-r300', '-loose', sprintf('figs/\%s_flow_profile_film .pdf', $\left.\left.\operatorname{char}\left(\mathrm{Ca}_{-} \mathrm{label}(\mathrm{k})\right)\right)\right)$;

Channel Profile 


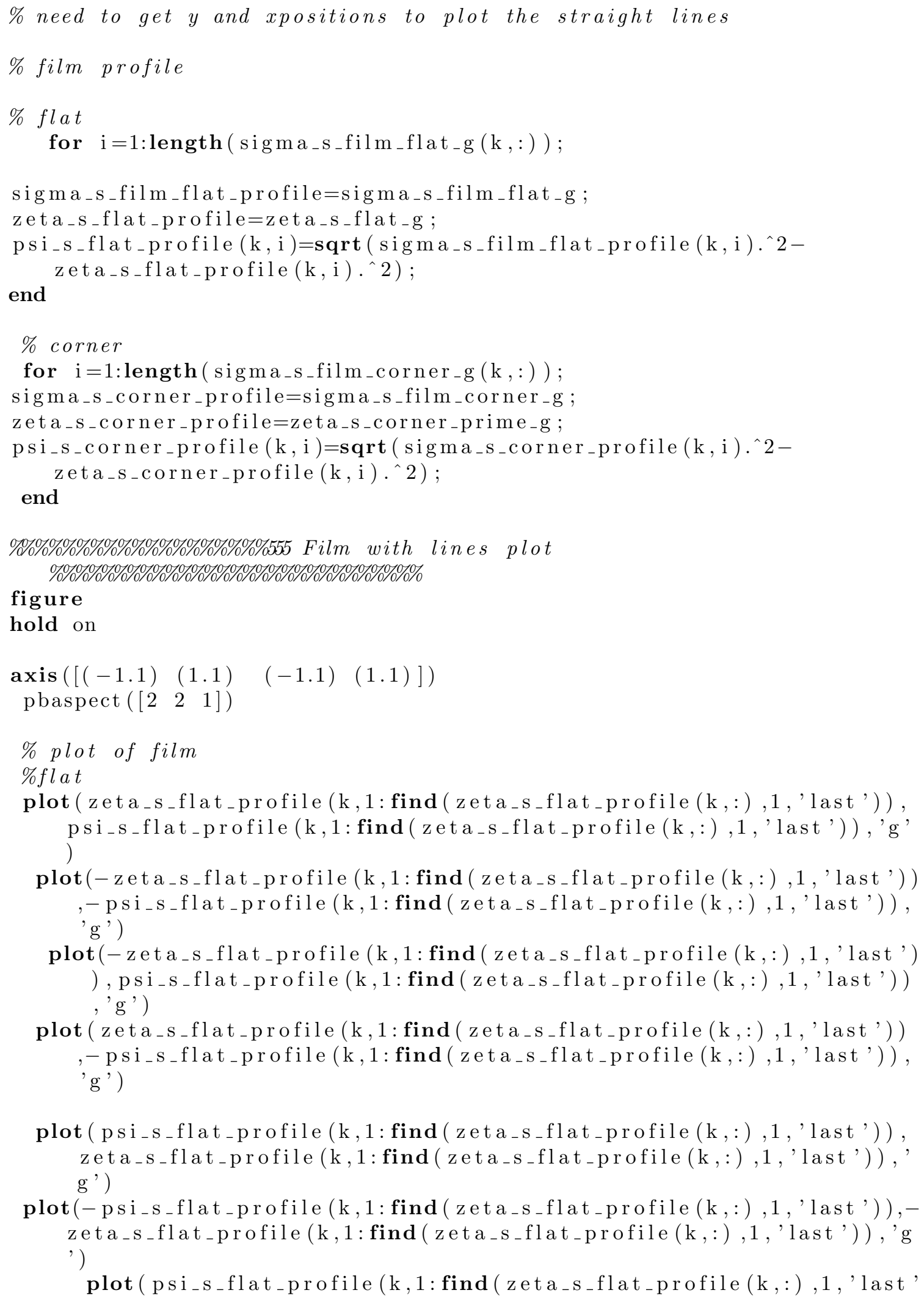




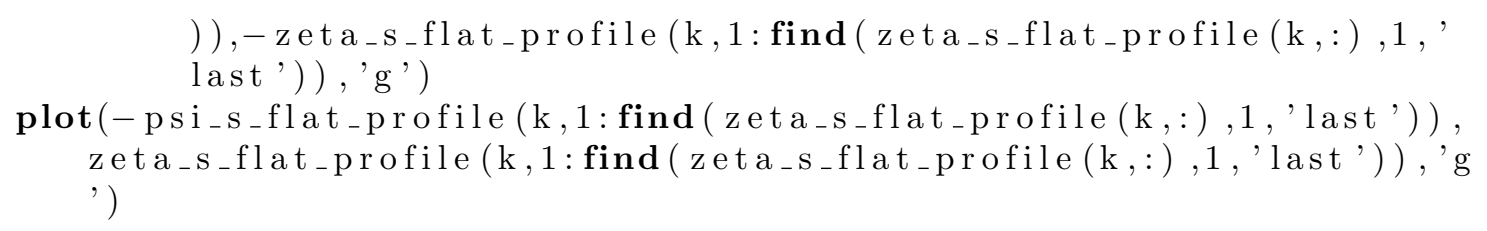




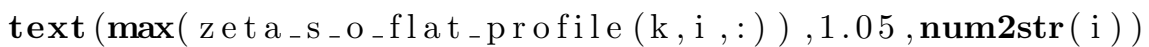

$\operatorname{leg}\{\mathrm{i}\}=\operatorname{sprintf}\left({ }^{\prime} \% \mathrm{~g}=\% \mathrm{~g},, \mathrm{i}, \max \left(\mathrm{zeta}_{-} \mathrm{s}_{-} \mathrm{o}_{-}\right.\right.$flat $\mathrm{g}_{-}$rofile $\left.\left.(\mathrm{k}, \mathrm{i},:)\right)\right)$

end

$\%$ corner

for $\quad \mathrm{i}=1$ : length $\left(\right.$ zeta $_{-} \mathrm{S}_{-}$corner_wall $\left.(\mathrm{k},:)\right)$;

psi_profile $=[0: n: 1]$;

for $\mathrm{j}=1$ :length ( $\mathrm{psi}$ profile)

zeta_s_o_corner_profile $(\mathrm{k}, \mathrm{i}, \mathrm{j})=\mathrm{psi}_{-}$profile $(\mathrm{j})$. zeta_ $_{-} \mathrm{s}_{-} \mathrm{corner}_{-} \mathrm{wall}(\mathrm{k}, \mathrm{i})$; end

plot (squeeze( zeta_s_o_corner_profile (k,i , : )), psi_profile , 'r' );

\%text (max(zeta $a_{-} s_{-} o_{-}$corner-profile $\left.(k, i,:)\right), 1,[' \backslash z$ eta $=$ ', num2str( zeta_s_corner-wall_acted $(k, i))])$;

text $\left(\max \left(\right.\right.$ zeta $_{-} \mathrm{S}_{-} \mathrm{O}_{-} \mathrm{corner}$ orofile $\left.(\mathrm{k}, \mathrm{i},:)\right), 1.05, \operatorname{num} 2 \mathbf{s t r}\left(\mathbf{l e n g t h}\left(\mathrm{zeta}_{-} \mathrm{s}_{-} \mathrm{flat}\right.\right.$ $(\mathrm{k},:))+\mathrm{i}))$;

$\operatorname{leg}\left\{\right.$ length $\left.\left(\mathrm{zeta}_{-} \mathrm{s}_{-} \mathrm{flat}(\mathrm{k},:)\right)+\mathrm{i}\right\}=\operatorname{sprintf}\left({ }^{\prime} \% \mathrm{~g}=\% \mathrm{~g}\right.$, , length $\left(\mathrm{zet}_{-} \mathrm{s}_{-} \mathrm{flat}(\mathrm{k},:)\right)$

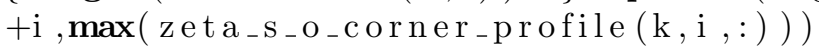

end

$\%$ boundary of box

$\mathrm{xw}$ all $=-1: \mathrm{n}: 1$

y w all $=-1: \mathrm{n}: 1$;

plot $(-1$, ywall , 'b' ,1, ywall, 'b' , xwall ,1, 'b' , xwall ,-1, 'b')

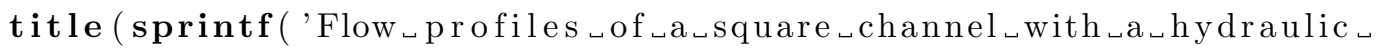

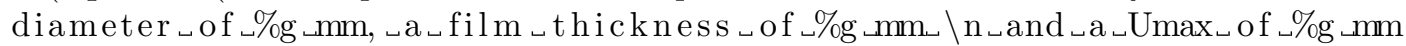

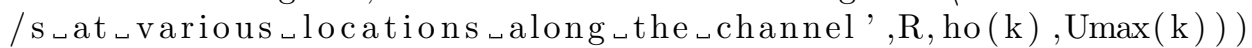

T=legend (leg, 'Location', 'SouthWest')

set (get (T, 'title'), 'String', 'Wall Intercept')

pbaspect $\left(\left[\begin{array}{lll}3 & 3 & 1\end{array}\right]\right)$;

hold off

print ('-depsc', '-r300', '-loose', sprintf(' figs/\% $/$ s_flow $_{-}$profile_lines.eps' , char $\left.\left.\left(\mathrm{Ca}_{-} \mathrm{label}(\mathrm{k})\right)\right)\right)$;

print ('-dpdf', '-r300', '-loose', sprintf(' figs/\% s_flow_profile_lines.pdf', $\left.\left.\operatorname{char}\left(\mathrm{Ca}_{-} \mathrm{label}(\mathrm{k})\right)\right)\right)$;

end

Trying to find a relationship for $\mathrm{Hc}$

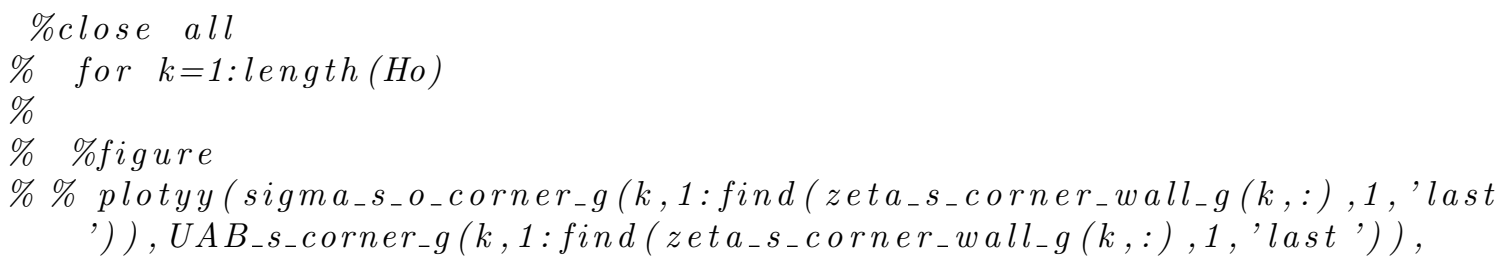




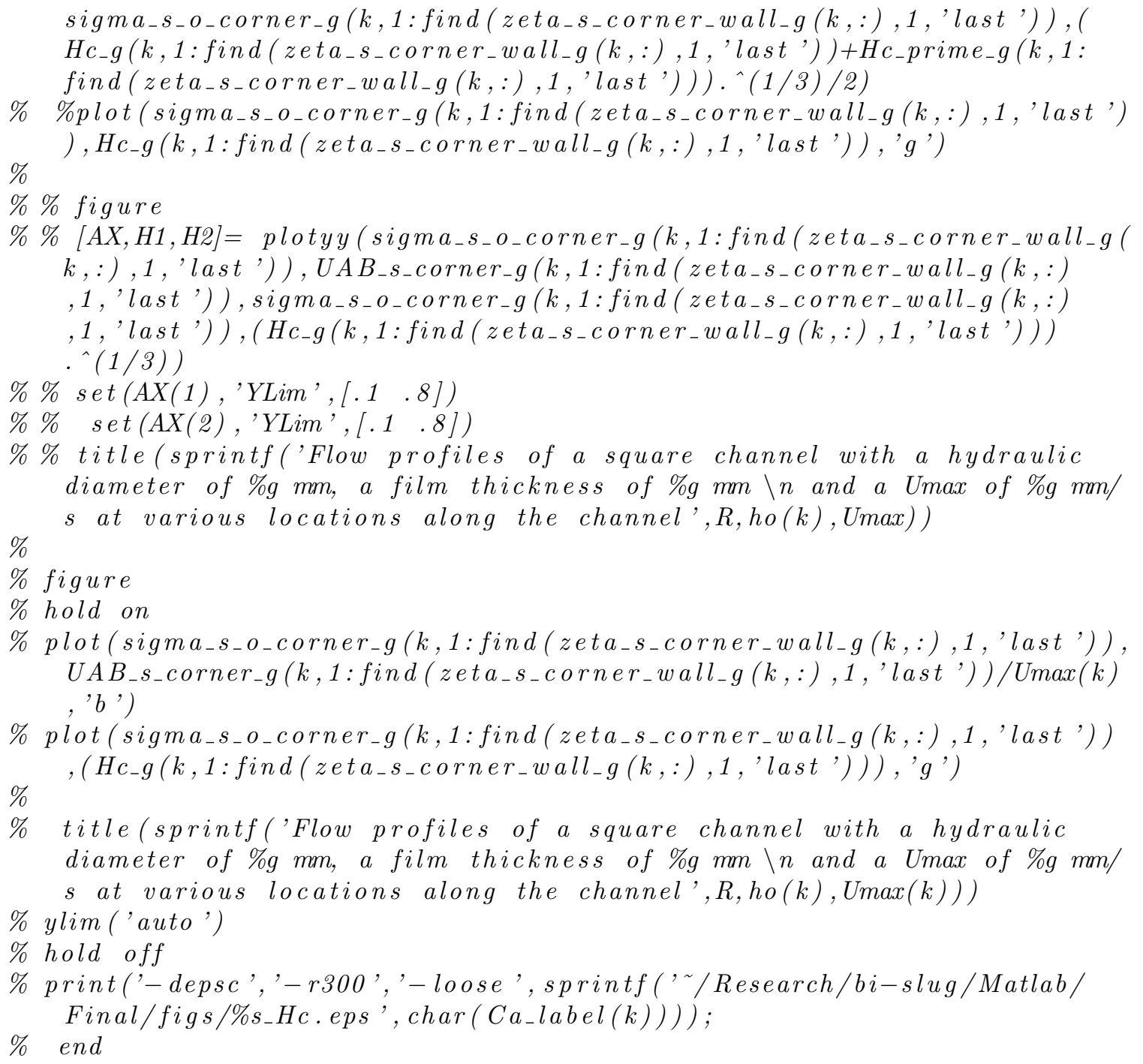

\section{D.2 Derivation}

clear, clc, close all

set ( 0 , 'DefaultFigureWindowStyle ', 'docked ')

set $(0$, 'DefaultFigurePaperOrient', 'landscape')

Integration of the different sections of the square channel for use in a model

\% Each part needs to be integrated, there may not be a closed solution $\%$ which would require numerical integration.

Flow in Core $\mathrm{A}$ in the square channel

\section{Core A Parameters}

syms u_a U_max s_a so_a mu_a zeta_a z l_a

$\% u_{-} a$ is the velocity in the channel 
\% U_max is the center line velocity

$\% s_{-} a$ is the length from the center point to any location in the channel

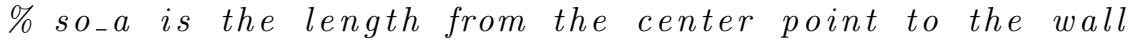

$\%$ mu_a is the viscosity of fluid $A$

$\%$ zeta_a is the non-dimensional distance on the wall, $x / R$

$\% z$ is the direction of the slug length

$\% l_{-} a$ is the length of the slug

Equations for Core A

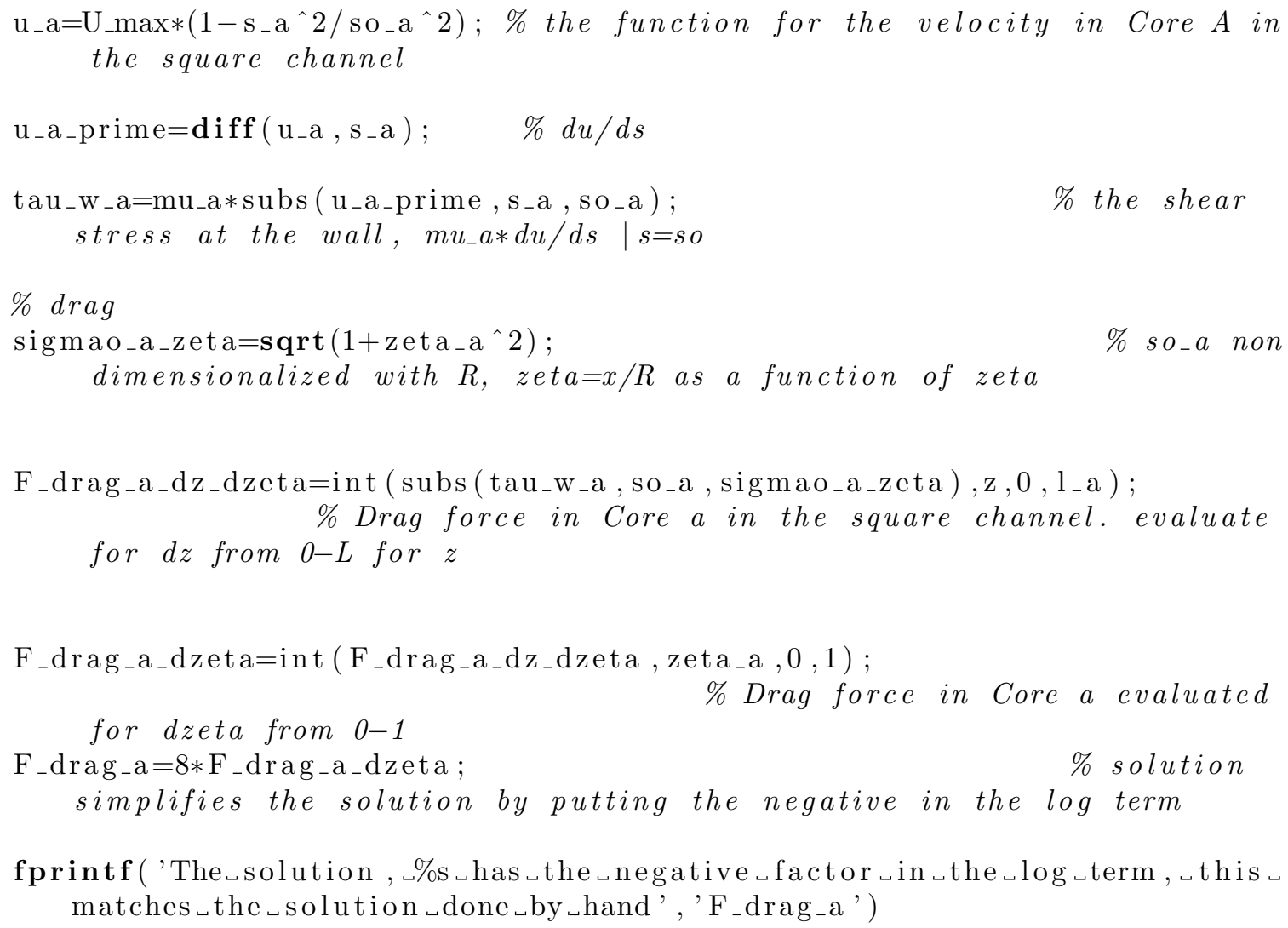

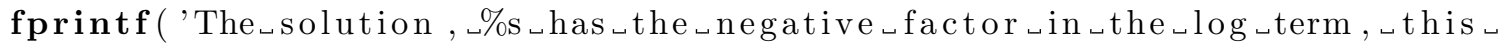

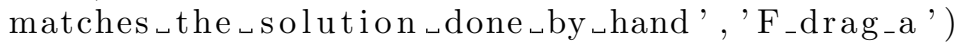

Core B and Film A

Core B and Film A are dependent on each other

\section{Core B and Film A Parameters}

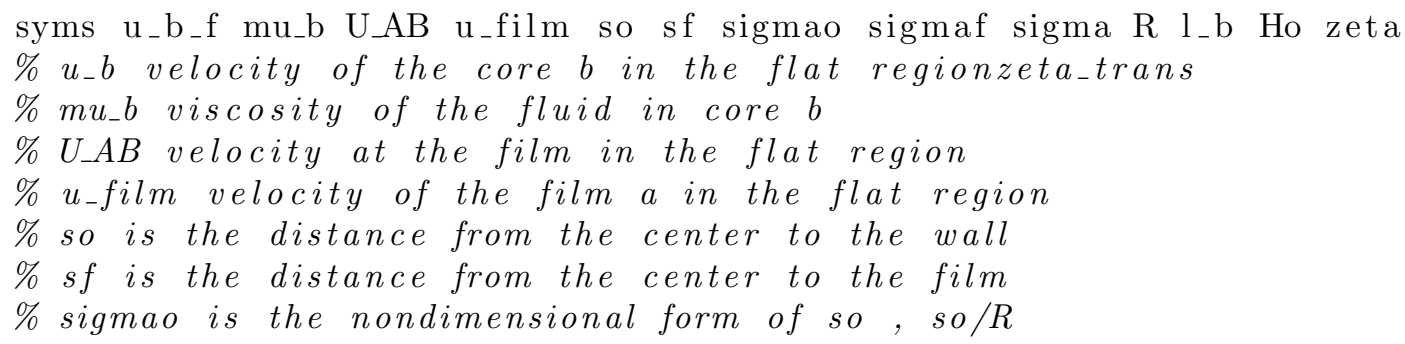


\% sigmaf is the nondimensional form of $s f, s f / R$

$\% R$ is the Length of the half the wall

$\% l_{-} b$ is the length of the slug in COre B, the film is located in this region

\% Ho nondimensionalized film thickness ho, ho/R

$\%$ zeta is the nondimensionalized distance on the wall, $x / R$

$\%$ zeta_trans is the nondimensionalized distance to the transition between the flat and curved region

Equations for the velocities

$\mathrm{U} \_\mathrm{AB}=\mathrm{U} \_\max /(1+1 / 2 * \mathrm{mu} \mathrm{a} / \mathrm{mu} \mathrm{b} * 1 / \log (\operatorname{sigmao} / \operatorname{sigmaf})) ; \quad \%$ velocity at the film in the flat region, using the non-dimensional form

$\mathrm{u}_{-} \mathrm{film}=\mathrm{U} \_\mathrm{AB} * \log (\operatorname{sigmao} / \operatorname{sigma}) / \log (\operatorname{sigmao} / \operatorname{sigmaf}) ; \quad \%$ velocity of the film as a function of sigma $f$

$\mathrm{u}_{-} \mathrm{b}=\left(\mathrm{U} \_\mathrm{max}-\mathrm{U} \_\mathrm{AB}\right) *\left(1-\operatorname{sigma}^{\wedge} 2 / \operatorname{sigmaf}^{\wedge} 2\right)+\mathrm{U} \_\mathrm{AB}$;

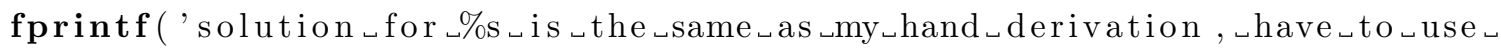

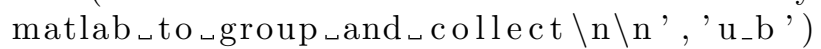

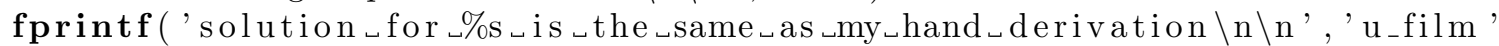
)

$\%$ find shear stress

$\mathrm{u}_{-} \mathrm{b}_{-}$prime $=\mathbf{d i f f}\left(\mathrm{u}_{-} \mathrm{b}, \operatorname{sigma}\right) / \mathrm{R} ; \quad \%$ du $/$ dsigma dsigma=ds $/ R$

$d \operatorname{sigma} * R=d s$

$\mathrm{u}_{-}$film_prime=diff $\left(\mathrm{u}_{-} \mathrm{film}, \operatorname{sigma}\right) / \mathrm{R} ; \quad \% d u / d \operatorname{sigma} \quad \operatorname{dsigma}=d s / R$

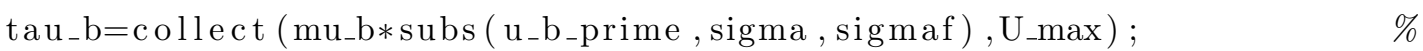

shear stress at the film, using collect on U_max to match my solutions

ta u_film=collect (mu_a*subs ( u_film_prime, sigma, sigmao),U_max) ;

$\%$ shear stress at the wall, using collect on U_max to match my solutions

\% integrating to find shear stress

\% fdrag=8*int*int tau_a dz dzeta

$\% d z$ is the differential length of the slug

$\%$ dzet $a=d x / R$

Fdrag_b_dz_dzeta=int $\left(\right.$ tau_b, z, $\left.0, l_{-} b\right)$;

Fdrag_film_dz_dzeta=int (tau_film, z,0, $\left.\mathrm{l}_{-} \mathrm{b}\right) ; \quad \%$ First integration of the drag in the flat regions, int $d z \mid 0-l_{-} b$

$\operatorname{sigmao_{-}} \mathrm{f}=\mathbf{s q r t}\left(\mathrm{zeta}^{\wedge} 2+1\right)$;

to the wall for the flat region

$\%$ distance from the center 


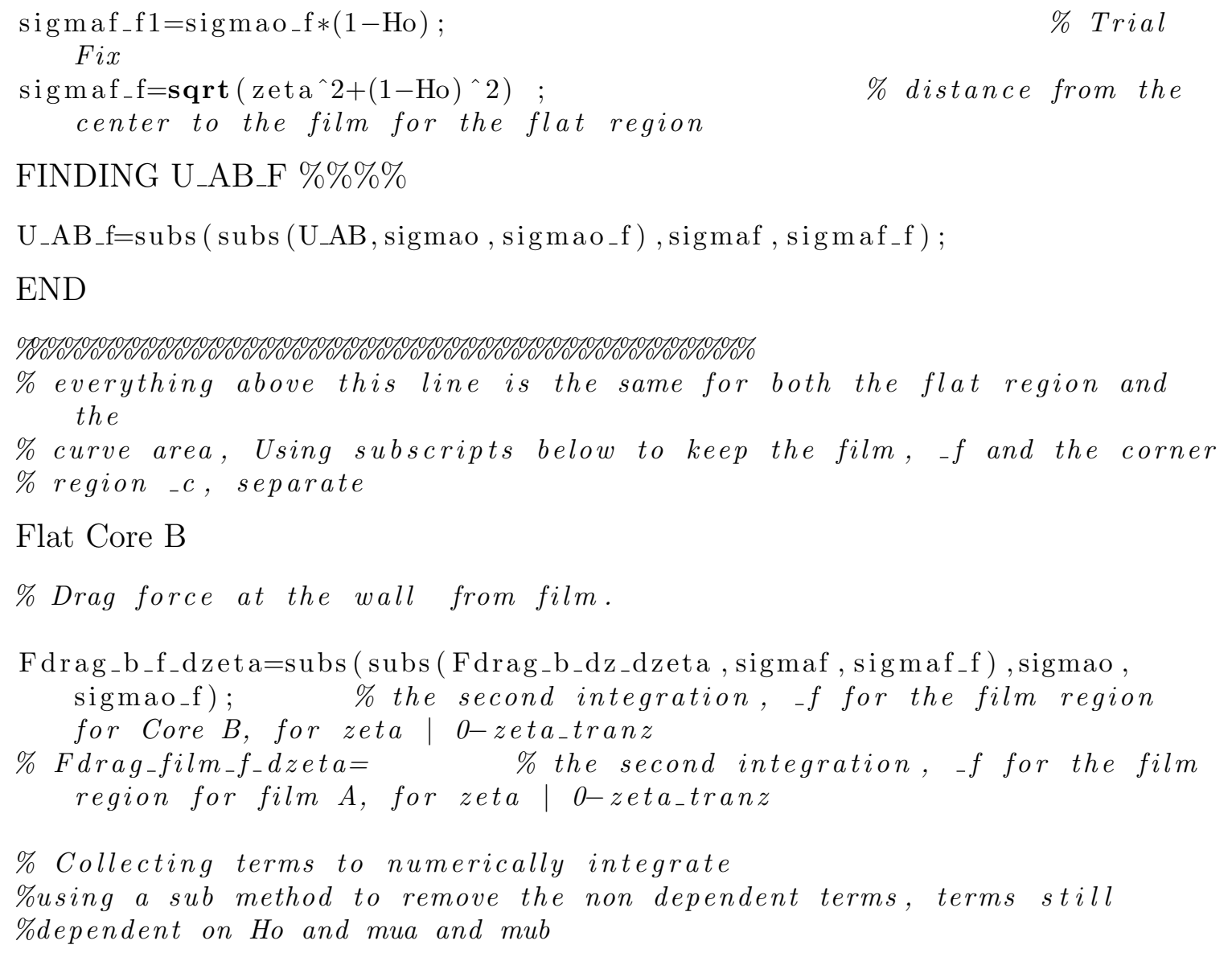




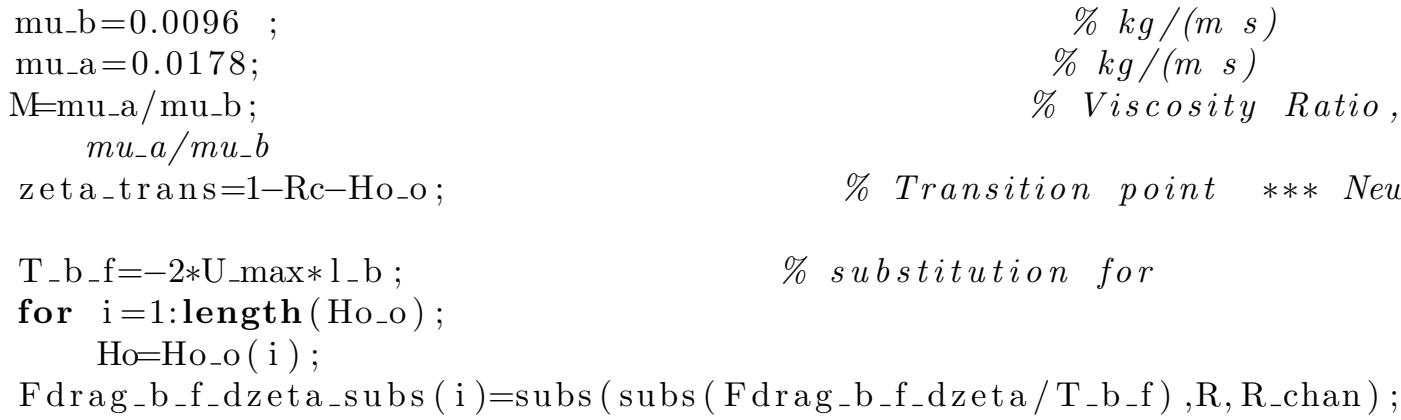

Fdrag_b_f_string=char (vectorize (Fdrag_b_f_dzeta_subs (i))); \% Convert syms to string for numerical integration

zeta_end_f ( i ) =zeta_trans (i );

Fdrag_b_f_int (i )=quad (Fdrag_b_f_string, 0 , zeta_end_f ( i ) ) ;

end

Convert back to equation form

Fdrag_b_f $=8 *\left(T_{-} b_{-} f * F d r a g b_{-} f_{-} f_{-} i n t\right) ;$

\section{Flat Film A}

Fdrag_film_f_dzeta=subs (subs (Fdrag_film_dz_dzeta, sigmaf, sigmaf_f), sigmao , sigmao_f);

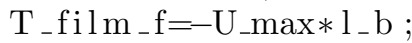

for $i=1$ :length (Ho_o $)$

$\mathrm{Ho}=\mathrm{Ho}_{-} \mathrm{O}(\mathrm{i})$;

Fdrag_film_f_dzeta_subs (i) =subs( subs(Fdrag_film_f_dzeta/T_film_f),R, $R_{-}$chan );

Fdrag_film_f_string=char (vectorize (Fdrag_film_f_dzeta_subs (i )) );

Fdrag_film_f_int (i)=quad (Fdrag_film_f_string, 0, zeta_trans (i));

end

Fdrag_film_f $=8 * T_{-} f i l m_{-} f * F d r a g f_{-} f_{l} m_{-} f_{-} i n t ;$

\%o\%

Finding U_AB_C \%\%\%

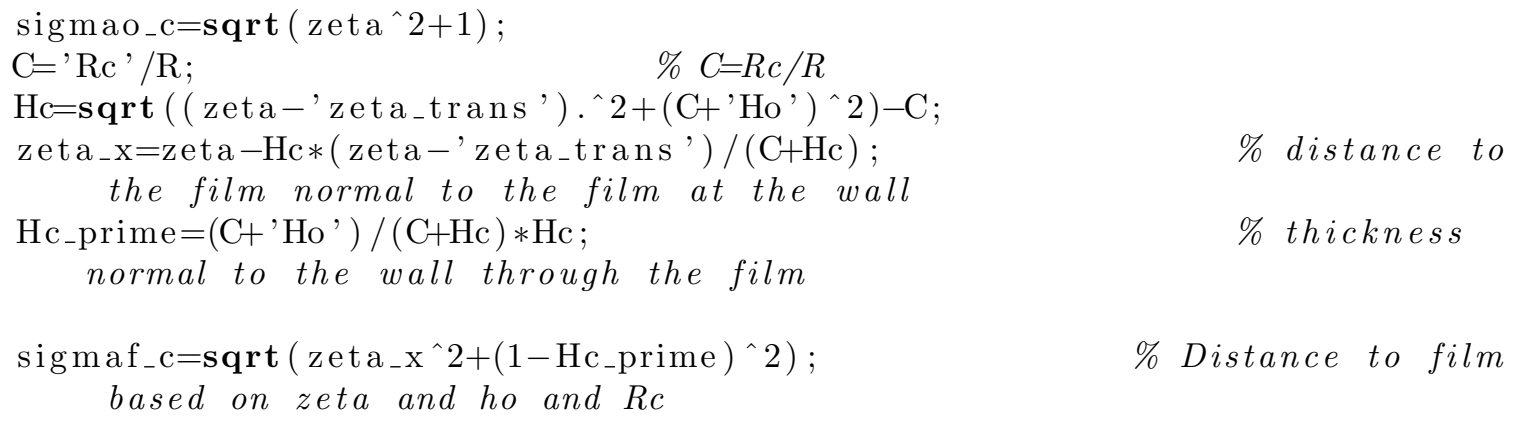


U_AB_c=subs ( subs (U_AB, sigmao, sigmao_c ), sigmaf, sigmaf_c );

Po\%\%

Corner Core B

\% For integrating across the film the perpedicular to the surface is used

$\operatorname{sigmao\_ c}=\mathbf{s q r t}($ zeta.^ $2+1)$;

$\mathrm{T}_{-} \mathrm{b}_{-} \mathrm{c}=-2 * \mathrm{U} \_\max * \mathrm{l}_{-} \mathrm{b}$;

for $\mathrm{i}=1$ : length $\left(\mathrm{Ho}_{-} \mathrm{O}\right)$;

$\mathrm{Ho}=\mathrm{Ho}_{-} \mathrm{O}(\mathrm{i})$;

$\mathrm{C}=\mathrm{Rc} . / \mathrm{R}_{-}$chan ; $\quad \% C=R c / R$

$\mathrm{Hc}=\mathbf{s q r t}\left((\right.$ zeta-zeta_trans $\left.(\mathrm{i})) \cdot{ }^{\wedge} 2+(\mathrm{C}+\mathrm{Ho}) \cdot{ }^{\wedge} 2\right)-\mathrm{C}$; C

$\mathrm{Hc}_{-}$prime $=(\mathrm{C}+\mathrm{Ho}) \cdot /(\mathrm{C}+\mathrm{Hc}) \cdot * \mathrm{Hc}$; normal to the wall through the film

$\%$ zet $a_{-} x=z$ et $a-H c . *\left(z e t a-z e t a_{-} \operatorname{trans}(i)\right) . /(C+H c)$; distance to the film normal to the film at the wall

$\% 0 \% \%$ ADDED -

$\%$ thickness

$\%$

zeta_x $=$ zeta-sqrt $\left(\mathrm{Hc}^{\wedge} 2-\mathrm{Hc}_{-}\right.$prime $\left.{ }^{\wedge} 2\right)$;

sigmaf_c $(\mathrm{i})=\operatorname{sigmao_{-}} \mathrm{c} *\left(1-\mathrm{Hc}_{-}\right.$prime $) \quad$;

\% Distance to film based on zeta and ho and $R c$

\%sigmaf-c $(i)=\operatorname{sqrt}\left(z \operatorname{eta} a_{-} x \cdot{ }^{\wedge} \mathscr{Z}+\left(1-H c_{-}\right.\right.$prime $\left.) .{ }^{\wedge} \mathscr{Q}\right)$;

$\%$ Distance

to film based on zeta and ho and $R c$

Fdrag_b_c_dzeta (i)=subs (subs (subs (subs (Fdrag_b_dz_dzeta, sigmao, sigmao_c) , sigmaf, sigmaf_c(i))/T_b_c), R, R_chan); \% subbed in sigmao sigmaf and $m u_{-} a$ and $m u_{-} b$

Fdrag_b_c_dzeta_string=char (vectorize (Fdrag_b_c_dzeta (i ) ) );

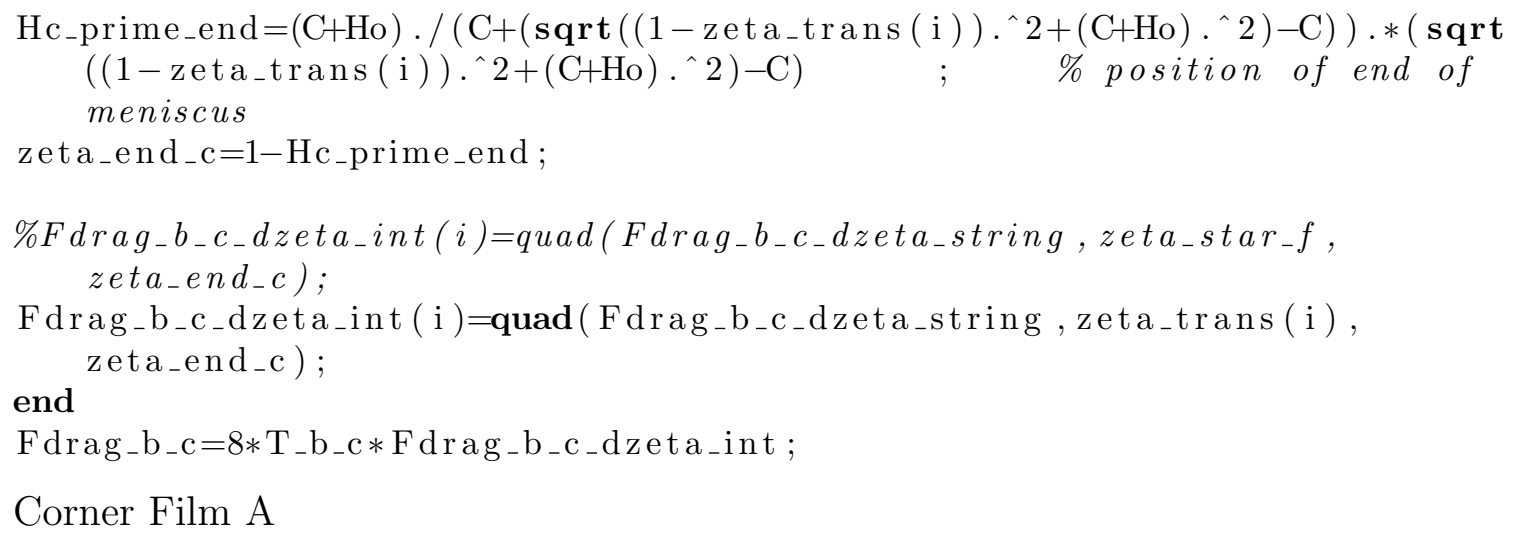

\% Film will use the same definitions for sigmaf and sigmao and mu_a and \% $m u_{-} b$ 
T_film_c $=-$ U_max*l_b ;

for $\mathrm{i}=1$ :length $\left(\mathrm{Ho}_{-} \mathrm{O}\right)$;

FIX sigma_f_c, current version uses sigma_x which is for the core

Fdrag_film_c_dzeta (i )=subs (subs (subs (subs (Fdrag_film_dz_dzeta, sigmao, sigmao_c), sigmaf, sigmaf_c(i))/ $\left.\mathrm{T}_{-}\left(\mathrm{film} \mathrm{m}_{-} \mathrm{c}\right), \mathrm{R}_{-} \mathrm{R}_{-} \mathrm{chan}\right) ; \%$ subbed in sigmao sigmaf and mu_a and mu_b

Fdrag_film_c_dzeta_string=char(vectorize (Fdrag_film_c_dzeta(i) ));

zeta_trans_c $(i)=z e t a_{-}$trans $(i)$;

Fdrag_film_c_dzeta_int ( i ) =quad( Fdrag_film_c_dzeta_string, zeta_trans_c (i)

end ,1) ;

Fdrag_film_c $=v p a(8 *$ Fdrag_film_c_dzeta_int*subs ( T_film_c ,R, R_chan $), 5)$;

Round

clear ho=1 syms tau_w_round_core mu_a mu_b Ho_o U_max U_ab l_b R_chan

for $i=1$ : length (ho)

U_ab (i ) =U_max./(1-mu_a $/ /(2 *$ mu_b $\left.) \cdot / \log \left(1-H_{o} \_o(i)\right)\right)$;

tau_f_round_core $(\mathrm{i})=-2 * \mathrm{mu}_{-} \mathrm{b} *\left(\mathrm{U}_{-} \max -\mathrm{U}_{-} \mathrm{ab}(\mathrm{i})\right) . /\left(\mathrm{R}_{-} \operatorname{chan} *\left(1-\mathrm{Ho}_{-} \mathrm{o}(\mathrm{i})\right)\right)$;

Fdrag_round_core $(\mathrm{i})=$ collect $\left(\right.$ tau_f_round_core $(\mathrm{i}) * \mathrm{l}_{-} \mathrm{b} * 2 * \mathbf{p} \mathbf{i} *\left(1-\mathrm{Ho}_{-} \mathrm{O}(\mathrm{i})\right)$

*R_chan, U_max) ; \%Tau_w*area of contact with the wall

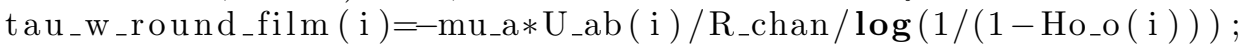

tau_f_round_film $(\mathrm{i})=-\mathrm{mu}_{-} \mathrm{a} * \mathrm{U}_{-} \mathrm{ab}(\mathrm{i}) /\left(\mathrm{R}_{-} \operatorname{chan} *\left(1-\mathrm{Ho}_{-} \mathrm{o}(\mathrm{i})\right)\right) / \log \left(1 /\left(1-\mathrm{Ho}_{-} \mathrm{O}\right.\right.$ (i) ));

Fdrag_round_film $(\mathrm{i})=\mathrm{tau}$ _w_round_film $_{-}(\mathrm{i}) * \mathrm{l}_{-} \mathrm{b} * 2 * \mathbf{p i} * \mathrm{R}_{-}$chan $;$

\%Tau_w*area of contact with the wall

end

Plotting Drag and ho

\% separate square with film

figure

hold on

plot (ho ( $3:$ length (ho ) ) , Fdrag_b_f_int $\left(3: \operatorname{length}\left(\mathrm{ho}_{-}\right)\right) * 2 * 8$, ho $(3: \operatorname{length}($ ho $))$, Fdrag_b_c_dzeta_int $(3:$ length $($ ho $)) * 2 * 8$, ho $(3:$ length $($ ho $))$,

Fdrag_film_f_int $(3:$ length $($ ho $)) * 8$, ho $(3:$ length $($ ho $))$,

Fdrag_film_c_dzeta_int $(3:$ length $($ ho $)) * 8)$;

plot (ho $(1: 2)$, Fdrag_b_f_int $(1: 2) * 2 * 8$, ho $(1: 2)$, Fdrag_b_c_dzeta_int $(1: 2)$ $* 2 * 8$, ho $(1: 2)$, Fdrag_film_f_int $(1: 2) * 8$, ho $(1: 2)$, Fdrag_film_c_dzeta_int $(1: 2) * 8)$;

hold off

$\%$ plot (ho, Fdrag_b_ $f_{-} i n t * 8 * R_{-}$chan)

$\%$ plot (ho, Fdrag-b_c_dzeta_int $* 8 * R_{-}$chan)

$\%$ plot (ho, Fdrag_film_f $f_{-} i n t * 8 * R_{-}$chan)

$\% \operatorname{plot}\left(h o, F d r a g_{-} f i l m_{-} c_{-} d z e t a_{-} i n t * 8 * R_{-} \operatorname{chan}\right)$ 


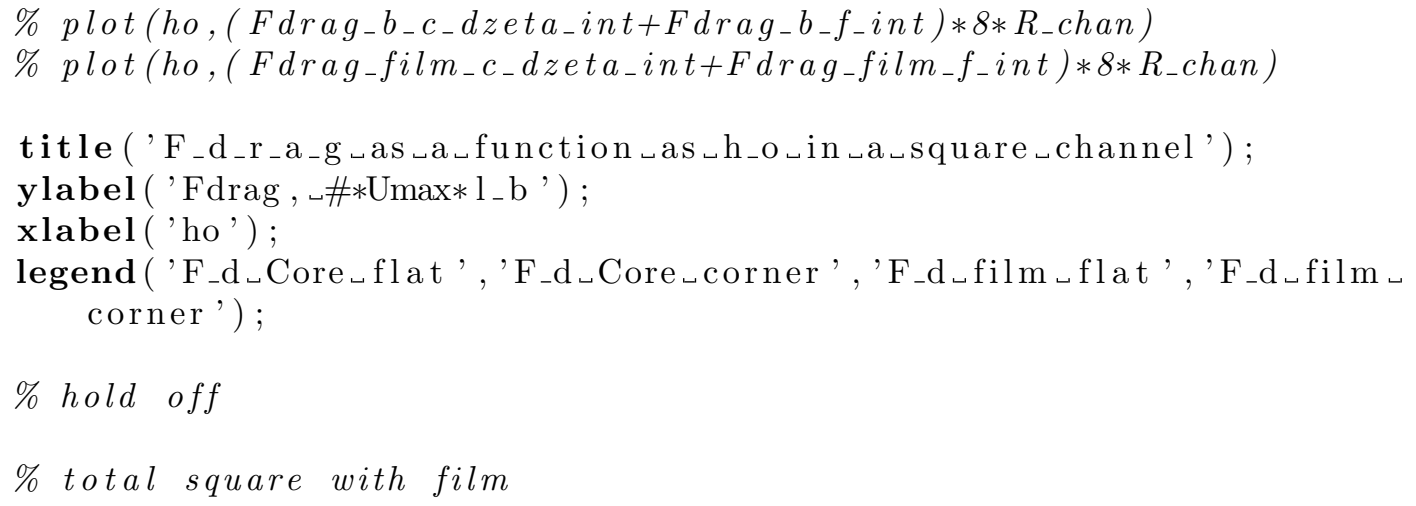

\section{figure}

hold on

plot (ho (3: length (ho)), (Fdrag _b_c_dzeta_int ( $3:$ length (ho ) ) +Fdrag_b_f_int $(3$ : length $($ ho $))) * 2 * 8$, ho ( 3 : length $($ ho $)),($ Fdrag_film_c_dzeta_int $(3$ : length(ho) ) +F drag_film_f_int ( 3 : length(ho) $)) * 8$, ho $(3:$ length (ho $)),(($ Fdrag_b_c_dzeta_int $(3:$ length (ho ) $)+$ Fdrag_b_f_int $(3:$ length (ho $))) * 2 * 8+($ Fdrag_film____dzeta_int ( $3:$ length $($ ho $))+$ Fdrag_film_f_int $(3:$ length (ho $)))$ $* 8))$;

plot (ho (1) , (Fdrag_b_c_dzeta_int (1)+Fdrag_b_f_int (1) ) $* 2 * 8$, 'o ', ho (1) , ( Fdrag_film_c_dzeta_int (1) +Fdrag_film_f_int (1) $) * 8$, 'o' , ho (1), ( ( Fdrag_b_c_dzeta_int (1)+Fdrag_b_f_int (1) $) * 2 * 8+($ Fdrag_film_c_dzeta_int $\left.\left.(1)+F d r a g \_f i l m \_f \_i n t(1)\right) * 8\right)$, 'o ', 'MarkerSize ',10, 'MarkerFaceColor' ,' green ', 'MarkerEdgeColor ', 'k' );

plot (ho (2), (Fdrag_b_c_dzeta_int (2)+Fdrag_b_f_int (2)) $* 2 * 8$, 'square', ho (2) , (Fdrag_film_c_dzeta_int (2)+Fdrag_film_f_int (2)) *8, 'square', ho (2), (( Fdrag_b_c_dzeta_int $\left.(2)+F d r a g_{-} b_{-} f_{-} i n t(2)\right) * 2 * 8+($ Fdrag_film_c_dzeta_int $\left.\left.(2)+F d r a g \_f i l m_{-} f_{-} i n t(2)\right) * 8\right)$, 'square ', 'MarkerSize ', 10 ,' MarkerFaceColor ', 'green ', 'MarkerEdgeColor ', 'k') ;

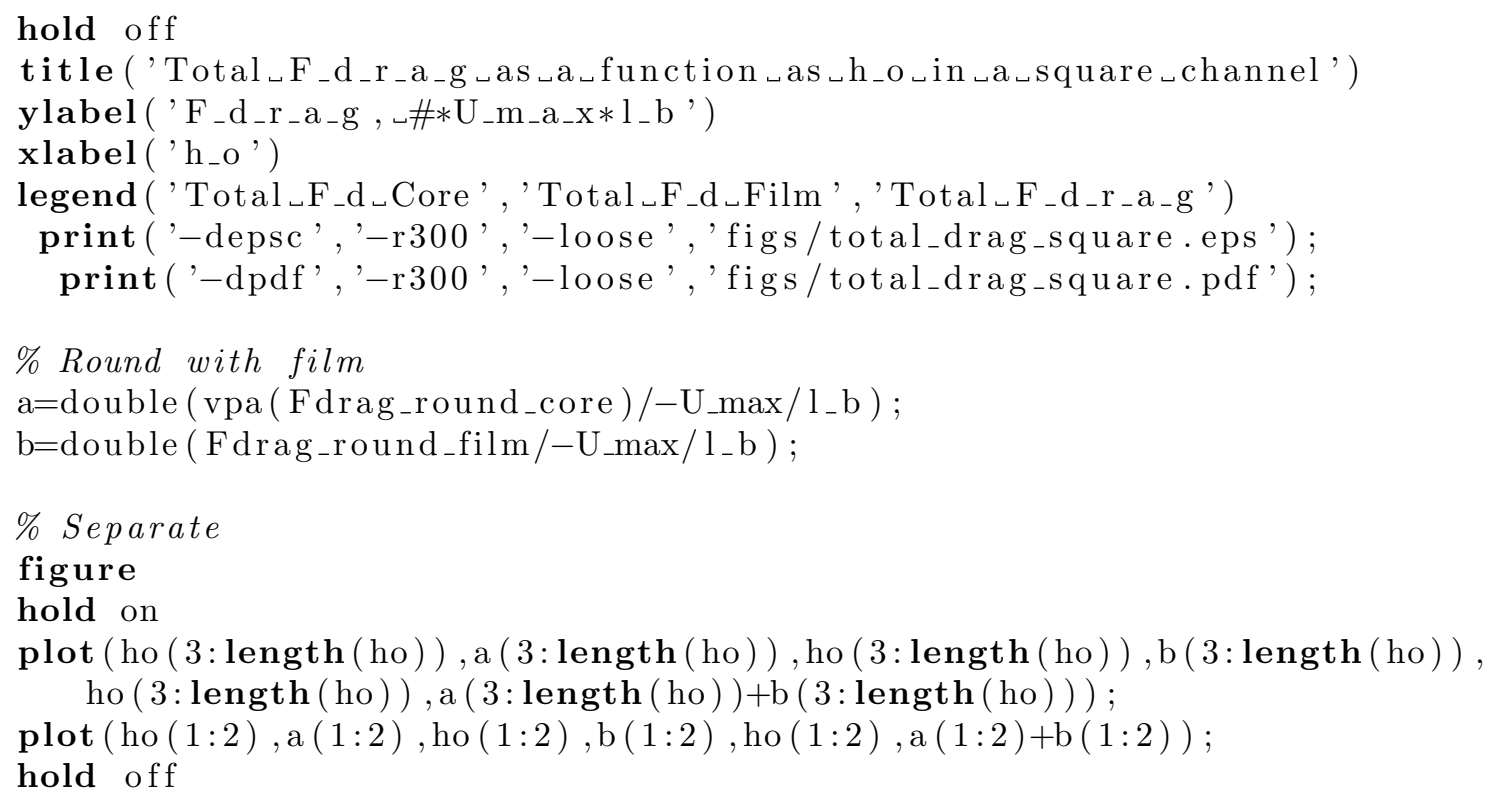




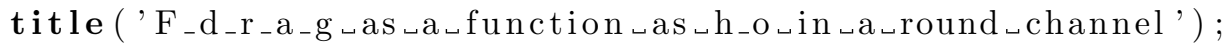

ylabel ('Fdrag, „\#\#*Umax*1_b');

xlabel ('ho');

legend ('F_d_Core ', 'F_d_film', 'Total_F_d') ;

print ('-depsc', '-r300', '-loose ', 'figs/total_drag_round.eps') ;

print ('-dpdf', '-r300', '-loose', ', figs/total_drag_round.pdf') ;

Table Production

\%table1=['ho',' Round F_drag Coefficient']

table $1=\left[\right.$ ho',$(\text { a }+ \text { b })^{\prime}$ ]

csvwrite('Round_drag_coefficient_ho_drag.csv', table1);

\%table2=['ho',' Square F_drag Coefficient']

table $2=$ [Ca_bound $(1: 2)$ ', ho $(1: 2)$ ',,$(($ Fdrag_b_c_dzeta_int $(1: 2)+$

Fdrag_b_f_int $(1: 2)) * 2 * 8+($ Fdrag_film_c_dzeta_int $(1: 2)+$

Fdrag_film_f_int $(1: 2)) * 8)^{\prime}$ '] ;

csvwrite(' square_drag_coefficient_ho_drag.csv', table2);

Force Balance

Stretch Term

clear mu_a

syms sigma_a sigma_ab C_B H_B sigma_b x_f H_A alpha R U_max l_a l_b beta mu_a

$\mathrm{F} \_$stretch $=2 * \mathbf{p i} * \mathrm{R} *\left(\left(1-\mathrm{H}_{-} \mathrm{A} / \mathrm{R}\right) *\left(\operatorname{sigma} \_\mathrm{b}+\operatorname{sigma} \_\mathrm{ab}-\mathrm{sigma} \mathrm{a}_{-}\right)-\mathrm{x}_{-} \mathrm{f} / \mathrm{R} *\left(\mathrm{sigma} \_\mathrm{b}+\right.\right.$

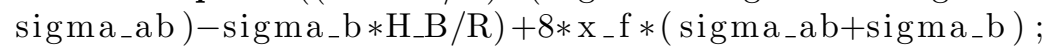

Pressure Term

$\operatorname{delP}=2 *\left(\operatorname{sigma} a_{-} a-\operatorname{sigma} a_{-} a b-\operatorname{sigma} a_{-} b\right) / R ;$

A_men=pi $* \mathrm{R}^{\wedge} 2$;

F_press $=$ delP $*$ A_men;

\section{Sum Terms}

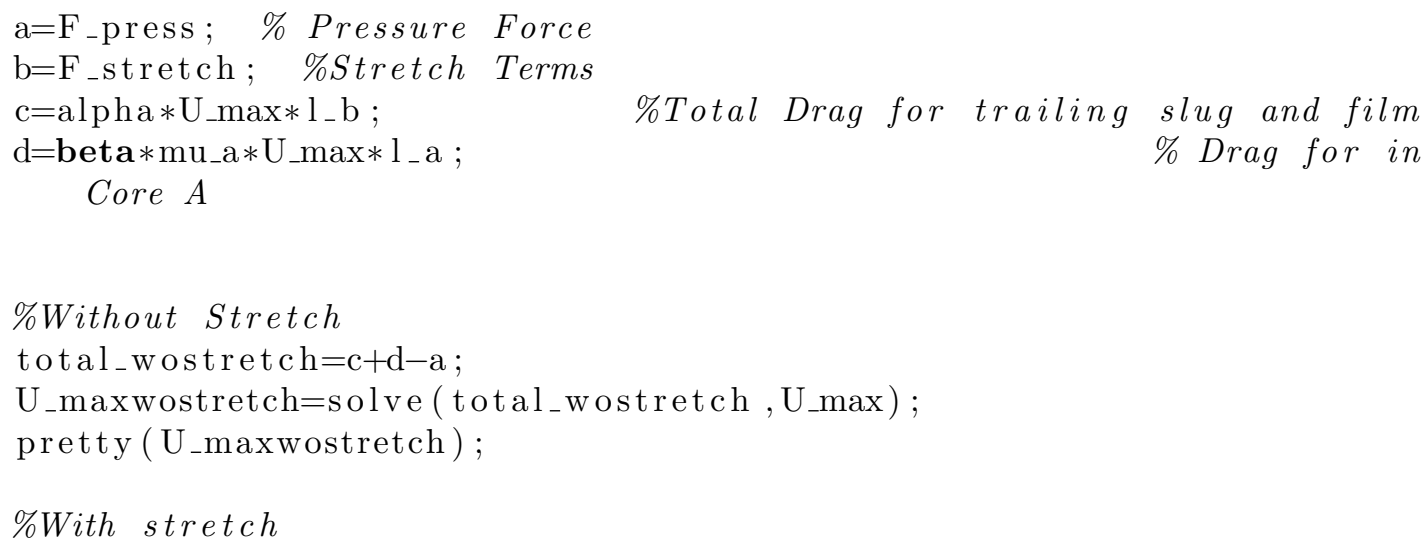




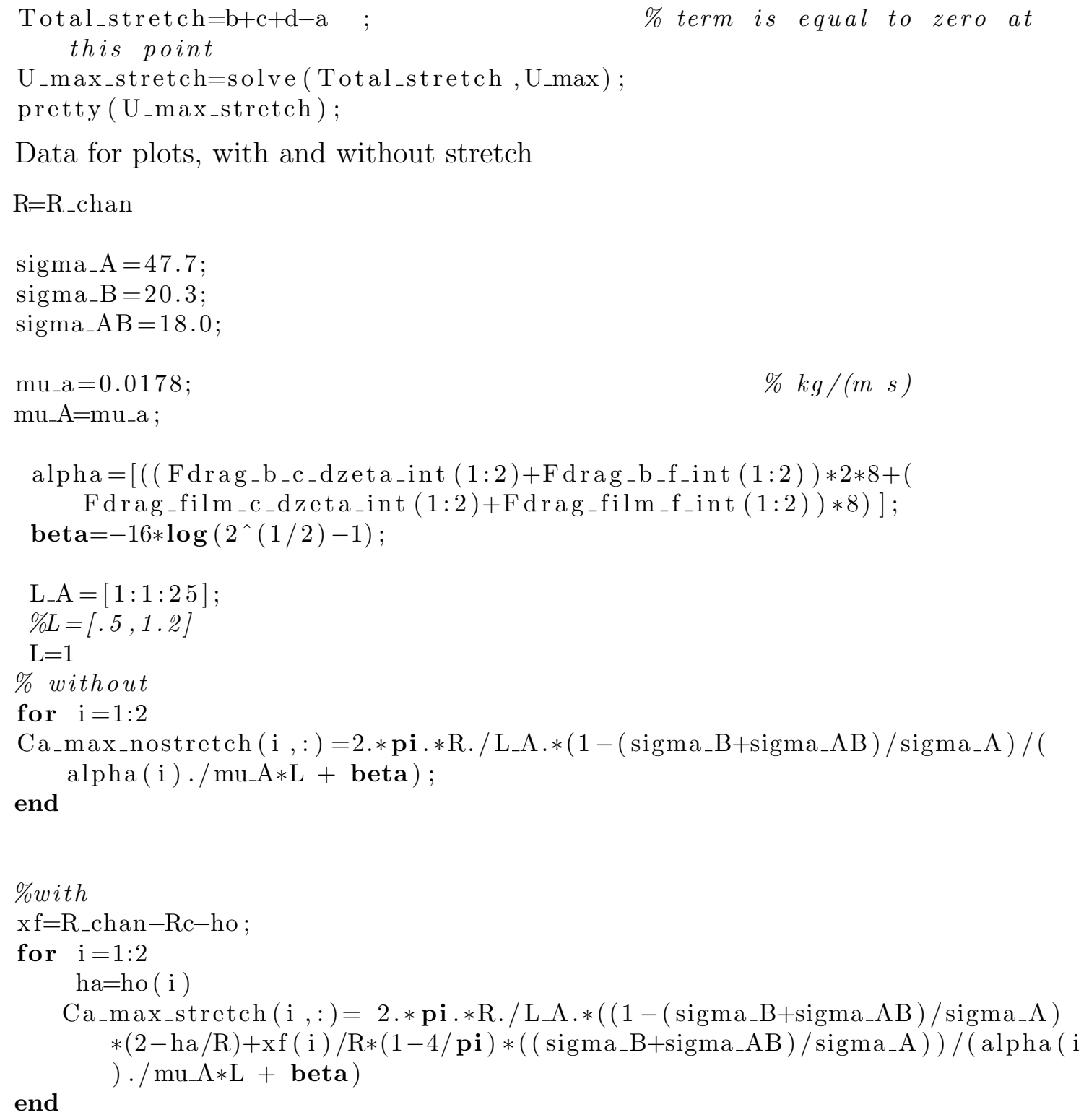


plot (L_A, Ca_max_stretch $(2,:)$, 'v' , 'MarkerEdgeColor ' , 'm') ;

plot (L_A_data, Ca_data_front, '+', ,'MarkerEdgeColor', ' red ') ;

hold off

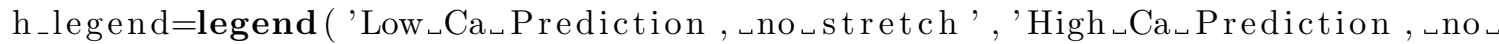

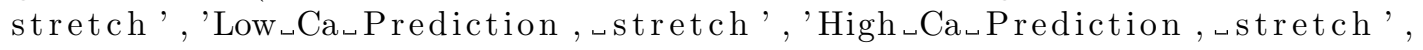

'Experimental_Data', ' Location ', 'SouthWest') ;

set (h_legend, 'FontSize', 8)

xlabel ('L_A $\mathrm{A}_{\sim}(\mathrm{mm})$ ')

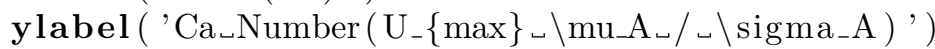

set (gca, 'XMinorTick', 'on ', 'Yscale', 'log', 'Xscale', 'log') ;

$x \lim ([.7,50])$;

$y \lim ([.00005, .05])$;

print ('-depsc', '-r300', ' figs/square_data_compare_front.eps ') ;

print ('-dpdf', '-r300', 'figs/square_data_compare_front.pdf')

Ca_data_rear=csvread ('10_19_0 $7_{\_}$square .csv', 1,6$)$;

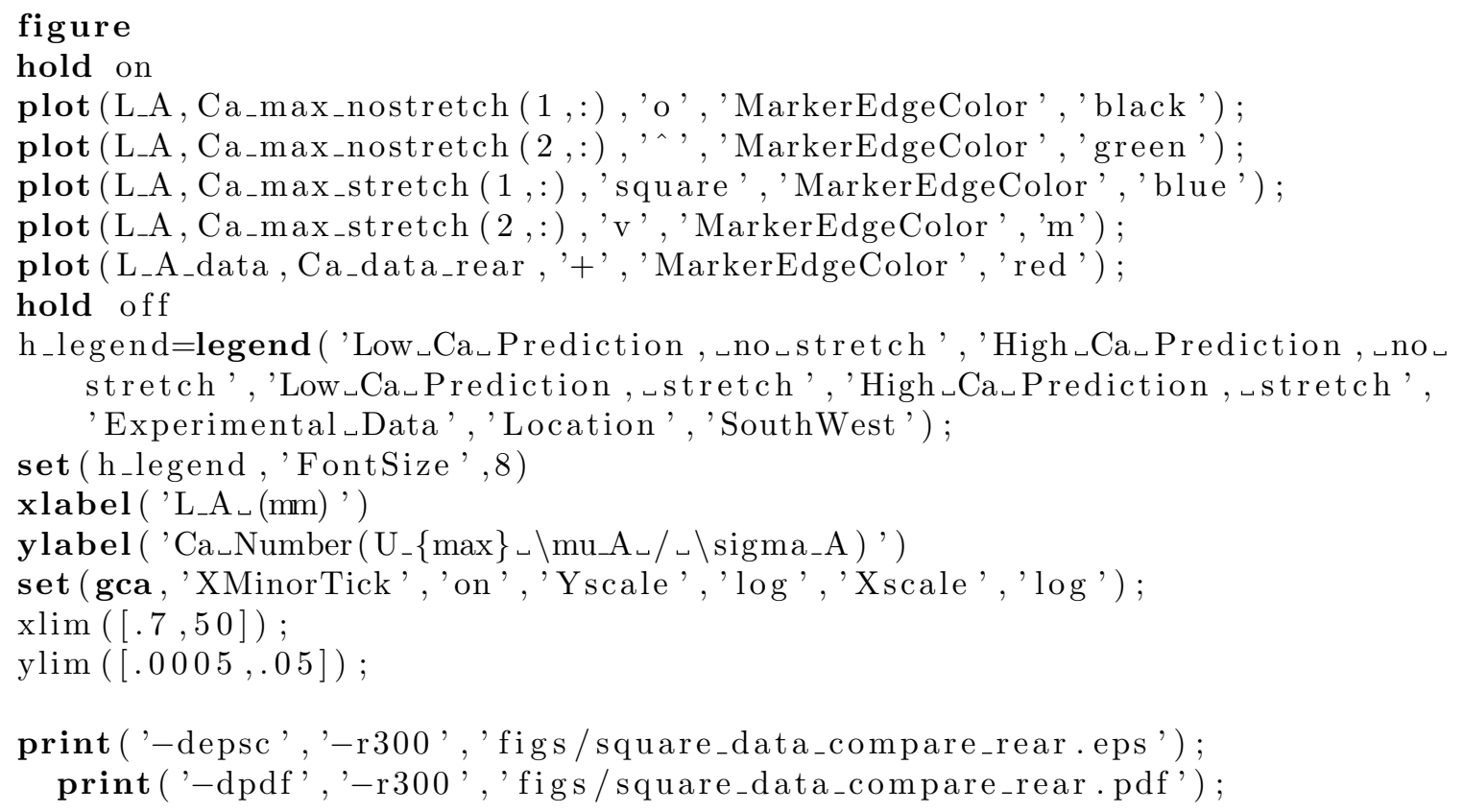

